\title{
Search for new particles in events with energetic jets and large missing transverse momentum in proton-proton collisions at $\sqrt{s}=13 \mathrm{TeV}$
}

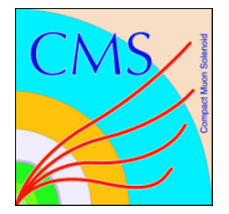

\section{The CMS collaboration}

E-mail: cms-publication-committee-chair@cern.ch

ABSTRACT: A search is presented for new particles produced at the LHC in proton-proton collisions at $\sqrt{s}=13 \mathrm{TeV}$, using events with energetic jets and large missing transverse momentum. The analysis is based on a data sample corresponding to an integrated luminosity of $101 \mathrm{fb}^{-1}$, collected in 2017-2018 with the CMS detector. Machine learning techniques are used to define separate categories for events with narrow jets from initial-state radiation and events with large-radius jets consistent with a hadronic decay of a $\mathrm{W}$ or $\mathrm{Z}$ boson. $\mathrm{A}$ statistical combination is made with an earlier search based on a data sample of $36 \mathrm{fb}^{-1}$, collected in 2016. No significant excess of events is observed with respect to the standard model background expectation determined from control samples in data. The results are interpreted in terms of limits on the branching fraction of an invisible decay of the Higgs boson, as well as constraints on simplified models of dark matter, on first-generation scalar leptoquarks decaying to quarks and neutrinos, and on models with large extra dimensions. Several of the new limits, specifically for spin-1 dark matter mediators, pseudoscalar mediators, colored mediators, and leptoquarks, are the most restrictive to date.

Keywords: Beyond Standard Model, Dark matter, Hadron-Hadron scattering (experiments), Higgs physics

ArXiv EPrint: 2107.13021 


\section{Contents}

1 Introduction 2

2 The CMS detector and event reconstruction 3

$\begin{array}{llr}3 & \text { Simulated samples } & 6\end{array}$

$\begin{array}{llr}4 & \text { Event selection } & 7\end{array}$

5 Background estimation $\quad 10$

$\begin{array}{ll}5.1 \text { Likelihood function } & 10\end{array}$

$\begin{array}{lll}5.2 & \text { Estimation of the QCD multijet background } & 11\end{array}$

$\begin{array}{lll}5.3 \text { Systematic uncertainties } & 14\end{array}$

6 Results and interpretation $\quad 16$

$\begin{array}{ll}\text { 6.1 Higgs portal interpretation } & 16\end{array}$

6.2 Interpretation in a DM simplified model with a colorless mediator $\quad 16$

$\begin{array}{ll}6.3 \text { Fermion portal interpretation } & 22\end{array}$

6.4 The ADD interpretation 22

6.5 Leptoquark interpretation 22

$\begin{array}{lll}7 & \text { Summary } & 24\end{array}$

A Additional figures and tables $\quad \mathbf{2 6}$

A.1 Event selection summary tables 26

$\begin{array}{ll}\text { A.2 Hadronic recoil distributions in the control regions } & 27\end{array}$

A.3 Exclusion in the Higgs portal interpretation split by data taking year 34

A.4 Coupling limits in a simplified DM model with a vector mediator 35

A.5 Two-dimensional exclusion in the simplified DM model with pseudoscalar $\begin{array}{ll}\text { mediator } & 36\end{array}$

A.6 Table of exclusion limits in the ADD model 37

$\begin{array}{ll}\text { B Supplemental material } & 38\end{array}$

B.1 Comparison with direct-detection experiments 38

$\begin{array}{lll}\text { B.2 Distributions of jet tagging variables } & 39\end{array}$

B.3 Large-radius jet tagging efficiencies for reinterpretation 40

B.4 Monojet $p_{\mathrm{T}}^{\text {miss }}$ distribution for the full data set 42

B.5 Analysis implementation in MADAnALYsis 43

B.6 Event display 45

The CMS collaboration $\quad 52$ 


\section{Introduction}

The standard model (SM) of particle physics has been widely recognized as a very successful, yet incomplete theory. Many important features of the universe, such as gravity and the existence of dark matter (DM), are not described in the SM. It is therefore paramount to search for evidence of physics beyond the SM (BSM). Attempts at finding BSM physics often center around the production of new, hypothetical particles, which subsequently decay to the observable SM particles. In this search, we aim at scenarios that are hidden from such searches, because the decay products of BSM particles are not necessarily detectable.

Scenarios with new particles that are not directly observable in collider detectors are motivated by many BSM theories. One of the strongest motivations stems from the idea of particle DM. Over the last decades, cosmological evidence for the existence of DM has been steadily accumulating [1], yet with few hints as to its nature or detailed properties. One theoretically attractive model of DM is that of a thermally produced weakly interacting massive particle (WIMP). If such a particle has just the right mass and couplings, the abundance of DM in the universe, as well as many of the observed phenomena commonly ascribed to DM, can be explained. In this search, multiple scenarios of DM production are considered. A Higgs portal scenario [2-4] is tested, in which DM particles are produced in decays of the Higgs boson [5-7]. Many of the properties of the new boson have already been measured with impressive precision, but a decay branching fraction $\mathcal{B}$ to nondetectable particles of up to about $20 \%$ is allowed by the current constraints [8, 9]. Beyond the Higgs portal scenario, simplified models of DM production [10] via new bosonic mediators with spin 0 or 1 are explored. Colorless mediators coupled to a pair of quarks and to a pair of DM particles are considered, as well as colored mediators, which decay into a single quark together with a single DM candidate. The latter scenario is referred to as a "fermion portal" $[11,12]$. In addition to a search for DM, a scenario with large extra dimensions proposed by Arkani-Hamed, Dimopoulos, and Dvali (ADD) [13, 14] is tested. In this model, the existence of additional spatial dimensions beyond the known three could explain the large difference in strength between the gravitational and electroweak (EW) interactions. In this scenario, gravitons can be produced in proton-proton (pp) collisions via their enhanced couplings to quarks or gluons and avoid detection by escaping in the additional dimensions. Representative Feynman diagrams for a subset of these signal models are shown in the first three panels of figure 1.

In these models the final-state particles are not detectable, but one needs a visible detector signature to be able to identify and record such events. We use energetic hadronic jets accompanying the invisible particles to select signal candidates. The experimental signature therefore comprises one or more energetic jets and large missing transverse momentum $\left(p_{\mathrm{T}}^{\text {miss }}\right)$. While the $p_{\mathrm{T}}^{\text {miss }}$ is the intrinsic result of BSM or SM particles escaping a detector without leaving any trace, hadronic jets derive from either initial-state gluon radiation or hadronic decays of energetic heavy SM vector bosons (V) produced in association with BSM particles. Production in association with a $\mathrm{V}$ boson is particularly important for the Higgs portal scenario, where the Higgs boson couples directly to the vector boson. For energetic V bosons, the hadronic decay products are Lorentz boosted in the laboratory 
frame and are reconstructed as a single large-radius jet with a characteristic substructure. Machine learning algorithms based on artificial neural networks are used in order to identify such signatures and efficiently suppress the overwhelming background coming from quantum chromodynamics (QCD) production of jets [15]. Separate signal categories are defined for events with and without an identified V candidate. Several control samples in data are used to constrain background contributions to the signal regions.

The chosen experimental signature can also be used to probe other BSM scenarios with new particles decaying into final states with visible and invisible particles. One such scenario probed by the present search is the production of leptoquarks (LQs). The LQs are hypothetical scalar or vector particles that carry both baryon and lepton numbers [16-18]. Here, a scenario with a single scalar LQ type is considered. This first-generation LQ decays into an up quark and an electron neutrino $\left(v_{\mathrm{e}}\right)$, and can be either produced in pairs [19] via a coupling to gluons, or singly $[20,21]$ in association with a $v_{\mathrm{e}}$, through its coupling to the up quark and $v_{\mathrm{e}}$. Both processes result in a jets $+p_{\mathrm{T}}^{\text {miss }}$ signature. A representative Feynman diagram for single LQ production is shown in the last panel of figure 1 .

Searches for new phenomena in events with jets and $p_{\mathrm{T}}^{\text {miss }}$ at $\sqrt{s}=13 \mathrm{TeV}$ have been previously published by the CMS [22] and ATLAS [23, 24] Collaborations. The search is carried out with the CMS detector at the CERN LHC, in pp collisions at $\sqrt{s}=13 \mathrm{TeV}$, using a data set collected in 2017-2018, corresponding to an integrated luminosity of $101 \mathrm{fb}^{-1}$. Compared to refs. [22], we have tripled the amount of analyzed data and enhanced the analysis sensitivity by means of improved identification of hadronically decaying $\mathrm{V}$ bosons. While such decays were previously selected using $N$-subjettiness [25], we now use a criteria based on a deep neural network. We have further extended the sensitivity by combining the new results with those from ref. [22], which are based on a data set of $36 \mathrm{fb}^{-1}$, yielding a total data set of $137 \mathrm{fb}^{-1}$, equivalent in size to that of ref. [24].

This paper is organized as follows. After discussing the CMS detector in section 2 and the simulated samples in section 3 , we describe the event selection in section 4 , followed by the background estimation in section 5 . Section 6 contains the results of the analysis and their interpretation in the context of the above scenarios. We summarize the paper in section 7. Tabulated results, as well as extensive material for use in reinterpretation, are provided in HEPData [26]. To further aid reinterpretation, an implementation of the analysis selection is provided in the MADANALYsis framework [27-29]. Information related to the validation of this implementation is provided as supplementary material.

\section{The CMS detector and event reconstruction}

The central feature of the CMS apparatus is a superconducting solenoid of $6 \mathrm{~m}$ internal diameter, providing a magnetic field of $3.8 \mathrm{~T}$. Within the solenoid volume are a silicon pixel and strip tracker, a lead tungstate crystal electromagnetic calorimeter (ECAL), and a brass and scintillator hadron calorimeter (HCAL), each composed of a barrel and two endcap sections. Forward calorimeters extend the pseudorapidity $(\eta)$ coverage provided by the barrel and endcap detectors. Muons are detected in gas-ionization detectors embedded in the steel flux-return yoke outside the solenoid. 

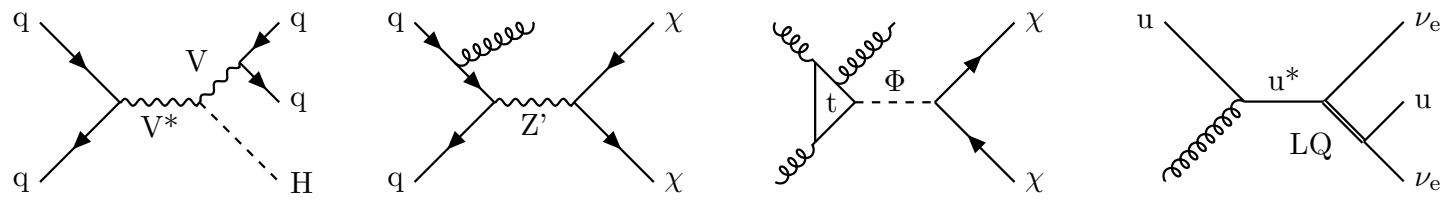

Figure 1. Representative Feynman diagrams for a number of signal models: Higgs production in association with an SM vector boson (left), colorless spin-1 and spin-0 mediators (middle left and right, respectively), single leptoquark production (right). In all cases, subdominant production modes not pictured here are taken into account, as described in the text.

The silicon tracker measures charged particles within the pseudorapidity range $|\eta|<$ 2.5. During the LHC running period when the data used in this paper were recorded, the silicon tracker consisted of 1856 silicon pixel and 15148 silicon strip detector modules.

In the region $|\eta|<1.74$, the HCAL cells have widths of 0.087 in pseudorapidity and 0.087 in azimuth $(\phi)$. In the $\eta-\phi$ plane, and for $|\eta|<1.48$, the HCAL cells map on to $5 \times 5$ arrays of ECAL crystals to form calorimeter towers projecting radially outwards from close to the nominal interaction point. For $|\eta|>1.74$, the coverage of the towers increases progressively to a maximum of 0.174 in $\Delta \eta$ and $\Delta \phi$. The hadron forward (HF) calorimeter uses steel as an absorber and quartz fibers as the sensitive material. The two halves of the $\mathrm{HF}$ are located $11.2 \mathrm{~m}$ from the interaction region, one on each end, and together they provide coverage in the range $3.0<|\eta|<5.2$.

Events of interest are selected using a two-tiered trigger system. The first level (L1), composed of custom hardware processors, uses information from the calorimeters and muon detectors to select events at a rate of around $100 \mathrm{kHz}$ within a fixed latency of about $4 \mu \mathrm{s}$ [30]. The second level, known as the high-level trigger (HLT), consists of a farm of processors running a version of the full event reconstruction software optimized for fast processing, and reduces the event rate to around $1 \mathrm{kHz}$ before data storage [31].

A more detailed description of the CMS detector, together with a definition of the coordinate system used and the relevant kinematic variables, can be found in ref. [32].

The candidate vertex with the largest value of summed physics-object transverse momenta $p_{\mathrm{T}}^{2}$ is taken to be the primary vertex $(\mathrm{PV})$ of the pp interaction. The physics objects are the jets, clustered using the jet finding algorithm [33, 34] with the tracks assigned to candidate vertices as inputs, and the associated missing transverse momentum, taken as the negative vector sum of the $p_{\mathrm{T}}$ of those jets.

A particle-flow (PF) algorithm [35] aims to reconstruct and identify each individual particle in an event, with an optimized combination of information from the various elements of the CMS detector. In this process, the identification of the PF candidate type (photon, electron, muon, and charged and neutral hadrons) plays an important role in the determination of the particle direction and energy. The energy of photons is obtained from the ECAL measurement. The energy of electrons is determined from a combination of the electron momentum at the PV as determined by the tracker, the energy of the corresponding ECAL cluster, and the energy sum of all bremsstrahlung photons spatially compatible 
with originating from the electron track. The energy of muons is obtained from the curvature of the corresponding track. The energy of charged hadrons is determined from a combination of their momentum measured in the tracker and the matching ECAL and HCAL energy deposits, corrected for the response function of the calorimeters to hadronic showers. Finally, the energy of neutral hadrons is obtained from the corresponding corrected ECAL and HCAL energies.

For each event, hadronic jets are clustered from the PF candidates using the infraredand collinear-safe anti- $k_{\mathrm{T}}$ algorithm $[33,34]$ with a distance parameter of 0.4 or 0.8 . Depending on the respective distance parameter, these jets are referred to as "AK4" or "AK8" jets. Jet momentum is determined as the vectorial sum of all particle momenta in the jet, and is found from simulation to be, on average, within 5 to $10 \%$ of the true momentum over the entire $p_{\mathrm{T}}$ spectrum and detector acceptance [36]. Additional pp interactions within the same or nearby bunch crossings (pileup) can contribute additional tracks and calorimetric energy depositions to the jet momentum. To mitigate this effect, charged particles identified as not originating from the PV are discarded and an offset correction is applied to correct for the remaining neutral pileup contributions [36]. Jet energy corrections are derived from simulation to bring the measured response of jets to that of particle-level jets on average. In situ measurements of the momentum balance in the dijet, $\gamma+$ jet, $\mathrm{Z}+$ jet, and multijet events are used to account for any residual differences in the jet energy scale (JES) and jet energy resolution (JER) in data and simulation [36]. The jet energy resolution amounts typically to $15-20 \%$ at $30 \mathrm{GeV}, 10 \%$ at $100 \mathrm{GeV}$, and $5 \%$ at $1 \mathrm{TeV}$ [36]. Additional selection criteria [37] are applied to each jet to remove jets potentially dominated by anomalous contributions from various subdetector components or reconstruction failures.

The missing transverse momentum vector $\vec{p}_{\mathrm{T}}^{\text {miss }}$ is computed as the negative vector sum of the transverse momenta of all the PF candidates in an event, and its magnitude is denoted as $p_{\mathrm{T}}^{\text {miss }}$. The $\vec{p}_{\mathrm{T}}^{\text {miss }}$ is modified to account for corrections to the energy scale and resolution of the reconstructed jets in the event [38]. Anomalous high- $p_{\mathrm{T}}^{\text {miss }}$ events can be due to a variety of reconstruction failures, detector malfunctions, or noncollision backgrounds. Such events are rejected by dedicated filters that are designed to eliminate more than $85-90 \%$ of the spurious high- $p_{\mathrm{T}}^{\text {miss }}$ events with a signal efficiency exceeding $99.9 \%$ [38].

Large-radius AK8 jets are used for the identification of hadronic decays of $\mathrm{W}$ and $\mathrm{Z}$ bosons. The pileup-per-particle identification (PUPPI) algorithm [39] is used to mitigate the effect of pileup at the reconstructed-particle level, making use of local shape information, event pileup properties, and tracking information. Charged particles identified as not originating from the PV are discarded. For each neutral particle, a local shape variable is computed using the surrounding charged particles within the tracker acceptance $(|\eta|<2.5)$ compatible with the PV, and using both charged and neutral particles in the region outside of the tracker coverage. The momenta of the neutral particles are then rescaled according to their probability to originate from the PV deduced from the local shape variable, avoiding the need for jet-based pileup corrections [37]. The modified mass drop tagger algorithm [40, 41], also known as the soft-drop (SD) algorithm, with the angular exponent $\beta=0$, soft cutoff threshold $z_{\text {cut }}<0.1$, and characteristic radius $R_{0}=0.8$ [42], is applied to remove soft, wide-angle radiation from the jet. 


\section{Simulated samples}

Monte Carlo (MC) simulated event samples are used to model signal and background contributions to all the analysis regions. In all cases, parton showering, hadronization, and underlying event properties are modeled using PYTHIA [43] version 8.202 or later with the underlying event tune CP5 [44]. Simulation of interactions between particles and the CMS detector is based on GEant4 [45]. The same reconstruction algorithms used for data are applied to simulated samples. The NNPDF3.1 next-to-next-to-leading order (NNLO) set of parton distribution functions (PDFs) [46] is used for the generation of all samples.

For the $\mathrm{V}+$ jets processes, predictions with up to two partons in the final state are obtained at next-to-leading order (NLO) in QCD using MADGRAPH5_aMC@NLO version 2.4.2 [47] with the FxFx matching scheme [48] between the jets from the matrix element calculations and the parton shower. The $\gamma+$ jets samples are simulated at NLO in QCD with up to one additional parton using MADGRAPH5_aMC@NLO version 2.6.5. This version is also used for all other MADGRAPH5_aMC@NLO samples, unless indicated otherwise. Samples of events with top quark pairs are generated at NLO in QCD with up to two additional partons in the matrix element calculations using MADGRAPH5_aMC@NLO and the FxFx jet matching scheme. Their cross sections are normalized to the inclusive cross section of the top quark pair production at NNLO in QCD [49]. Events with single top quarks are simulated using POWHEG $2.0[50,51]$ and normalized to the inclusive cross section calculated at NNLO in QCD [52] for single top quarks produced in association with a $\mathrm{W}$ boson, and NLO in QCD [53, 54] for production in association with a quark. Production of diboson events (WW, WZ, and ZZ) is simulated at leading order (LO) in QCD using PYTHIA, and normalized to the cross sections at NNLO precision for WW production [55] and at NLO precision for the others [56]. The production of $\mathrm{W} \gamma$ and $\mathrm{Z} \gamma$ events is simulated using MADGRAPH5_amC@NLO at NLO in QCD. Samples of QCD multijet events are generated at LO using PYTHIA.

For the Higgs portal signal model, POWHEG is used to generate separate signal samples for the different production modes of the Higgs boson: via gluon fusion [57], in association with a SM vector boson (VH) [58], and via vector boson fusion (VBF) [59]. The samples are generated by enforcing decays of the SM Higgs boson to neutrinos, and are normalized to the SM cross sections evaluated at next-to-NNLO in QCD and NLO in EW corrections for the gluon fusion production, and at NNLO in QCD and NLO in EW for the VBF and VH modes [60]. Events for the simplified model scenarios of DM production are generated using MAdGraph5_amC@NLO and the DMsimp model implementation [61-63]. For the case of spin-1 mediators, events with a pair of DM particles and either one or two additional partons are generated at NLO in QCD, and the FxFx jet matching is used. The couplings between the mediator and quarks, as well as between the mediator and the DM particles, are set to $g_{\mathrm{q}}=0.25$ and $g_{\chi}=1.0$, respectively, as recommended by the LHC Dark Matter Working Group [64]. For DM production via spin-0 mediators, which is loop-induced, signal samples are generated at $\mathrm{LO}$ with one additional parton in the matrix element calculations, and the respective couplings are set to $g_{\mathrm{q}}=g_{\chi}=1.0$ [64]. Separate samples are generated for different coupling types (vector, axial vector, scalar, and pseudoscalar), as well as 
for different mass hypotheses for the mediator and DM particles. Signal events for the fermion portal scenario are generated using MADGRAPH5_aMC@NLO and the S3D_uR implementation of ref. [65]. In this case, the mediator is assumed to couple to right-handed up quarks and a Dirac fermion DM candidate with a coupling of $\lambda_{\mathrm{FP}}=1$. The single and pair production of scalar LQs are simulated at LO in QCD using MADGRAPH5_aMC@NLO version 2.6.0 with an implementation provided by the authors of ref. [19]. Decays of each LQ to an up quark and an electron neutrino are enforced, and separate samples are generated for the LQ mass values between 0.5 and $2.5 \mathrm{TeV}$, as well as for the LQ-u- $\mathrm{v}_{\mathrm{e}}$ coupling values $\lambda_{\mathrm{LQ}}$ ranging from 0.01 to 1.5 , depending on the LQ mass. Finally, events with graviton production in the ADD scenario are generated at LO using PYTHIA [66]. In this case, samples of signal events are generated for the number of extra dimensions $d$ between 2 and 7 , and the values of the fundamental Planck scale $M_{\mathrm{D}}$ between 5 and $15 \mathrm{TeV}$.

\section{Event selection}

The key feature of the analysis is the extensive use of control data samples for the purpose of precise prediction of the background contributions in the signal regions (SRs), which contain events with high- $p_{\mathrm{T}}$ jets and large $p_{\mathrm{T}}^{\text {miss }}$. The leading SM background contributions originate from $\mathrm{Z} \rightarrow \mathrm{v} v$ and $\mathrm{W} \rightarrow \ell v$ production $(\ell=\mathrm{e}, \mu, \tau)$, the properties of which are constrained using control regions (CRs) with charged leptons that are enriched in $\mathrm{Z} \rightarrow \ell \ell$ and $\mathrm{W} \rightarrow \ell v$ events, respectively. Additionally, CRs enriched in $\gamma+$ jets events are defined. The $\mathrm{V}+$ jets events in these CRs share many kinematic properties of the processes in the SRs and are used to constrain the latter. The CR and SR definitions share as many of the selection criteria as possible, in order to ensure that minimal selection biases are introduced. For each SR, five CRs are defined: dielectron and dimuon CRs enriched in $\mathrm{Z} \rightarrow \ell \ell$ events, single-electron and single-muon CRs enriched in $\mathrm{W} \rightarrow \ell v$ events, and a fifth CR enriched in $\gamma+$ jets events.

The SR events are selected using a trigger with a $p_{\mathrm{T}}^{\text {miss }}$ requirement of at least $120 \mathrm{GeV}$. The trigger requirement for the SRs is based on an online calculation of $p_{\mathrm{T}}^{\text {miss }}$ based on all PF candidates reconstructed at the HLT, except for muons. Events with high- $p_{\mathrm{T}}$ muons are therefore also assigned large online $p_{\mathrm{T}}^{\text {miss }}$, and the same trigger is used to collect data populating the single-muon and dimuon CRs. The control samples with electrons are selected based on two different single-electron triggers requiring of $p_{\mathrm{T}}>35(32) \mathrm{GeV}$ for $2017(2018)$ and $p_{\mathrm{T}}>115 \mathrm{GeV}$, and on a single-photon trigger with a requirement of $p_{\mathrm{T}}>200 \mathrm{GeV}$. The single-electron triggers differ in their usage of isolation requirements: while the lower threshold trigger requires electrons to be well isolated, the higher-threshold trigger does not, which gives an improved efficiency at high $p_{\mathrm{T}}$. Similarly, the singlephoton trigger avoids the reliance on the online track reconstruction and increases the overall efficiency for electrons with $p_{\mathrm{T}}>200 \mathrm{GeV}$. The photon trigger is also used to select events for the photon control samples. During the 2017 data taking, a gradual shift in the timing of the inputs of the ECAL L1 trigger in the region at $|\eta|>2.0$ caused a specific trigger inefficiency. For events containing an electron or a photon (a jet) with $p_{\mathrm{T}} \gtrsim 50$ (100) $\mathrm{GeV}$ in this region, the efficiency loss is up to $\approx 10-20 \%$, depending on $p_{\mathrm{T}}, \eta$, and 
time. Correction factors are computed from data and applied to the acceptance evaluated by simulation for the 2017 samples.

At the analysis level, a requirement of $p_{\mathrm{T}}^{\text {miss }}>250 \mathrm{GeV}$ is applied to the SR events in order to ensure a $p_{\mathrm{T}}^{\text {miss }}$ trigger efficiency of at least $95 \%$. Events are separated into three mutually exclusive categories based on the properties of the highest $p_{\mathrm{T}}$ ("leading") jet in the event: low-purity mono- $\mathrm{V}$, high-purity mono- $\mathrm{V}$, and monojet. For the mono- $\mathrm{V}$ categories, the leading AK8 jet is required to have $p_{\mathrm{T}}>250 \mathrm{GeV}$ and $|\eta|<2.4$. In order to preferentially select events where an AK8 jet originates from a hadronic decay of a $\mathrm{W}$ or $\mathrm{Z}$ boson, the jet is further required to be $\mathrm{V}$ tagged with the DEEPAK8 algorithm [15] and to have an SD-corrected mass of $65<m_{\mathrm{SD}}<120 \mathrm{GeV}$. The DeEPAK 8 algorithm employs a deep neural network to differentiate between jets from vector boson, top quark, and Higgs boson decays, as well as jets originating from QCD radiation. The inputs to the neural network are features of up to 100 jet constituent PF candidates of a given jet and features related to up to seven secondary vertices reconstructed in a given collision event. For each jet, the output of the neural network is one numerical score for each of the jet classes, representing the likelihood that the jet originates from that class. In this analysis, separation between vector boson and QCD jets is sought, and a binary score is constructed by taking the ratio of the vector boson score to the sum of vector boson and QCD scores. The assignment to low- and high-purity mono- $\mathrm{V}$ categories is then based on the binary score of the leading jet. The high-purity category selects genuine $\mathrm{V}$ jets (QCD jets) with an efficiency of $30(0.7) \%$ at a jet $p_{\mathrm{T}}$ of $250 \mathrm{GeV}$, rising to $40(0.7) \%$ at $800 \mathrm{GeV}$. For jets failing the high-purity selection, the low-purity selection has an efficiency of $40(7) \%$ at $250 \mathrm{GeV}$, falling to 30 (5)\% at $800 \mathrm{GeV}$. Compared to the $N$-subjettiness-based selection employed in the previous analysis [22], the DEEPAK8 tagger reduces the rate of QCD jets incorrectly identified as vector boson jets by a factor of five to ten depending on jet $p_{\mathrm{T}}$ without reducing the efficiency for genuine $\mathrm{V}$ jets. Events that do not pass the mono- $\mathrm{V}$ selection are considered for the monojet category. In this case, the leading AK4 jet in the event is required to have $p_{\mathrm{T}}>100 \mathrm{GeV},|\eta|<2.4$, and to pass quality criteria based on the composition of the jet in terms of different types of PF candidates, such as a minimum charged-hadron energy fraction of $10 \%$ and a maximum neutral-hadron energy fraction of $80 \%$ [37].

In all categories, further requirements are imposed in order to suppress reducible background processes. Events are rejected if they contain a well-reconstructed and isolated electron (photon) with $p_{\mathrm{T}}>10(15) \mathrm{GeV}$ and $|\eta|<2.5$, or a muon with $p_{\mathrm{T}}>10 \mathrm{GeV}$ and $|\eta|<2.4$ [67, 68]. Hadronically decaying $\tau$ leptons are identified using the "hadronsplus-strips" algorithm and a multivariate classifier at a working point corresponding to an efficiency of $70 \%$ for genuine $\tau$ decays and $0.5-3 \%$ for jets from QCD production, depending on jet $p_{\mathrm{T}}$ [69]. Events with a hadronically decaying $\tau$ lepton candidate with $p_{\mathrm{T}}>18 \mathrm{GeV}$ and $|\eta|<2.3$ are removed. These requirements efficiently reject events with leptonic decays of the $\mathrm{V}$ bosons and top quarks, as well as backgrounds with photons. Contributions from top quark processes are further suppressed by rejecting events with AK4 jets that have $p_{\mathrm{T}}>20 \mathrm{GeV},|\eta|<2.4$, and are identified to have originated from the hadronization of a bottom quark ("b-tagged jets") using the DEEPCSV algorithm with a "medium" working point, corresponding to correctly identifying a b jet with a probability 
of $80 \%$ and misidentifying a light-flavor quark or gluon jet with a probability of $10 \%$ [70]. Finally, topological requirements are applied in order to reject contributions from QCD multijet events. These events do not have $p_{\mathrm{T}}^{\text {miss }}$ from genuine sources and require a $p_{\mathrm{T}}^{\text {miss }}$ mismeasurement in order to pass the SR selections, which can happen in two main ways. In the first case, the energy of a jet in the event could be misreconstructed either as a result of an interaction between the jet with poorly instrumented or inactive parts of the detector, or because of failures in the readout of otherwise functioning detector modules. In these cases, artificial $p_{\mathrm{T}}^{\text {miss }}$ is generated with a characteristically small azimuthal angle difference between the misreconstructed jet $\vec{p}_{\mathrm{T}}$ and the $\vec{p}_{\mathrm{T}}^{\text {miss }}$ vectors. Such events are rejected by requiring $\Delta \phi\left(\vec{p}_{\mathrm{T}}^{\text {jet }}, \vec{p}_{\mathrm{T}}^{\text {miss }}\right)>0.5$. In the second case, large $p_{\mathrm{T}}^{\text {miss }}$ is generated due to failures of the $\mathrm{PF}$ reconstruction, which are suppressed by considering an alternative calculation of $p_{\mathrm{T}}^{\text {miss }}$ based on calorimeter energy clusters and muon candidates, rather than the full set of all $\mathrm{PF}$ candidates. While the calorimeter-based $p_{\mathrm{T}}^{\text {miss }}$ has significantly worse resolution than $\mathrm{PF} p_{\mathrm{T}}^{\mathrm{miss}}$, it is much simpler and more robust. To reduce the multijet background caused by $\mathrm{PF}$ reconstruction failures, events are required to have $\Delta p_{\mathrm{T}}^{\text {miss }}(\mathrm{PF}$-calorimeter $)=\mid p_{\mathrm{T}}^{\text {miss }}(\mathrm{PF}) / p_{\mathrm{T}}^{\text {miss }}($ calorimeter $)-1 \mid<0.5$. A similar criterion is constructed using an alternative $p_{\mathrm{T}}^{\text {miss }}$ calculation based exclusively on charged-particle candidates. Since charged particles are only reconstructed within the coverage of the pixel tracking detector, this $p_{\mathrm{T}}^{\text {miss }}$ variant is robust against noise and $\mathrm{PU}$ contributions in the forward calorimeters. Events in the SR are required to have a maximum angular separation in the transverse plane between the regular and charged-particle candidate $p_{\mathrm{T}}^{\text {miss }}$ vectors of $\Delta \phi(\mathrm{PF}$, charged $)<2$. Finally, a section of the HCAL was not functioning during a part of the 2018 data taking period corresponding to $65 \%$ of the total integrated luminosity recorded in that year, leading to irrecoverable mismeasurement in a localized region of the detector $(-1.57<\phi<-0.87,-3.0<\eta<-1.3)$. To avoid contamination from such mismeasurement, events where any jet with $p_{\mathrm{T}}>30 \mathrm{GeV}$ is found in the corresponding $\eta$ - $\phi$ region are rejected in the analysis of the 2018 data set. Events where the mismeasurement is so severe that a jet is fully lost in this region are found to contribute at low values of $p_{\mathrm{T}}^{\text {miss }}<470 \mathrm{GeV}$ and to have a characteristic signature in $\phi\left(\vec{p}_{\mathrm{T}}^{\text {miss }}\right)$. Such events are rejected by requiring that $\phi\left(\vec{p}_{\mathrm{T}}^{\text {miss }}\right) \notin[-1.62,-0.62]$ if $p_{\mathrm{T}}^{\text {miss }}<470 \mathrm{GeV}$. The value of $470 \mathrm{GeV}$ is the boundary of the optimal signal region binning just above this contamination region.

In each of the CRs, the same selection criteria are applied as for the corresponding SR (monojet, or low- or high-purity mono-V), with two exceptions: the charged-lepton and photon rejection criteria are inverted to allow the exact number of desired leptons or photons for each $\mathrm{CR}$, and the $\vec{p}_{\mathrm{T}}^{\text {miss }}$ vector used in the SR definition is replaced by the hadronic recoil vector $\vec{U}$. The hadronic recoil is defined as the vectorial sum of the $\vec{p}_{\mathrm{T}}^{\text {miss }}$ vector and the transverse momentum vectors of the selected charged lepton(s) or the photon in each event. The hadronic recoil therefore acts as a proxy of the momentum of the $\mathrm{V}$ boson or a photon in each CR, convolved with the $p_{\mathrm{T}}^{\text {miss }}$ resolution, which is equivalent to the role of $p_{\mathrm{T}}^{\text {miss }}$ in the SRs. In order to enhance the purity of the CRs, specific additional selection criteria are applied. For the charged-lepton CRs, at least one of the leptons is required to pass a more strict set of quality criteria and have $p_{\mathrm{T}}>40(20) \mathrm{GeV}$ electrons (muons), while the photon in the photon $\mathrm{CR}$ is required to have $p_{\mathrm{T}}>230 \mathrm{GeV}$ in order to 
ensure high trigger efficiency. Additionally, events in the single-lepton CRs are required to have a transverse mass $m_{\mathrm{T}}=\sqrt{2 p_{\mathrm{T}}^{\text {miss }} p_{\mathrm{T}}^{\ell}\left(1-\cos \left[\Delta \phi\left(\vec{p}_{\mathrm{T}}^{\text {miss }}, \vec{p}_{\mathrm{T}}^{\ell}\right)\right]\right)}<160 \mathrm{GeV}$, and events in the single-electron $\mathrm{CR}$ are required to have $p_{\mathrm{T}}^{\text {miss }}>50 \mathrm{GeV}$ in order to reject contributions from QCD multijet events. Finally, in order to enrich the dilepton CRs with $\mathrm{Z}$ events, the two leptons are required to have opposite signs and to have an invariant mass in the range $60<m_{\ell \ell}<120 \mathrm{GeV}$, consistent with the mass of the $\mathrm{Z}$ boson [71].

The event selection criteria for the signal regions of the different analysis categories, and the topological selection differences between regions in the same category are respectively summarized in tables 1 and 2 in appendix A.1.

\section{Background estimation}

Background estimation and signal extraction are performed simultaneously, using a joint maximum likelihood (ML) fit across all SRs and the corresponding single-lepton, dilepton, and photon CRs. For each analysis category, a likelihood function is constructed to model the expected background contributions in each recoil variable bin of the SR and CRs, as well as the expected signal yield in each bin of the SR. The best fit background model, as well as the best fit signal strength, are obtained by maximizing the joint likelihood function of all categories.

\subsection{Likelihood function}

The likelihood function is defined in the same way as described in ref. [72] and previously used in ref. [22]. Separate approaches are adopted to estimate the dominant ( $Z+$ jets, $\mathrm{W}+$ jets, $\gamma+$ jets) and subdominant ( $\mathrm{t} \overline{\mathrm{t}}$, diboson, and QCD multijet) backgrounds.

The predictions for the dominant backgrounds are based on the yield of $Z \rightarrow v v$ events in each bin of the SR. The per-bin yields for this process are defined as free parameters of the likelihood function. The yields for the $\mathrm{W}+$ jets contribution to the SR, as well as the yields of the $\gamma+$ jets process in the photon $\mathrm{CR}$ and the $\mathrm{Z} \rightarrow \ell \ell$ process in the dilepton CRs, are defined relative to the $\mathrm{Z} \rightarrow \mathrm{v} v$ yields by introducing a set of per-bin transfer factors. The yields of $\mathrm{W} \rightarrow \ell v$ events in the single-lepton CRs are similarly related via transfer factors to the $\mathrm{W} \rightarrow \ell v$ event yields in the SRs. This choice of transfer factors takes into account the correlations between the $\mathrm{V}+$ jets background contributions in all regions. In all cases, the central values of the transfer factors are obtained from the ratios of the simulated recoil spectra of the respective processes in the SRs to those in CRs. For the minor backgrounds, such as t $\bar{t}$ and QCD multijet production, the nominal expected yield per region is obtained directly from simulation (top quark and diboson backgrounds, as well as QCD multijet production in the single-lepton CRs) or by dedicated estimates based on control samples in data (QCD multijet production in the SRs and photon CRs). Contributions from triboson processes are negligible.

Systematic uncertainties are incorporated in the likelihood function as nuisance parameters, as described in more detail below. In the case of the $\mathrm{V}+$ jets processes, the nuisance parameters affect the values of the transfer factors in each recoil variable bin and thus control the ratios of the contributions from different processes, as well as the ratios of the yields 
in the SRs to those in various CRs. For the subdominant background processes, the yields in each bin are directly parameterized in terms of the nuisance parameters. The final free parameter of the likelihood function is the signal strength modifier $\mu$, which - for a given signal hypothesis - controls the signal normalization relative to the theoretical cross section.

The likelihood method relies on the accurate predictions of the ratios between the dominant backgrounds in the SRs and CRs, as well as on the absolute normalization and shape of the recoil distributions for the subdominant backgrounds. To achieve the most accurate possible predictions for these quantities, weights are applied to each simulated event to take into account both experimental and theoretical effects not present in the MC simulated samples. The experimental corrections are related to the trigger efficiencies, identification and reconstruction efficiencies of charged leptons, photons and b-tagged jets, and the pileup distribution in simulation. Theoretical corrections are applied to the $\mathrm{V}+$ jets processes in order to model the effects of NLO terms in the perturbative EW corrections [73]. The corrections are parameterized as functions of the generator-level boson $p_{\mathrm{T}}$ and are evaluated separately for the $\mathrm{W}(\ell v)+$ jets, $\mathrm{Z}(\ell \ell)+$ jets, and $\gamma+$ jets processes. For the diboson processes (WW, WZ, and ZZ), EW and QCD NLO corrections are applied differentially in the boson $p_{\mathrm{T}}$. The EW corrections are obtained from ref. [74], while the QCD corrections are derived from simulated samples generated with MADGRAPH5_aMC@NLO and POWHEG. The EW NLO corrections for the $\mathrm{W} \gamma$ and $\mathrm{Z} \gamma$ processes are similarly obtained from refs. [75, 76].

The validity of the predictions is checked by considering the differential ratio of yields in the CRs. The yield ratio serves as a proxy for the ratios of the different $\mathrm{V}+$ jets processes, which the fit relies on. The yield ratios between the dilepton and single-lepton CRs, and between the dilepton and photon CRs are shown in figures 2 and 3, respectively. Good agreement is observed between prediction and data. In the monojet categories, it is found that the rate of $\mathrm{W} \rightarrow \ell v$ events is initially underpredicted relative to $\mathrm{Z} \rightarrow \ell \ell$ and $\gamma$ events. This underprediction is corrected in the ML fit, mostly via an adjustment of the nuisance parameters related to the experimental efficiencies for leptons and photons, as well as those related to the noncanceling components of the QCD higher-order corrections.

\subsection{Estimation of the QCD multijet background}

The contributions from QCD multijet events in each SR and the corresponding photon $\mathrm{CR}$ are estimated from data. Multijet events do not carry large intrinsic $p_{\mathrm{T}}^{\text {miss }}$, and therefore could only contribute to the SR if one of the hadronic jets in an event is significantly misreconstructed or partially lost, leading to the $\vec{p}_{\mathrm{T}}^{\text {miss }}$ vector and the transverse momentum vector of the jet being aligned. The contribution from such events is estimated from a CR that is enriched in multijet events by inverting the requirement on $\Delta \phi\left(\vec{p}_{\mathrm{T}}^{\text {miss }}, \vec{p}_{\mathrm{T}}^{\mathrm{j}}\right)$ relative to the $\mathrm{SR}$. The recoil spectrum of multijet events in the SR is obtained by multiplying the spectrum in data in this CR by a transfer factor obtained from simulation. The nonmultijet background components, as predicted from simulation, are subtracted from data before applying the transfer factor. The performance of the method is tested by splitting the low $-\Delta \phi\left(\vec{p}_{\mathrm{T}}^{\text {miss }}, \vec{p}_{\mathrm{T}}^{\mathrm{j}}\right)$ CR into parts across different boundaries in $\Delta \phi\left(\vec{p}_{\mathrm{T}}^{\mathrm{miss}}, \vec{p}_{\mathrm{T}}^{\mathrm{j}}\right.$ ) (e.g., for a boundary of 0.25 , the regions would be $\Delta \phi\left(\vec{p}_{\mathrm{T}}^{\mathrm{miss}}, \vec{p}_{\mathrm{T}}^{\mathrm{j}}\right)<0.25$ and $\left.0.25<\Delta \phi\left(\vec{p}_{\mathrm{T}}^{\text {miss }}, \vec{p}_{\mathrm{T}}^{\dot{j}}\right)<0.5\right)$ and verifying that an estimate based 

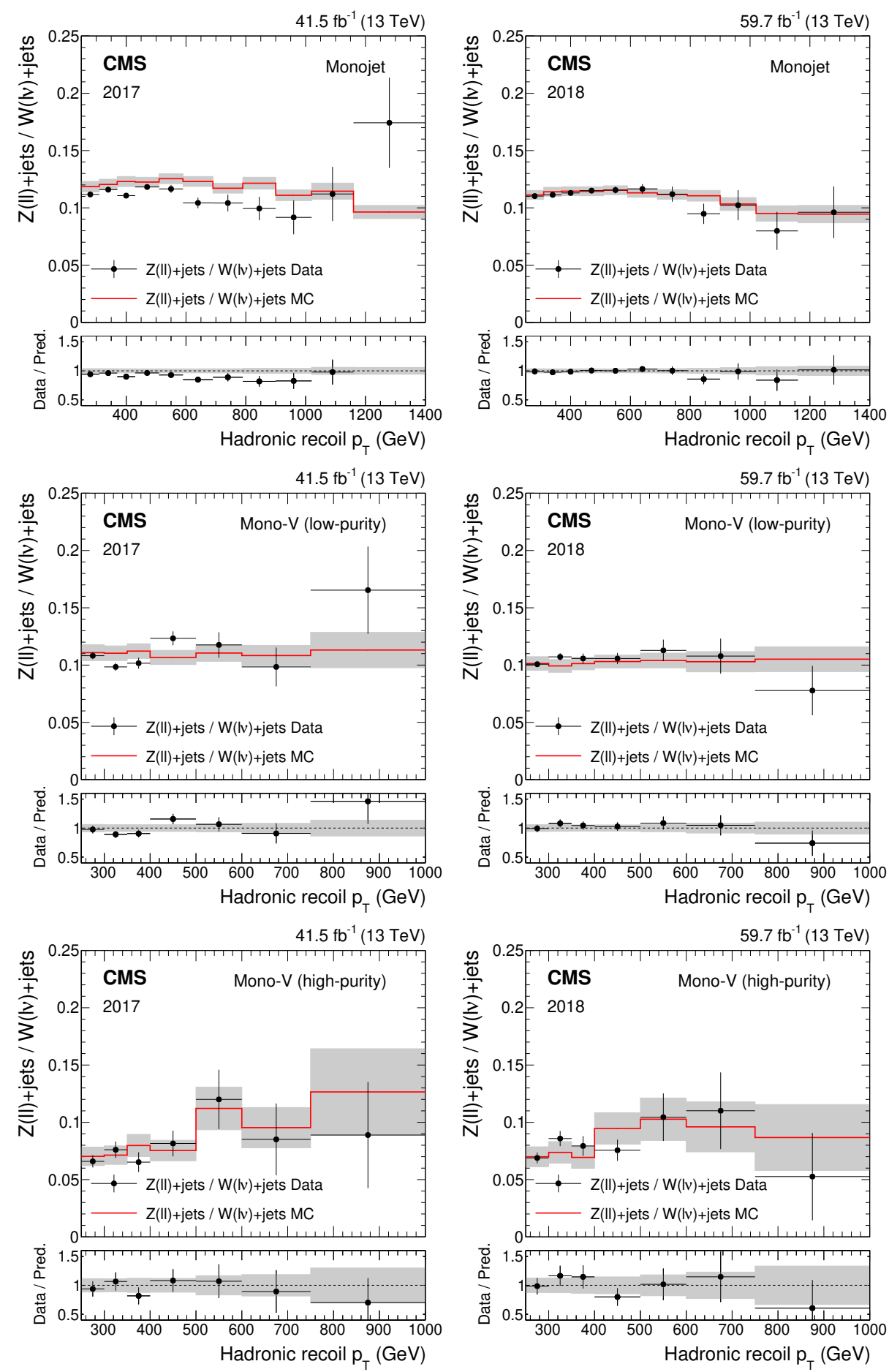

Figure 2. Ratio of the dilepton to single-lepton control region yields predicted using simulation (red solid line), and observed in data (black points). The gray band represents the total uncertainty in the ratio. In the lower panels, the ratio of data over prediction is shown. From upper to lower, the rows show the monojet, low-purity, and high-purity mono- $\mathrm{V}$ categories, while the left (right) column represents the 2017 (2018) data set. 

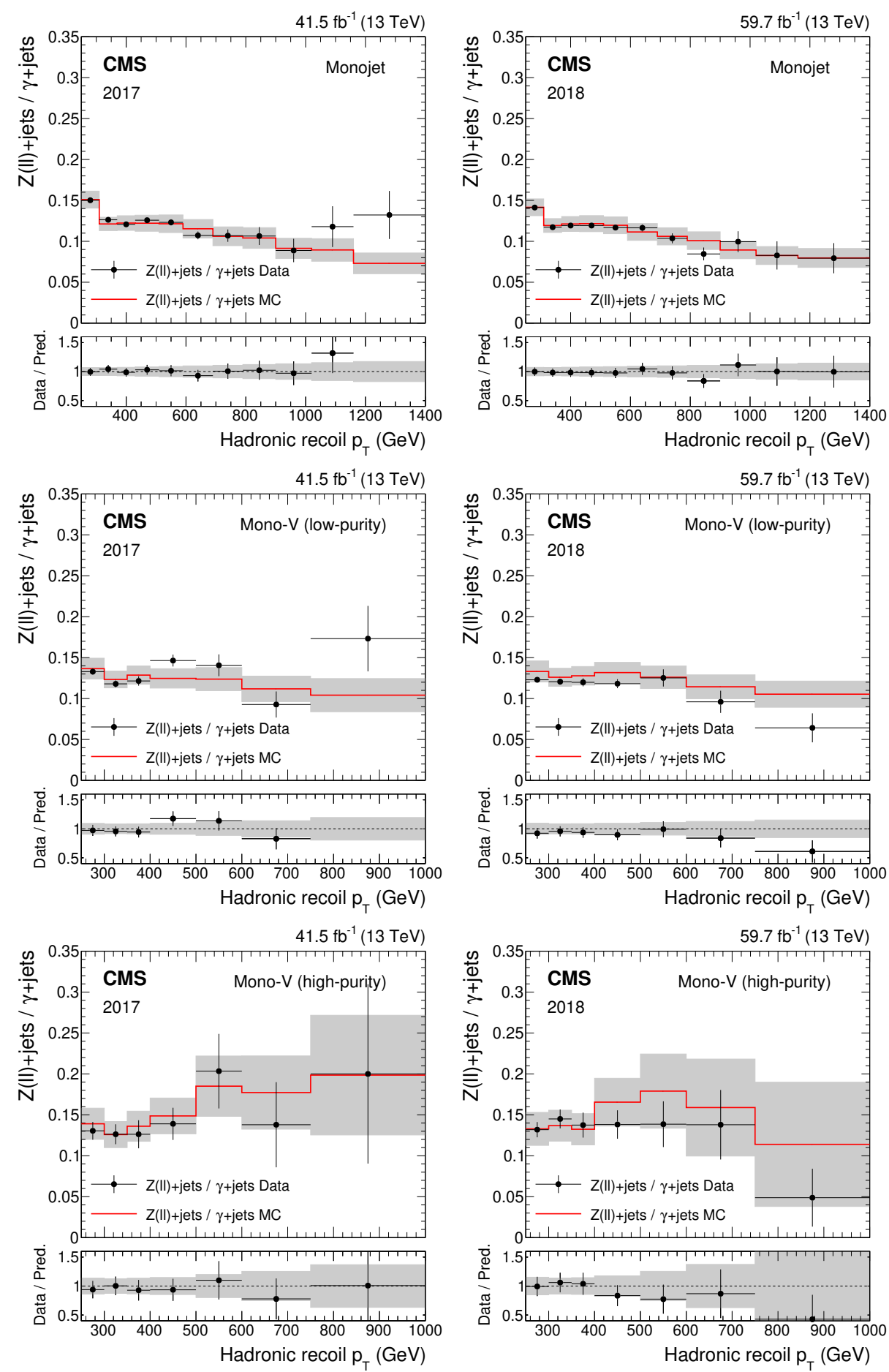

Figure 3. Ratio of the dilepton to photon control region yields predicted using simulation (red solid line), and observed in data (black points). The gray band represents the total uncertainty in the ratio. In the lower panels, the ratio of data over prediction is shown. From upper to lower, the rows show the monojet, low-purity, and high-purity mono- $\mathrm{V}$ categories, while the left (right) column represents the 2017 (2018) data set. 
on the low- $\Delta \phi\left(\vec{p}_{\mathrm{T}}^{\text {miss }}, \vec{p}_{\mathrm{T}}^{\mathrm{j}}\right)$ part of the region $\left(\Delta \phi\left(\vec{p}_{\mathrm{T}}^{\text {miss }}, \vec{p}_{\mathrm{T}}^{\mathrm{j}}\right)<0.25\right.$ in the above example $)$ can correctly predict the QCD multijet background contribution in the high- $\Delta \phi\left(\vec{p}_{\mathrm{T}}^{\mathrm{miss}}, \vec{p}_{\mathrm{T}}^{\mathrm{j}}\right)$ part $\left(0.25<\Delta \phi\left(\vec{p}_{\mathrm{T}}^{\text {miss }}, \vec{p}_{\mathrm{T}}^{\mathrm{j}}\right)<0.5\right)$. The method is found to predict correctly the QCD background contribution to approximately $25 \%$ for various choices of $\Delta \phi\left(\vec{p}_{\mathrm{T}}^{\mathrm{miss}}, \vec{p}_{\mathrm{T}}^{\mathrm{j}}\right)$ boundaries, a value which is assigned as a normalization uncertainty in the QCD multijet background estimate in the SR. Uncertainties related to the finite size of multijet samples, as well as to the choice of the transfer factor binning, are taken into account and may affect the normalization and shape of the background estimate by between 10 and $50 \%$ depending on $p_{\mathrm{T}}^{\text {miss }}$.

In the photon $\mathrm{CR}$, multijet events can contribute if a jet is misreconstructed as an isolated photon. The fraction of photons resulting from jet misreconstruction is estimated from the distribution of the lateral shower width of the photons. The distribution of this variable shows a characteristic peak for genuine photons, while being significantly more flat for the contribution from jets misreconstructed as photons. A template fit is performed to the distribution in data in order to extract the relative contributions of the two components. Templates for genuine photons are obtained from simulation, while templates for misreconstructed jets are taken from a CR in data with an inverted photon isolation requirement that is enriched in QCD multijet events. The fraction of photons originating from jet misreconstruction is found to range between $3.5 \%$ at $p_{\mathrm{T}}=200 \mathrm{GeV}$ and $1 \%$ at $800 \mathrm{GeV}$. A prediction for the recoil distribution in QCD multijet events in the photon CR is obtained by weighting the photon candidate spectrum in data by the misreconstructed jet fraction evaluated at the respective $p_{\mathrm{T}}$ of the photon candidates. A $25 \%$ uncertainty is assigned to the normalization of the QCD multijet background to account for mismodeling of the shower width in simulation. The uncertainty is estimated by repeating the measurement while varying the binning of the shower width distribution used for fitting, which serves to modulate the effect of the mismodeling. The statistical uncertainty in the determination of the differential recoil shape is taken into account and ranges from less than $1 \%$ at low recoil values up to 10 (20)\% at a recoil value of $1.4 \mathrm{TeV}$ in the 2017 (2018) data set.

\subsection{Systematic uncertainties}

The inputs to the ML fit are subject to various experimental and theoretical uncertainties. The overall experimental uncertainty is dominated by the uncertainties in the efficiency of identifying and reconstructing lepton and photon candidates, as well as the uncertainty in the trigger efficiency. The uncertainties in the efficiencies of reconstructing and identifying electron candidates are 1.0 and 2.5\%, respectively. For muons, the corresponding uncertainties are 1\%, with an additional 1\% uncertainty in the efficiency of the isolation criteria. Finally, for photons, the uncertainty in the reconstruction efficiency is negligible, and the uncertainty in the identification efficiency ranges between $4 \%$ at $p_{\mathrm{T}}=200 \mathrm{GeV}$ and $12 \%$ at $1 \mathrm{TeV}$. The uncertainties in the identification efficiency of lepton candidates are further propagated to the estimate of the contribution from background processes in the SRs, where events with identified leptons are rejected. These uncertainties predominantly affect the $\mathrm{W} \rightarrow \ell v$ process, and their magnitude is taken to be $1-2 \%$ of the total $\mathrm{W} \rightarrow \ell v$ yield for the identification of $\tau$ leptons, $1.5 \%$ for electrons, and less than $0.5 \%$ for muons. The uncertainty in the photon energy calibration modeling is $1 \%$ of the photon momentum, 
leading to an effect on the background yield in the photon control region of up to $3 \%$ at low recoil values. The uncertainty in the $\mathrm{b}$ tagging efficiency leads to an uncertainty of $6 \%$ in the normalization of background processes with top quarks, and $2 \%$ in the normalization of the diboson and QCD multijet processes. The uncertainties in the trigger efficiency are $2 \%$ for both the electron or photon triggers, and $1 \%$ per identified muon for the $p_{\mathrm{T}}^{\text {miss }}$ trigger for recoil values of less than $400 \mathrm{GeV}$, and negligible above this threshold. The muon multiplicity dependence of the $p_{\mathrm{T}}^{\text {miss }}$ trigger uncertainty reflects the differences in the reconstruction of muons at the trigger and offline levels, which affect the calculation of the hadronic recoil value. Uncertainties of $75 \%$ are assigned to the normalization of the QCD multijet background contributions in the single-lepton regions, which are estimated from LO simulation. Finally, additional uncertainties of $20 \%$ each are assigned to the rate of the Drell-Yan events entering the single-lepton CRs and of the $\gamma+$ jets events entering the single-electron CRs.

The theoretical uncertainties in the transfer factors related to higher-order effects in the QCD and EW perturbative expansions are calculated according to the prescription given in ref. [73] and implemented, as described in ref. [22]. The uncertainty related to the modeling of PDFs is estimated using the replicas provided in the PDF4LHC15 PDF set [77-80]. Additionally, uncertainties of $10 \%$ each are assigned to the cross sections of the diboson and top quark processes, and a further $10 \%$ normalization uncertainty is assigned to account for the differences in the $p_{\mathrm{T}}$ spectrum of simulated and observed top quark events [81]. For the diboson and $\mathrm{V} \gamma$ processes, additional uncertainties related to unknown mixed QCD-EW NLO corrections are estimated based on the product of the individual EW and QCD correction terms. These uncertainties range between 1 and 10\%, depending on the process and boson $p_{\mathrm{T}}$.

The likelihood functions obtained for the monojet and mono- $\mathrm{V}$ categories, as well as for the two data taking years, are combined in order to maximize the statistical power of the analysis. The results based on the data set analyzed here, which corresponds to an integrated luminosity of $101 \mathrm{fb}^{-1}$, are further combined with the results of an earlier analysis [22] based on a data set collected at the same center-of-mass energy in 2016 and corresponding to an integrated luminosity of $36 \mathrm{fb}^{-1}$. The combination is performed by defining a combined likelihood describing all the analysis regions in all data sets. For this purpose, the effects of all theoretical uncertainties are assumed to be correlated. Most experimental uncertainties are dominated by the inherent precision of auxiliary measurements specific to each data set and are thus assumed to be uncorrelated between different data taking years. The experimental uncertainties related to the JES and JER, as well as those related to the determination of the integrated luminosity are partially correlated between the data taking years, which is taken into account by splitting the total uncertainty into its correlated and uncorrelated components. In order to harmonize the theoretical signal treatment between the data sets, the signal templates from ref. [22] are replaced by the templates derived from simulated samples with generator configurations identical to those used in the analysis of the more recent data sets. Use of the more accurate generator worsens the excluded cross sections based on the 2016 data set alone by up to $13 \%$, depending on the signal hypothesis. The effect is reduced to a few percent level in the fully combined final result. 


\section{Results and interpretation}

The ML fit is performed by combining the analysis categories as well as the 2017 and 2018 data sets. The $p_{\mathrm{T}}^{\text {miss }}$ distributions in the SRs before ("pre-fit") and after ("post-fit") the fit are shown in figure 4 for the monojet category and in figure 5 for the low-purity and high-purity mono- $\mathrm{V}$ categories. In all cases, good agreement is observed between the background-only post-fit result and the data. The corresponding distributions for the CRs are shown in figures $13-18$ in appendix A.2.

In the following, signal strength exclusion limits are presented for different signal hypotheses. Unless explicitly stated, all data sets and categories are included. The exclusion limits are calculated using the asymptotic approximation of the $\mathrm{CL}_{\mathrm{s}}$ method [82-84]. In this method, a signal-plus-background fit is performed for each signal hypothesis in addition to the background-only fit. In the signal fits, the nuisance parameters are profiled, and the resulting best fit nuisance parameters vary for the different signal hypotheses. Consequently, different nonzero best fit values for the signal strength can be obtained for different signals even if the background-only fit succeeds in modeling the data. In the exclusion limits, this feature is represented by differences between the observed and expected limits.

\subsection{Higgs portal interpretation}

The results are interpreted in terms of the exclusion limits at $95 \%$ confidence level (CL) on the branching fraction of an otherwise SM-like Higgs boson to particles without detectable detector interactions (invisible decays). The limits are derived assuming the SM production cross section for the Higgs boson [60]. In the monojet category, values of $\mathcal{B}(\mathrm{H} \rightarrow$ inv.) larger than $59.6 \%$ are excluded $(36.2 \%$ expected). In the combination of the mono- $\mathrm{V}$ categories, branching fractions of more than $37.0 \%$ are excluded (31.0\% expected). Finally, the combination of all categories yields an exclusion limit of $\mathcal{B}(\mathrm{H} \rightarrow$ inv. $)<27.8 \%(25.3 \%$ expected). These limits are summarized in figure 6 . The result from the combination of the mono- $\mathrm{V}$ and monojet channels exhibits a closer agreement between the expected and observed exclusions than either of the two channels individually. This is a result of correlations in the background model between the categories. A year-by-year breakdown of the sensitivity is shown in figure 19 in appendix A.3. Compared to the previous result in the same channel from ref. [22], which is included here, the exclusion limit is improved by a factor of 1.9 (1.6 expected), and represents the most stringent limit from the combined gluon-fusion and V(qq)H channels to date. The current best limit is $19 \%$ from ref. [9], in which multiple analyses based on data sets of up to $36 \mathrm{fb}^{-1}$ are combined, including ref. [22].

\subsection{Interpretation in a DM simplified model with a colorless mediator}

The results are further interpreted in terms of simplified models of DM production. In a model with a spin-1 mediator, exclusion limits are calculated in the two-dimensional parameter space of the DM and mediator particle masses, $m_{\mathrm{DM}}$ and $m_{\text {med }}$. The coupling between the mediator and the SM quarks is set to a constant value of $g_{\mathrm{q}}=0.25$, the mediator-DM coupling is set to $g_{\chi}=1.0$, and vector and axial-vector type couplings are considered in separate interpretations. The resulting exclusion limits at 95\% CL on 

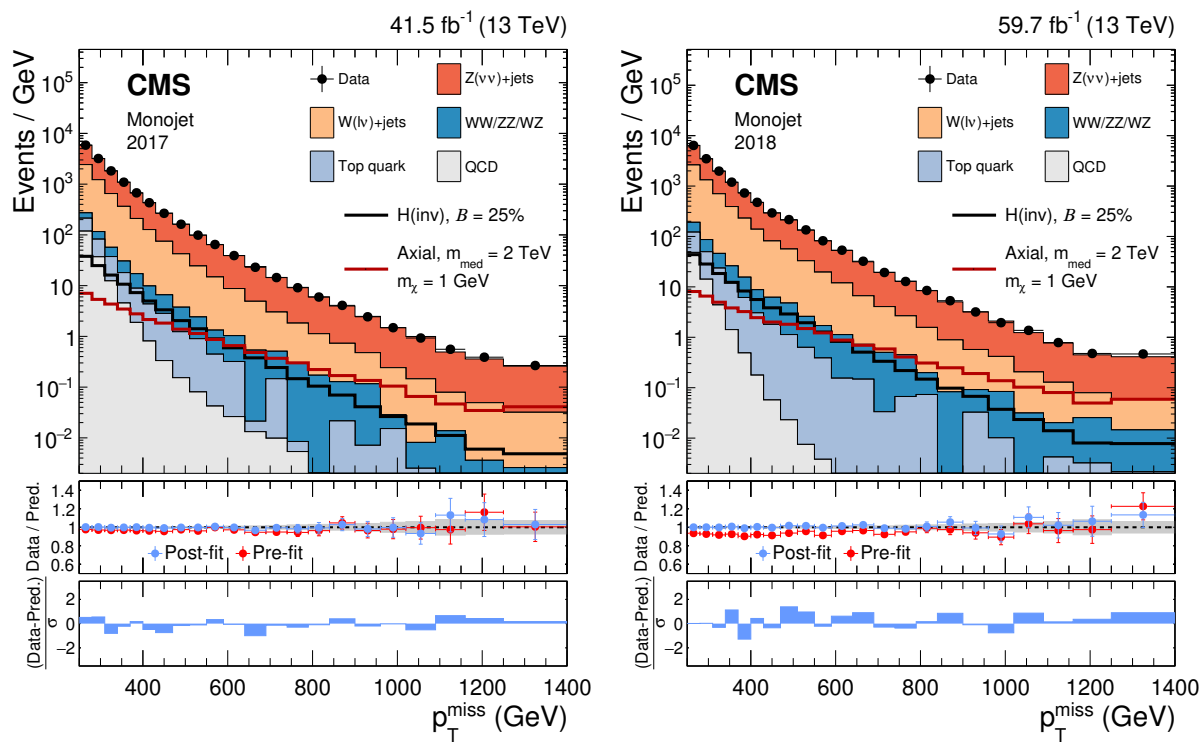

Figure 4. Comparison between data and the background prediction in the monojet signal region before and after the simultaneous fit. The fit includes all control regions and the signal region in all categories and both data taking years, and the background-only fit model is used. The resulting distributions are shown separately for 2017 (left) and 2018 (right). Templates for two signal hypotheses are shown overlaid as black and dark red solid lines. The last bin includes the overflow. In the middle panels, ratios of data to the pre-fit background prediction (red solid points) and post-fit background prediction (blue solid points) are shown. The gray band in the middle panels indicates the post-fit uncertainty after combining all the systematic uncertainties. Finally, the distribution of the pulls, defined as the difference between data and the post-fit background prediction divided by the quadratic sum of the post-fit uncertainty in the prediction and statistical uncertainty in data, is shown in the lower panels.

the signal strength $\mu$ are shown in figure 7 . Values of $m_{\text {med }}$ up to $1.95 \mathrm{TeV}(2.2 \mathrm{TeV}$ expected) are excluded for low $m_{\mathrm{DM}}$ values. The maximum excluded values of $m_{\text {med }}$ decrease with increasing $m_{\mathrm{DM}}$, as the branching fraction of the mediator to DM particle decays diminishes. The dependence of the branching fraction on $m_{\mathrm{DM}}$ is more pronounced in the case of an axial-vector mediator, leading to a reduced maximal exclusion reach in $m_{\mathrm{DM}}$ of $0.7 \mathrm{TeV}$, as opposed to $1 \mathrm{TeV}$ for the vector case. Compared to the results of ref. [22], the combined limits improve the maximal exclusion in terms of the mediator mass by approximately $400 \mathrm{GeV}$, or $20 \%$. In addition to the constraints in the $m_{\mathrm{DM}}-m_{\text {med }}$ plane, we also obtain exclusion limits in the planes of $m_{\text {med }}$ and $g_{\mathrm{q}}$, as well as $m_{\text {med }}$ and $g_{\chi}$, which are shown in figure 8 for the case of axial-vector couplings. The coupling value exclusion is derived analytically from the signal strength exclusion at the default coupling values by rescaling the signal cross section according to the production cross section and decay branching fractions of the mediator, using the formalism of ref. [64]. The DM candidate mass $m_{\mathrm{DM}}$ is fixed to $m_{\text {med }} / 3$. For low mediator masses, values of $g_{\mathrm{q}}\left(g_{\chi}\right)$ as low as 0.018 (0.070) are excluded, providing significant additional insight into the probed parameter space, compared to the mass exclusion for fixed coupling values. Below $m_{\text {med }} \approx 750 \mathrm{GeV}$, 

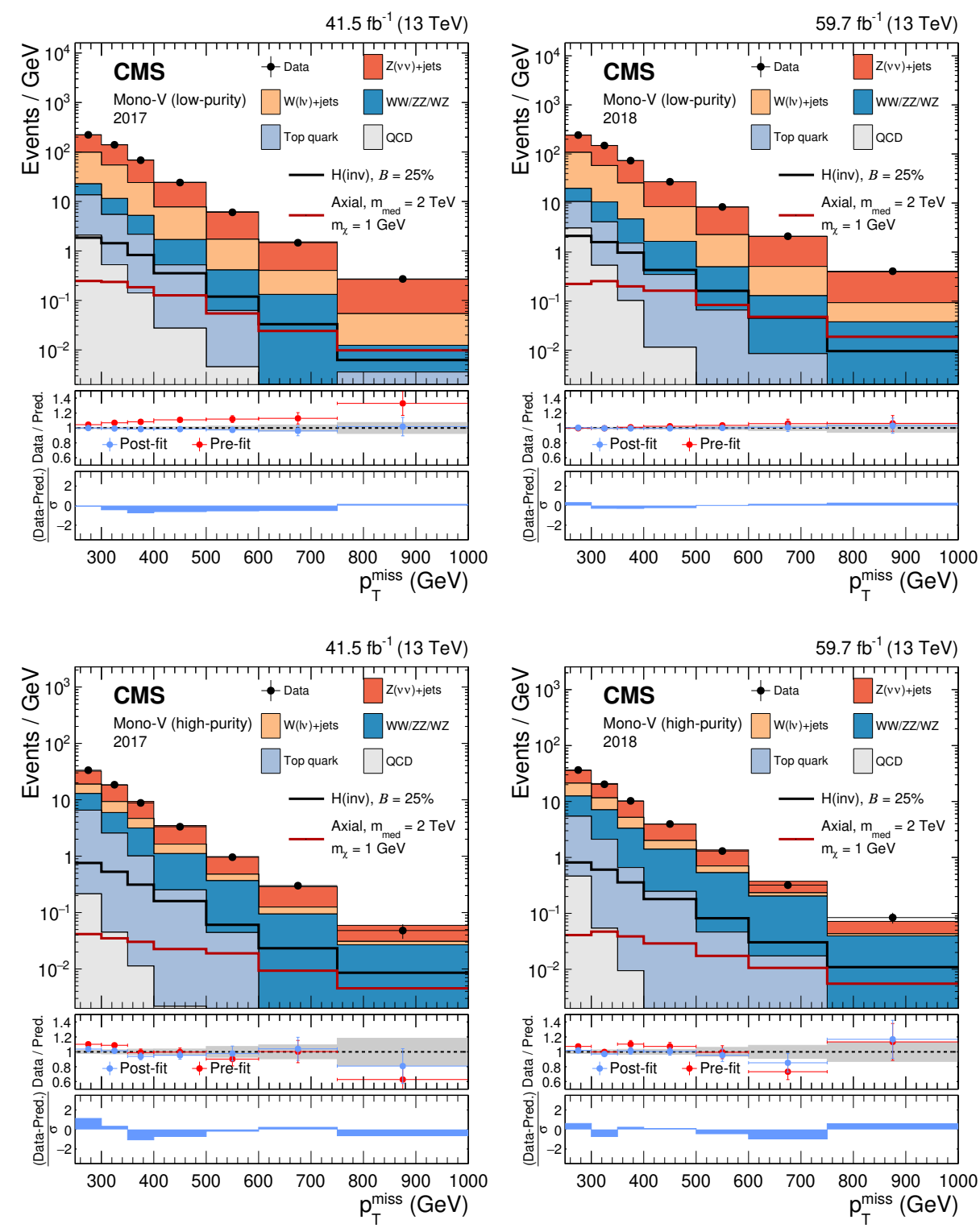

Figure 5. Comparison between data and the background prediction in the mono-V signal regions before and after the simultaneous fit. The fit includes all control regions and the signal region in all categories and both data taking years, and the background-only fit model is used. The resulting distributions are shown separately for 2017 (left column) and 2018 (right column), as well as for the low- and high-purity categories (upper and lower rows, respectively). Templates for two signal hypothesis are shown overlaid as black and dark red solid lines. The last bin includes the overflow. In the middle panels, ratios of data to the pre-fit background prediction (red solid points) and post-fit background prediction (blue solid points) are shown. The gray band in the middle panels indicates the post-fit uncertainty after combining all the systematic uncertainties. Finally, the distribution of the pulls, defined as the difference between data and the post-fit background prediction divided by the quadratic sum of the post-fit uncertainty in the prediction and statistical uncertainty in data, is shown in the lower panels. 


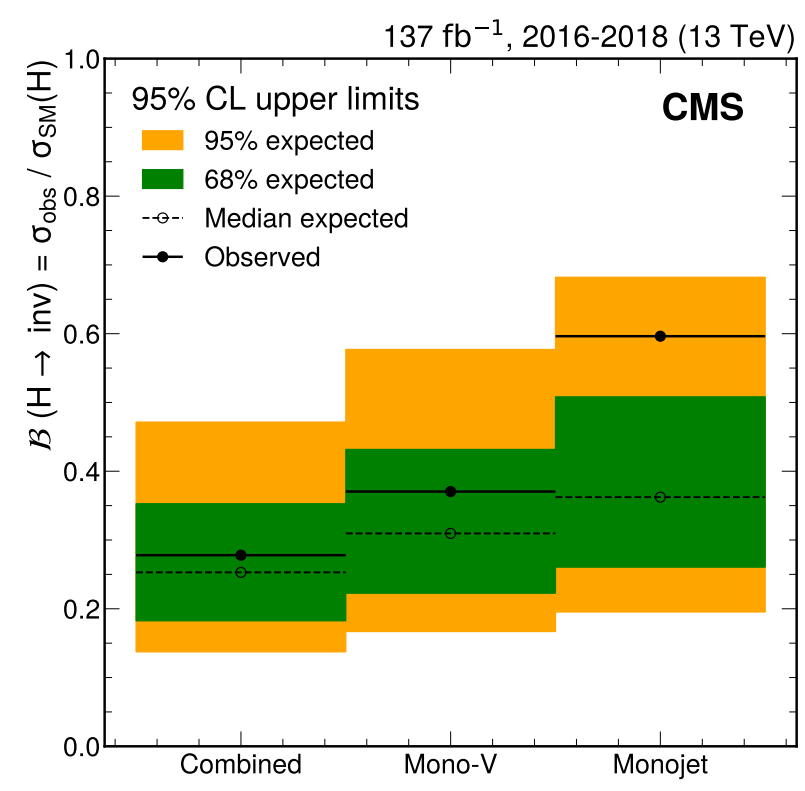

Figure 6. Upper limits at $95 \% \mathrm{CL}$ on the branching fraction $\mathcal{B}$ of the Higgs boson to invisible final states. The results are shown separately for the monojet and mono-V categories, as well as for their combination. The final combined limit is $27.8 \%$ (25.3\% expected).

the constraints on $g_{\mathrm{q}}$ are the strongest to date and exceed the sensitivity of searches for mediators decaying to quarks $[85,86]$. The coupling exclusion result for the vector mediator is similar to the axial-vector case, and is shown in figure 20 in appendix A.4.

The expected upper limits on the signal strength in the case of spin-0 mediators are shown in figure 9. The mediator couplings are assumed to be $g_{\mathrm{q}}=1.0$ and $g_{\chi}=1.0$, and the DM candidate mass is fixed to $1 \mathrm{GeV}$. For scalar mediators, signal strengths larger than 1.2 can be excluded at low mediator mass values of $\approx 50 \mathrm{GeV}$. A pseudoscalar mediator with a mass below $m_{\text {med }}=470 \mathrm{GeV}$ is excluded $(490 \mathrm{GeV}$ expected). In both cases, the signal strength limits show distinctive features around the top quark decay threshold of $m_{\text {med }}=2 m_{\mathrm{t}}$. As the mediator is produced via a top quark loop, the signal cross section is enhanced as the mediator mass approaches the threshold from below. Above the threshold, the decay of the mediator into a pair of top quarks becomes possible, leading to a significant suppression of the branching fraction to DM candidates, and therefore the effective signal cross section. A two-dimensional visualization of the pseudoscalar result in the $m_{\text {med }}-m_{\mathrm{DM}}$ plane is shown in figure 21 in appendix A.5. The constraints on the pseudoscalar model presented here are the most stringent to date. 

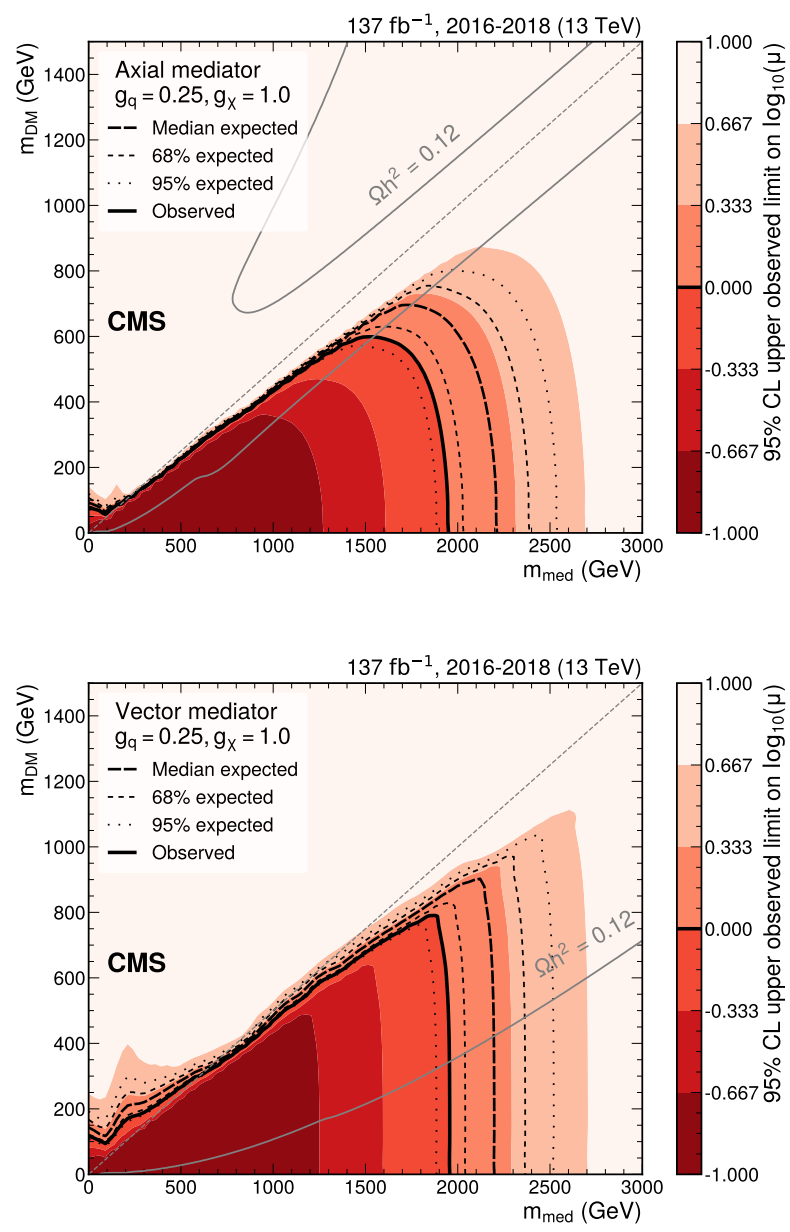

Figure 7. Exclusion limits at $95 \% \mathrm{CL}$ on the signal strength $\mu=\sigma / \sigma_{\text {theo }}$ in the $m_{\text {med }}-m_{\text {DM }}$ plane for coupling values of $g_{\mathrm{q}}=0.25$ and $g_{\chi}=1.0$ for an axial-vector (upper) or vector (lower) mediator. The black solid line indicates the observed exclusion boundary $\mu=1$. The black dashed and dotted lines represent the expected exclusion and the 68 and 95\% CL intervals around the expected boundary, respectively. Parameter combinations with larger values of $\mu$ (indicated by a darker shade in the color scale) are excluded. The observed exclusion reaches up to $m_{\text {med }}=1.95 \mathrm{TeV}(2.2 \mathrm{TeV}$ expected) for low values of $m_{\mathrm{DM}}=1 \mathrm{GeV}$. The gray dashed line indicates the diagonal $m_{\text {med }}=$ $2 m_{\mathrm{DM}}$, above which only off-shell mediator production contributes to the jet $+p_{\mathrm{T}}^{\text {miss }}$ final state. The steep increase of the signal strength limit above the diagonal leads to fluctuations of the exclusion contour, which are due to finite precision in the interpolation method in this region. The gray solid lines represent parameter combinations for which the simplified model reproduces the observed DM relic density in the universe under the assumption of a thermal freeze-out mechanism $[64,87]$. 

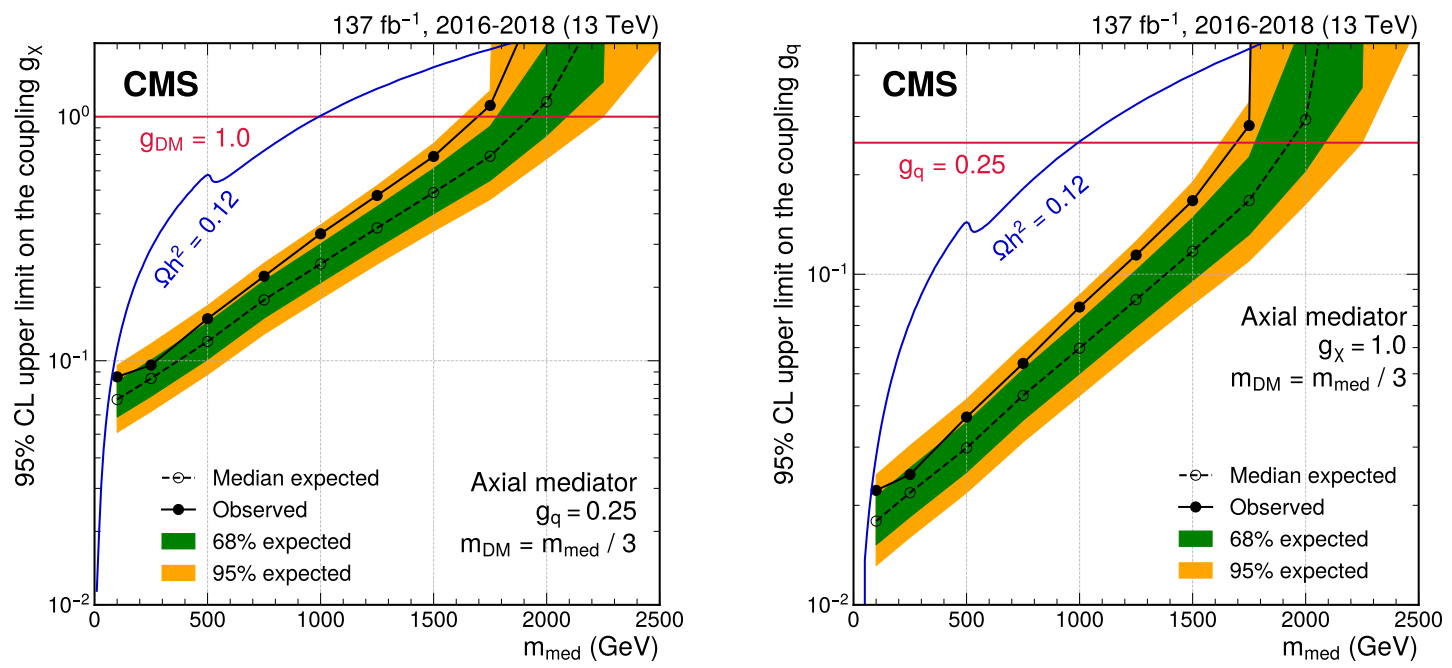

Figure 8. Exclusion limits at 95\% CL on the couplings $g_{\chi}$ (left) and $g_{\mathrm{q}}$ (right) for an axial-vector mediator. In each panel, the result is shown as a function of the mediator mass $m_{\text {med }}$, with the mass of the DM candidate fixed to $m_{\mathrm{DM}}=m_{\text {med }} / 3$. In either case, only one coupling is varied, while the other coupling is fixed at its default value $\left(g_{\mathrm{q}}=0.25\right.$ or $\left.g_{\chi}=1.0\right)$. The blue solid line indicates the parameter combinations for which the simplified model reproduces the observed DM relic density. Around $m_{\mathrm{DM}} \approx m_{\mathrm{top}}$, corresponding to $m_{\text {med }} \approx 500 \mathrm{GeV}, \mathrm{DM}$ annihilation into top quarks becomes possible, leading to a shift in the relic density. The corresponding results for a vector mediator are shown in figure 20 in appendix A.4.
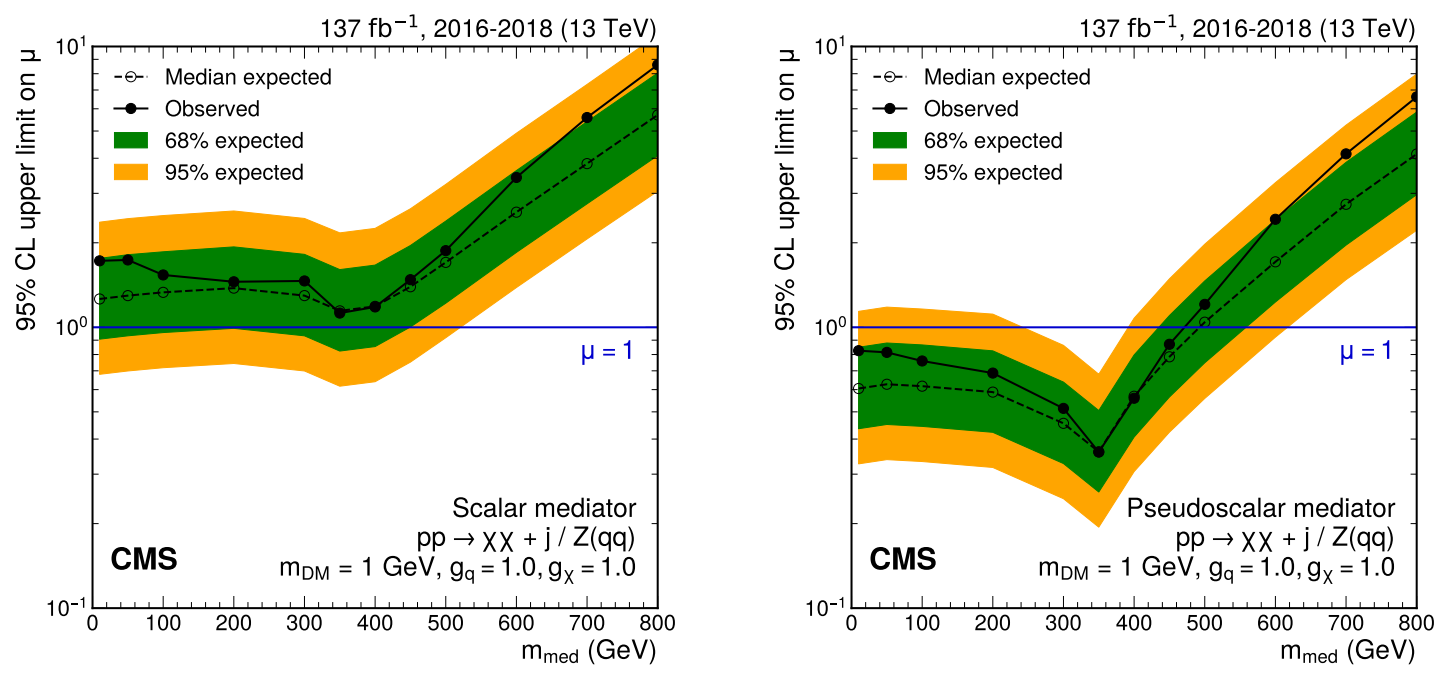

Figure 9. Upper limits at 95\% CL on the signal strength $\mu=\sigma / \sigma_{\text {theo }}$ as a function of $m_{\text {med }}$ for scenarios with scalar (left) and pseudoscalar (right) mediators and coupling values of $g_{\mathrm{q}}=1.0, g_{\chi}=1.0$, for a constant value of $m_{\mathrm{DM}}=1 \mathrm{GeV}$. The blue solid line indicates the exclusion boundary $\mu=1$. In the case of a pseudoscalar mediator, $m_{\text {med }}$ values up to $470 \mathrm{GeV}$ are excluded ( $490 \mathrm{GeV}$ expected). 


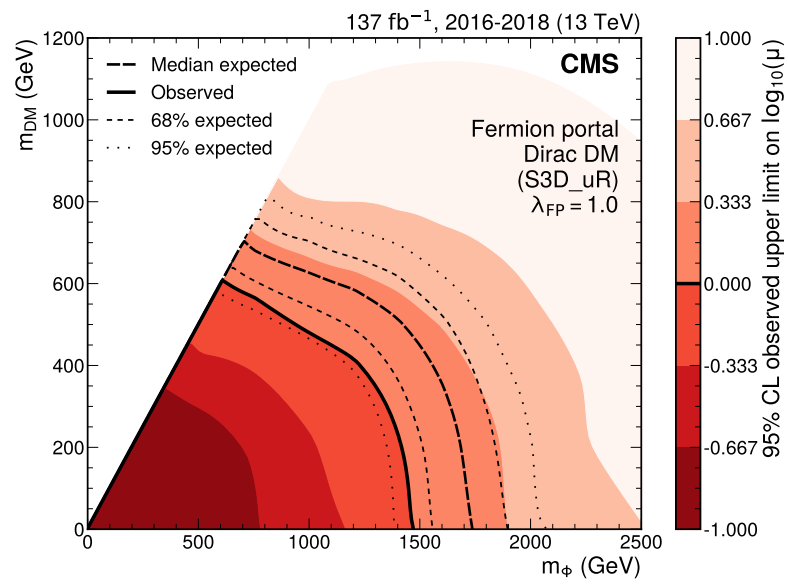

Figure 10. Exclusion limits at 95\% CL in the plane of the mediator mass $m_{\Phi}$ and the DM candidate mass $m_{\mathrm{DM}}$ in the fermion portal model. The black solid line indicates the observed exclusion boundary. The black dashed and dotted lines represent the expected exclusion and the 68 and $95 \%$ CL intervals around the expected boundary, respectively.

\subsection{Fermion portal interpretation}

For the fermion portal model, the results of the analysis are shown in figure 10 in the plane of the mediator mass $m_{\Phi}$ and the DM candidate mass $m_{\mathrm{DM}}$. The coupling between the mediator, DM candidate and the right-handed up quark is set to a constant value of $\lambda_{\mathrm{FP}}=1$. At low $m_{\mathrm{DM}}$ values, mediator masses of up to $1.5 \mathrm{TeV}$ are excluded $(1.7 \mathrm{TeV}$ expected), which are the most stringent constraints on this model to date.

\subsection{The ADD interpretation}

In the ADD scenario, lower limits on the fundamental Planck scale $M_{\mathrm{D}}$ for the number of extra dimensions $d$ ranging from 2 to 7 are shown in figure 11. For the lowest number of extra dimensions considered here, $d=2, M_{\mathrm{D}}$ values of up to $10.7 \mathrm{TeV}$ are excluded (12.2 TeV expected). As the number of extra dimensions increases, the probed $M_{\mathrm{D}}$ value is reduced to $5.2 \mathrm{TeV}$ for $d=7$ (5.6 TeV expected). Compared to the result of ref. [22], these limits represent an improvement of approximately $8 \%$ for low values of $d$ (20\% expected). At larger values, the relative gain in $M_{\mathrm{D}}$ sensitivity is smaller, as a result of the dependence of the signal cross section on $M_{\mathrm{D}}$, which becomes steeper as $d$ increases. The results are also shown in table 3 in appendix A.6.

\subsection{Leptoquark interpretation}

Finally, upper limits are placed on the production cross section of LQs coupled to up quarks and neutrinos with a coupling value $\lambda_{\mathrm{LQ}}$. The branching fraction for the decay of the LQ into an up quark and an electron neutrino is assumed to be $100 \%$ (also referred to as to $\beta=0$ in the literature). The limits are shown in figure 12. Generally, both single and pair LQ production contribute to the signal, with the coupling $\lambda_{\mathrm{LQ}}$ mainly influencing the single production rate. The pair production dominates at lower LQ masses of $m_{\mathrm{LQ}}<1 \mathrm{TeV}$, a region which has already been excluded by previous searches [88]. In the 


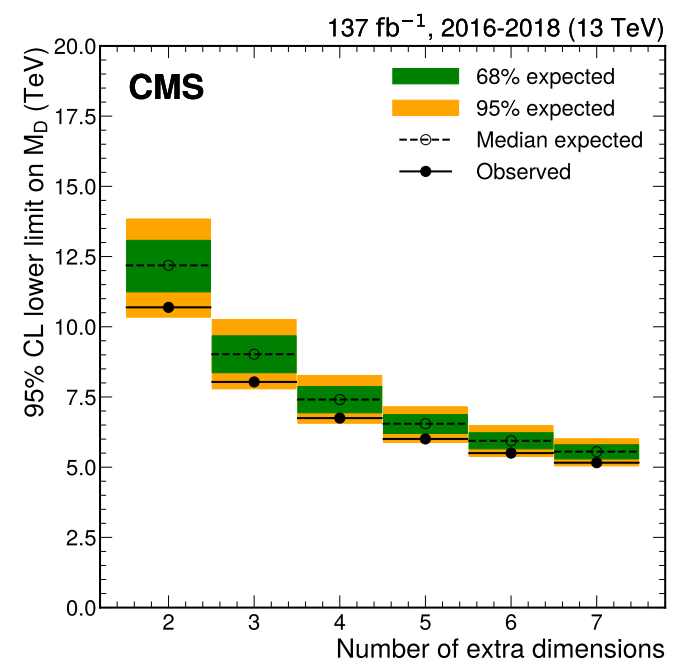

Figure 11. Exclusion limits at $95 \% \mathrm{CL}$ on $M_{\mathrm{D}}$ in the ADD scenario for different values of the number of extra dimensions $d$.

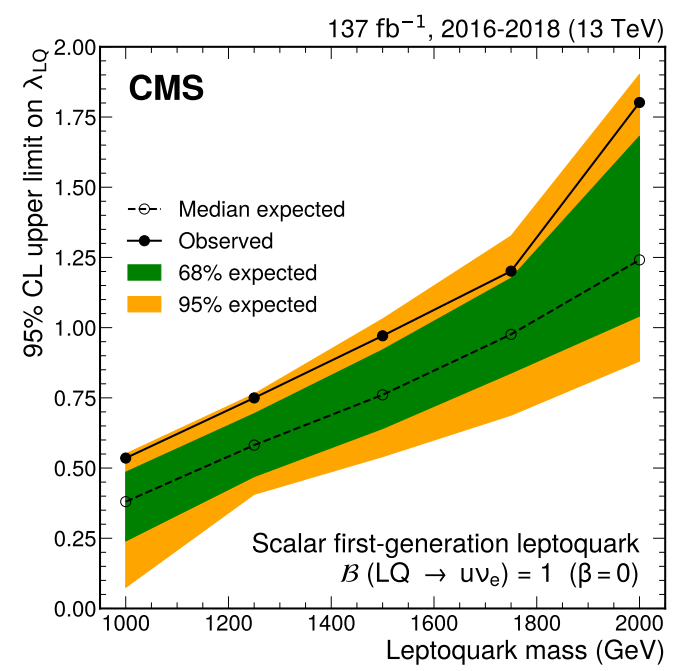

Figure 12. Upper limits at 95\% CL on the leptoquark coupling $\lambda_{\mathrm{LQ}}$ as a function of the leptoquark mass. The branching fraction for the decay of the leptoquark into an electron neutrino and up quark is assumed to be $100 \%(\beta=0)$. The dashed line indicates the median expected exclusion contour.

higher-mass regime, $m_{\mathrm{LQ}}>1 \mathrm{TeV}$, the contribution from single production is increased, providing additional sensitivity to the value of $\lambda_{\mathrm{LQ}}$. The minimum value of the coupling $\lambda_{\mathrm{LQ}}$ excluded ranges from about 0.5 at $m_{\mathrm{LQ}}=1 \mathrm{TeV}(0.4$ expected $)$ to $\lambda_{\mathrm{LQ}}=1.0$ at $m_{\mathrm{LQ}}=1.5 \mathrm{TeV}$ (0.75 expected) and $\lambda_{\mathrm{LQ}}=1.8$ at $2 \mathrm{TeV}$ (1.25 expected), which are the most stringent constraints from a direct search to date. 


\section{Summary}

A search for physics beyond the standard model in events with energetic jets and large missing transverse momentum has been presented. A data set of proton-proton collisions at a center-of-mass energy of $13 \mathrm{TeV}$, corresponding to an integrated luminosity of $101 \mathrm{fb}^{-1}$ is analyzed, and the analysis results are combined with those of an earlier search using an independent data set collected at the same center-of-mass energy, corresponding to an integrated luminosity of $36 \mathrm{fb}^{-1}$ [22]. Separate analysis categories are defined for events with a large-radius jet consistent with a hadronic decay of a $\mathrm{W}$ or a $\mathrm{Z}$ boson, and for events without such a jet. A joint maximum likelihood fit over a combination of signal and control regions is used to constrain standard model (SM) background processes and to extract a possible signal. The data are found to be in good agreement with the fit results, with no evidence for a significant signal contribution. The result is interpreted in terms of exclusion limits at $95 \%$ confidence level on the parameters of a number of models of beyond-the-SM physics. We constrain the branching fraction of the Higgs boson decay to invisible particles to be below $27.8 \%$. In simplified models of the production of dark matter (DM) candidates via a spin-1 s-channel mediator (vector or axial-vector), values of the mediator mass of up to $1.95 \mathrm{TeV}$ are excluded, assuming the couplings of $g_{\mathrm{q}}=0.25$ between the mediator and quarks, and $g_{\chi}=1.0$ between the mediator and the Dirac fermion DM particles. Assuming a fixed ratio $m_{\mathrm{DM}}=m_{\text {med }} / 3$, coupling values as low as $g_{\mathrm{q}}=0.018$ and $g_{\chi}=0.070$ can be excluded for $m_{\text {med }}=100 \mathrm{GeV}$. In a similar model with a pseudoscalar spin-0 mediator, $m_{\text {med }}$ values less than $470 \mathrm{GeV}$ are excluded. The fermion portal model, in which a colored scalar mediator couples to a DM candidate and a right-handed up quark, is excluded for mediator mass values up to $1.5 \mathrm{TeV}$ at low values of the $\mathrm{DM}$ candidate mass $m_{\mathrm{DM}}$, assuming $\lambda_{\mathrm{FP}}=1$. In a model of large extra dimensions, values of the fundamental Planck scale below from 10.7 to $5.2 \mathrm{TeV}$ can be excluded, depending on the number of extra dimensions between 2 and 7. Finally, the production of leptoquarks decaying into the up quark and the electron neutrino is excluded for coupling values between the leptoquarks and the SM fermions larger than 0.5 to 1.8 , for leptoquark masses between 1.0 and $2.0 \mathrm{TeV}$. The constraints on $g_{\mathrm{q}}$ in the spin-1 models, on the mediator and dark matter masses in the pseudoscalar and fermion portal models, and on the leptoquark coupling represent the most stringent bounds to date.

\section{Acknowledgments}

We congratulate our colleagues in the CERN accelerator departments for the excellent performance of the LHC and thank the technical and administrative staffs at CERN and at other CMS institutes for their contributions to the success of the CMS effort. In addition, we gratefully acknowledge the computing centers and personnel of the Worldwide LHC Computing Grid and other centers for delivering so effectively the computing infrastructure essential to our analyses. Finally, we acknowledge the enduring support for the construction and operation of the LHC, the CMS detector, and the supporting computing infrastructure provided by the following funding agencies: BMBWF and FWF (Austria); FNRS and FWO (Belgium); CNPq, CAPES, FAPERJ, FAPERGS, and FAPESP (Brazil); 
MES (Bulgaria); CERN; CAS, MoST, and NSFC (China); MINCIENCIAS (Colombia); MSES and CSF (Croatia); RIF (Cyprus); SENESCYT (Ecuador); MoER, ERC PUT and ERDF (Estonia); Academy of Finland, MEC, and HIP (Finland); CEA and CNRS/IN2P3 (France); BMBF, DFG, and HGF (Germany); GSRT (Greece); NKFIA (Hungary); DAE and DST (India); IPM (Iran); SFI (Ireland); INFN (Italy); MSIP and NRF (Republic of Korea); MES (Latvia); LAS (Lithuania); MOE and UM (Malaysia); BUAP, CINVESTAV, CONACYT, LNS, SEP, and UASLP-FAI (Mexico); MOS (Montenegro); MBIE (New Zealand); PAEC (Pakistan); MSHE and NSC (Poland); FCT (Portugal); JINR (Dubna); MON, RosAtom, RAS, RFBR, and NRC KI (Russia); MESTD (Serbia); SEIDI, CPAN, PCTI, and FEDER (Spain); MOSTR (Sri Lanka); Swiss Funding Agencies (Switzerland); MST (Taipei); ThEPCenter, IPST, STAR, and NSTDA (Thailand); TUBITAK and TAEK (Turkey); NASU (Ukraine); STFC (United Kingdom); DOE and NSF (U.S.A.).

Individuals have received support from the Marie-Curie program and the European Research Council and Horizon 2020 Grant, contract Nos. 675440, 724704, 752730, 758316, 765710, 824093, and COST Action CA16108 (European Union); the Leventis Foundation; the Alfred P. Sloan Foundation; the Alexander von Humboldt Foundation; the Belgian Federal Science Policy Office; the Fonds pour la Formation à la Recherche dans l'Industrie et dans l'Agriculture (FRIA-Belgium); the Agentschap voor Innovatie door Wetenschap en Technologie (IWT-Belgium); the F.R.S.-FNRS and FWO (Belgium) under the "Excellence of Science - EOS" - be.h project n. 30820817; the Beijing Municipal Science \& Technology Commission, No. Z191100007219010; the Ministry of Education, Youth and Sports (MEYS) of the Czech Republic; the Deutsche Forschungsgemeinschaft (DFG), under Germany's Excellence Strategy - EXC 2121 "Quantum Universe" - 390833306, and under project number 400140256 - GRK2497; the Lendület ("Momentum") Program and the János Bolyai Research Scholarship of the Hungarian Academy of Sciences, the New National Excellence Program ÚNKP, the NKFIA research grants 123842, 123959, 124845, 124850, 125105, 128713, 128786, and 129058 (Hungary); the Council of Science and Industrial Research, India; the Latvian Council of Science; the Ministry of Science and Higher Education and the National Science Center, contracts Opus 2014/15/B/ST2/03998 and 2015/19/B/ST2/02861 (Poland); the National Priorities Research Program by Qatar National Research Fund; the Ministry of Science and Higher Education, project no. 07232020-0041 (Russia); the Programa Estatal de Fomento de la Investigación Científica y Técnica de Excelencia María de Maeztu, grant MDM-2015-0509 and the Programa Severo Ochoa del Principado de Asturias; the Stavros Niarchos Foundation (Greece); the Rachadapisek Sompot Fund for Postdoctoral Fellowship, Chulalongkorn University and the Chulalongkorn Academic into Its 2nd Century Project Advancement Project (Thailand); the Kavli Foundation; the Nvidia Corporation; the SuperMicro Corporation; the Welch Foundation, contract C-1845; and the Weston Havens Foundation (U.S.A.). 


\begin{tabular}{|c|c|c|}
\hline Category & Variable / Description & Selection \\
\hline \multirow{9}{*}{ All } & Electron veto & $p_{\mathrm{T}}>10 \mathrm{GeV}$ and $|\eta|<2.5$ \\
\hline & Muon veto & $p_{\mathrm{T}}>10 \mathrm{GeV}$ and $|\eta|<2.4$ \\
\hline & $\tau$ lepton veto & $p_{\mathrm{T}}>18 \mathrm{GeV}$ and $|\eta|<2.3$ \\
\hline & Photon veto & $p_{\mathrm{T}}>15 \mathrm{GeV}$ and $|\eta|<2.5$ \\
\hline & $\mathrm{b}$ jet veto & DeepCSV "medium", $p_{\mathrm{T}}>20 \mathrm{GeV},|\eta|<2.4$ \\
\hline & $p_{\mathrm{T}}^{\mathrm{miss}}$ & $>250 \mathrm{GeV}$ \\
\hline & $\Delta p_{\mathrm{T}}^{\text {miss }}(\mathrm{PF}$-calorimeter $)$ & $<0.5$ \\
\hline & $\Delta \phi(\mathrm{PF}$, charged $)$ & $<2$ \\
\hline & $\Delta \phi\left(\vec{p}_{\mathrm{T}}^{\mathrm{miss}}, \vec{p}_{\mathrm{T}}^{\mathrm{j}}\right)$ & $>0.5$ \\
\hline \multirow[t]{2}{*}{ All (2018) } & Calorimeter failure mitigation (I) & $\begin{array}{r}\text { no AK4 jet with } p_{\mathrm{T}}>30 \mathrm{GeV}, \\
-1.57<\phi<-0.87,-3.0<\eta<-1.3\end{array}$ \\
\hline & Calorimeter failure mitigation (II) & $\phi\left(\vec{p}_{\mathrm{T}}^{\mathrm{miss}}\right) \notin[-1.62,-0.62]$ if $p_{\mathrm{T}}^{\text {miss }}<470 \mathrm{GeV}$ \\
\hline Monojet & Leading AK4 jet & $p_{\mathrm{T}}>100 \mathrm{GeV}$ and $|\eta|<2.4$ \\
\hline Mono-V & Leading AK8 jet & $\begin{array}{r}p_{\mathrm{T}}>250 \mathrm{GeV},|\eta|<2.4,65<m_{\mathrm{SD}}<120 \mathrm{GeV} \\
\text { Subcategorization based on DEEPAK8 score }\end{array}$ \\
\hline
\end{tabular}

Table 1. Summary of the common selection requirements for mono-V and monojet categories. For the control region selections, the requirements on $p_{\mathrm{T}}^{\text {miss }}$ and $\Delta \phi\left(\vec{p}_{\mathrm{T}}^{\text {miss }}, \vec{p}_{\mathrm{T}}^{\mathrm{j}}\right)$ are replaced by the equivalent selections based on the hadronic recoil, which is calculated as the vectorial sum of the $\vec{p}_{\mathrm{T}}^{\text {miss }}$ and the respective lepton or photon transverse momenta used to define the control region selection. The $\Delta p_{\mathrm{T}}^{\text {miss }}(\mathrm{PF}$-calorimeter $)$ and $\Delta \phi(\mathrm{PF}$, charged $)$ requirements are always evaluated based on $p_{\mathrm{T}}^{\mathrm{miss}}$, and not the hadronic recoil.

\section{A Additional figures and tables}

\section{A.1 Event selection summary tables}

The event selection criteria for the signal regions of the different analysis categories are summarized in table 1. The topological selection differences between regions in the same category are shown in table 2. 


\begin{tabular}{lccccr}
\hline Region type & \multicolumn{2}{c}{ Multiplicities } & Trigger & $\begin{array}{r}\text { Special selection } \\
\text { (relative to signal) }\end{array}$ \\
& e & $\mu$ & $\gamma$ & & - \\
Signal & 0 & 0 & 0 & $p_{\mathrm{T}}^{\text {miss }}$ & \\
Single electron & 1 & 0 & 0 & Electron $/$ photon & $m_{\mathrm{T}}<160 \mathrm{GeV}, p_{\mathrm{T}}^{\mathrm{miss}}>60 \mathrm{GeV}$ \\
Single muon & 0 & 1 & 0 & $p_{\mathrm{T}}^{\text {miss }}$ & $m_{\mathrm{T}}<160 \mathrm{GeV}$ \\
Dielectron & 2 & 0 & 0 & Electron $/$ photon & $60<m(\ell \ell)<120 \mathrm{GeV}$ \\
Dimuon & 0 & 2 & 0 & $p_{\mathrm{T}}^{\text {miss }}$ & $60<m(\ell \ell)<120 \mathrm{GeV}$ \\
Photon & 0 & 0 & 1 & Photon & \\
\hline
\end{tabular}

Table 2. Summary of the topological selections used for different regions in the same category. Note that the trigger-level $p_{\mathrm{T}}^{\text {miss }}$ calculation does not take into account muons, which makes the $p_{\mathrm{T}}^{\text {miss }}$ based trigger equally suitable for the signal region and muon-based control regions.

\section{A.2 Hadronic recoil distributions in the control regions}

The maximum likelihood fit used to determine signal and background contributions is performed including control regions in data. In each of the control regions, the hadronic recoil, defined as the vectorial sum of $\vec{p}_{\mathrm{T}}^{\text {miss }}$ and the transverse components of the selected lepton or photon momenta, is used as a proxy for $p_{\mathrm{T}}^{\mathrm{miss}}$ in the signal region. The recoil distributions for all control regions in all categories are shown in figures $13-18$. 

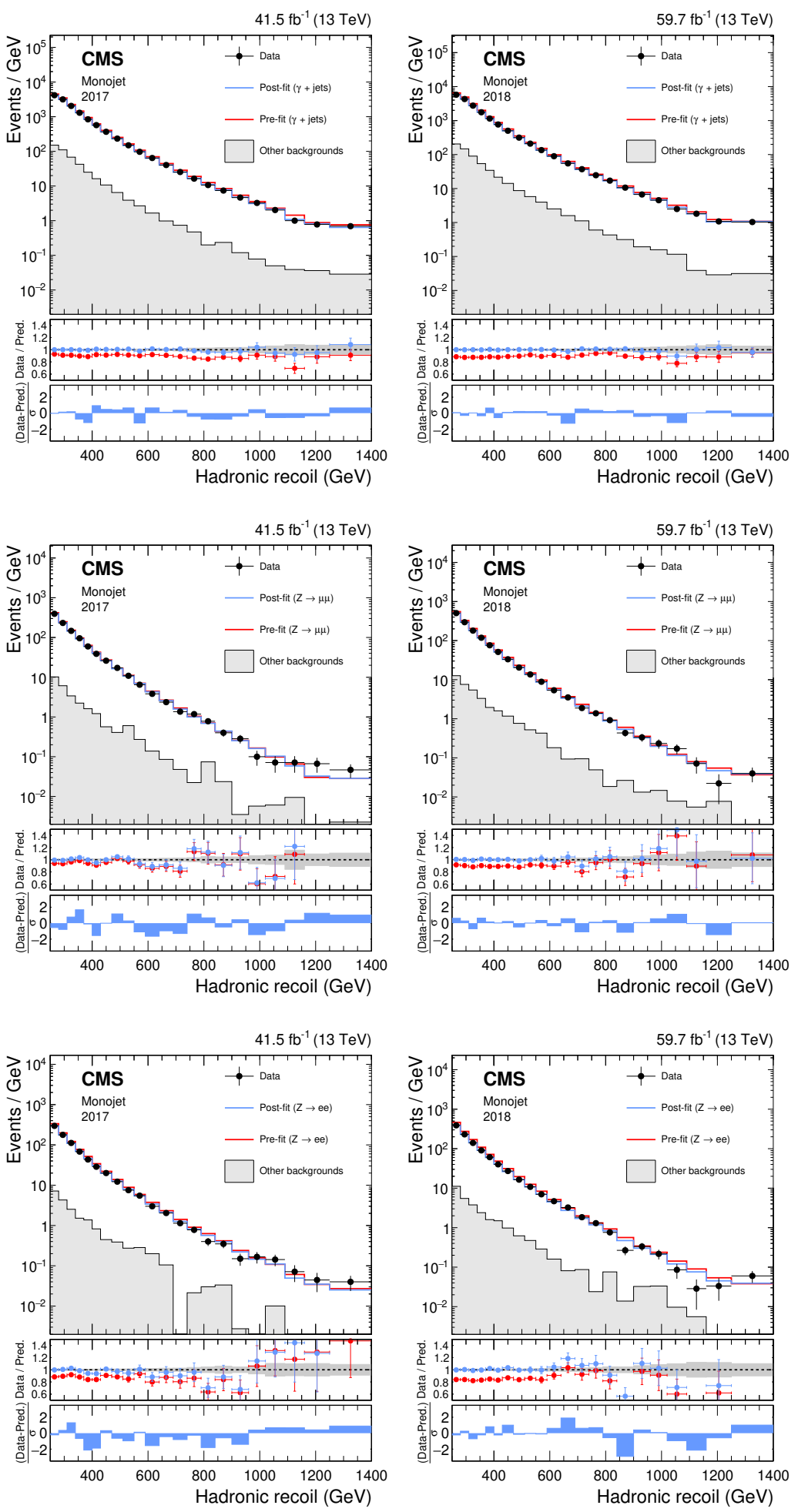

Figure 13. Hadronic recoil distributions in the photon (upper), dimuon (middle) and dielectron control regions (lower) in the monojet category. The "Other backgrounds" include QCD multijet production (photon control region), and top quark, diboson, and $\mathrm{W}+$ jets processes (dimuon and dielectron control regions). 

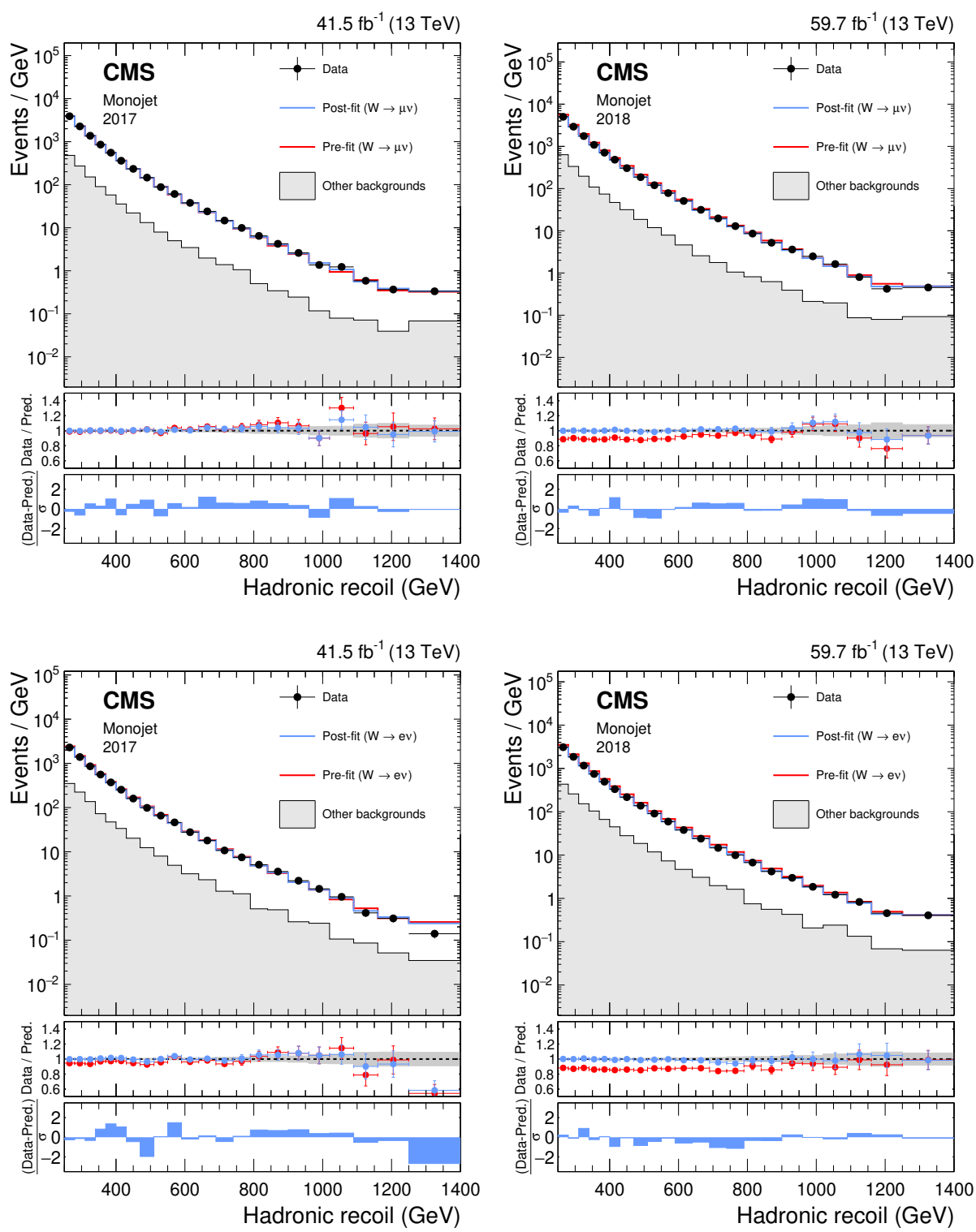

Figure 14. Hadronic recoil distributions in the single muon (upper), and single electron regions (lower) in the monojet category. The "Other backgrounds" include top quark, diboson, and QCD multijet processes. 

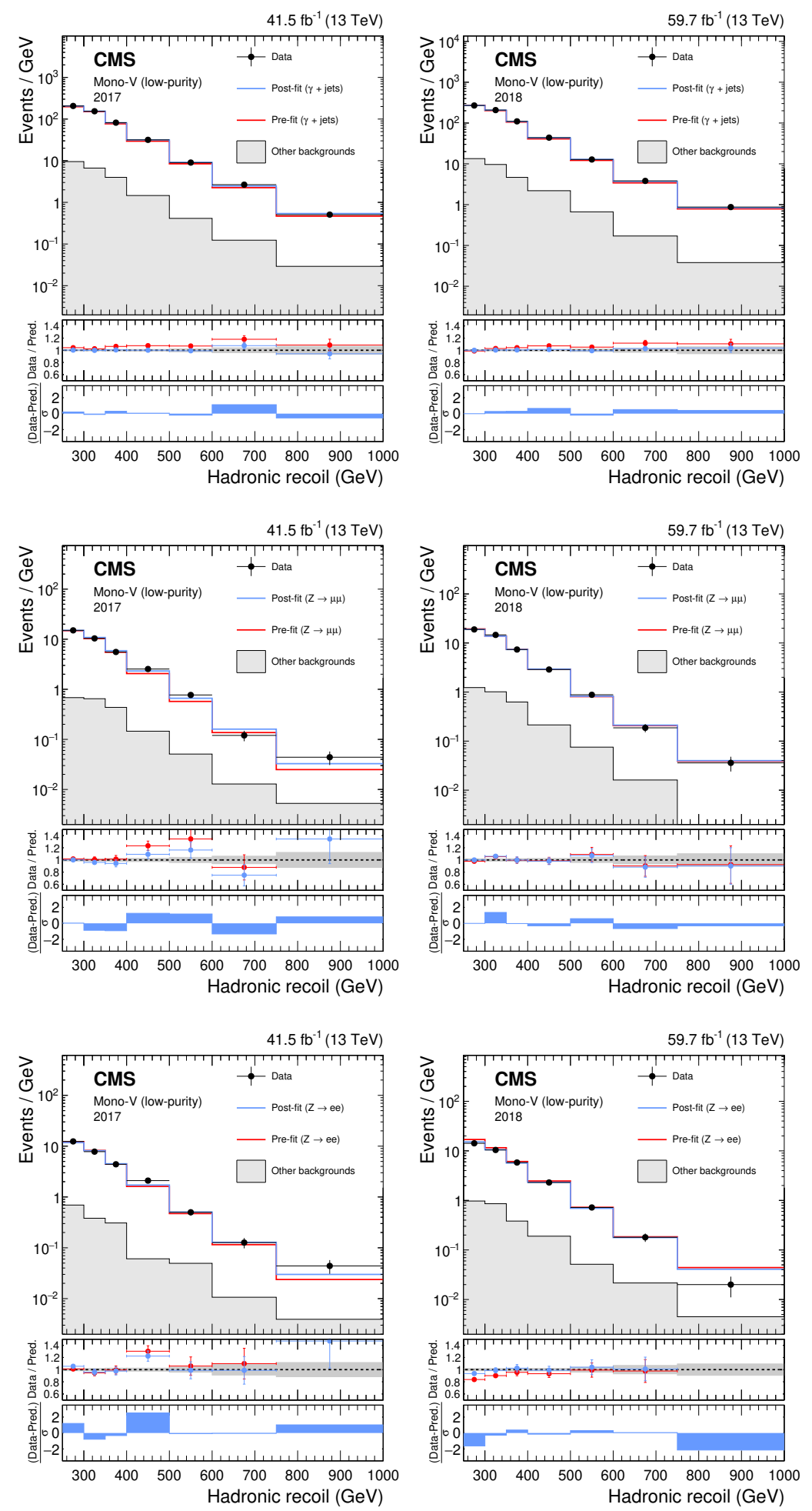

Figure 15. Hadronic recoil distributions in the photon (upper), dimuon (middle) and dielectron control regions (lower) in the low-purity mono-V category. The "Other backgrounds" include QCD multijet production (photon control region), and top quark, diboson, and $\mathrm{W}+$ jets processes (dimuon and dielectron control regions). 

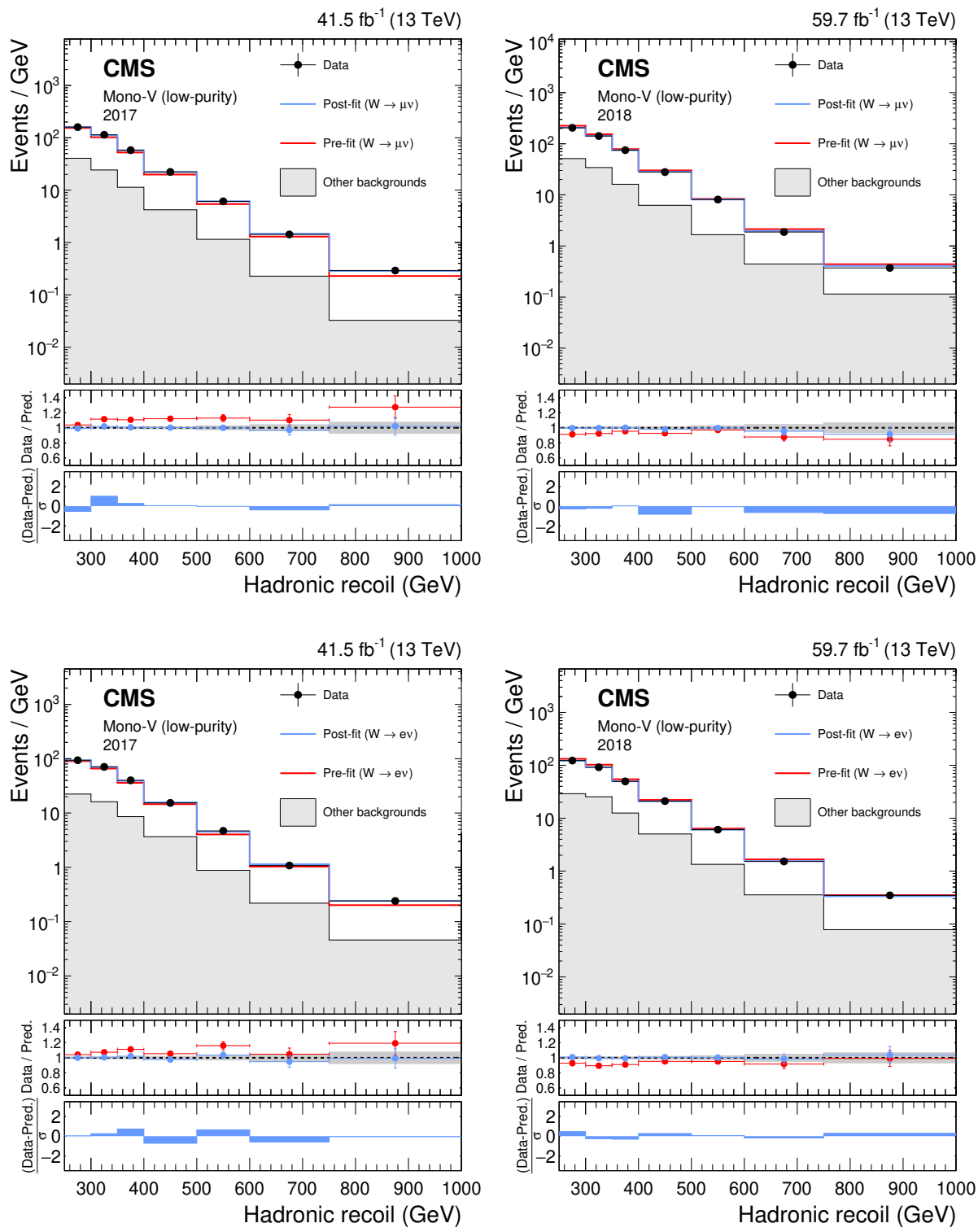

Figure 16. Hadronic recoil distributions in the single muon (upper), and single electron regions (lower) in the low-purity mono-V category. The "Other backgrounds" include top quark, diboson, and QCD multijet processes. 

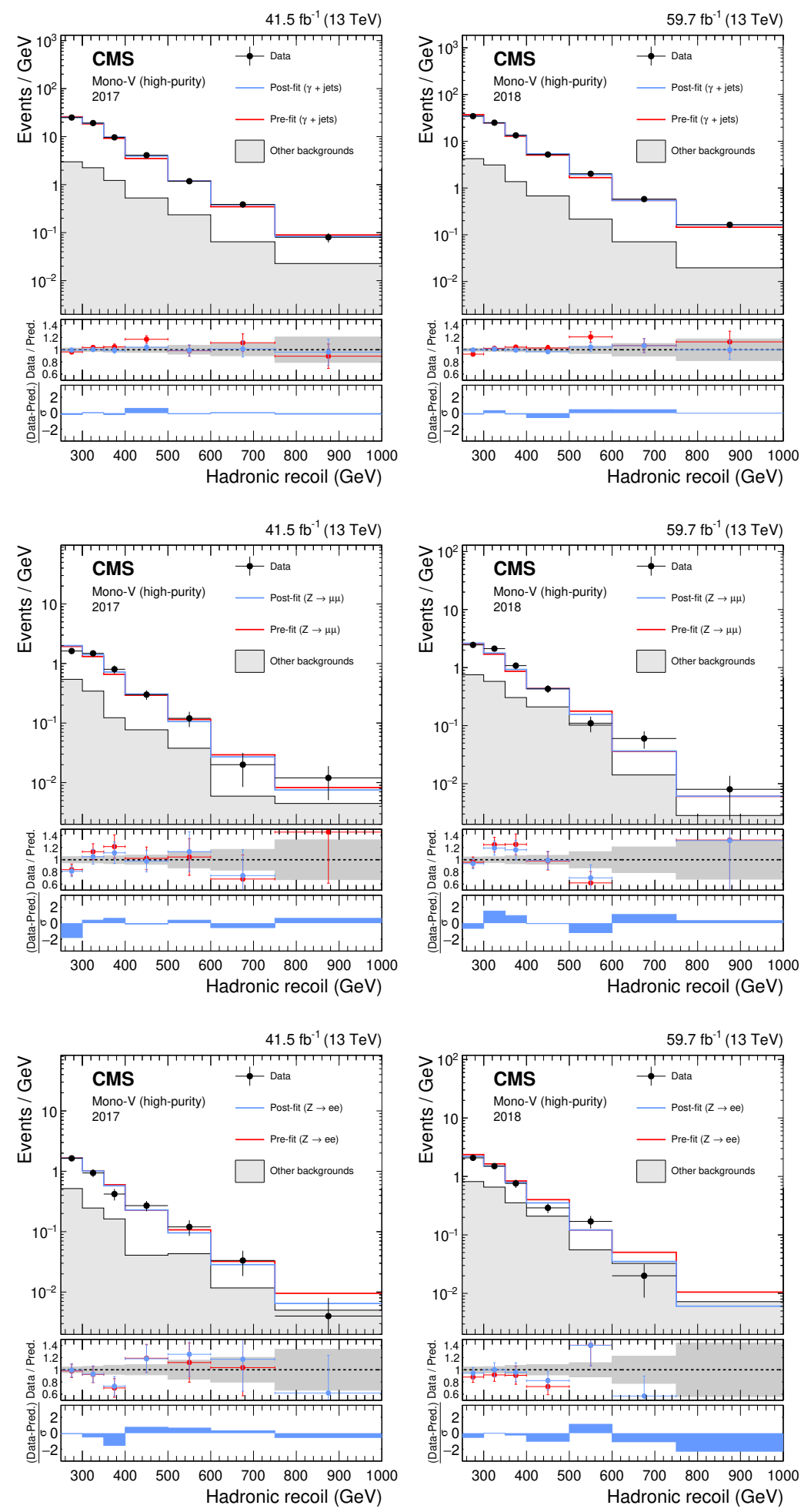

Figure 17. Hadronic recoil distributions in the photon (upper), dimuon (middle) and dielectron control regions (lower) in the high-purity mono-V category. The "Other backgrounds" include QCD multijet production (photon control region), and top quark, diboson, and $\mathrm{W}+$ jets processes (dimuon and dielectron control regions). 

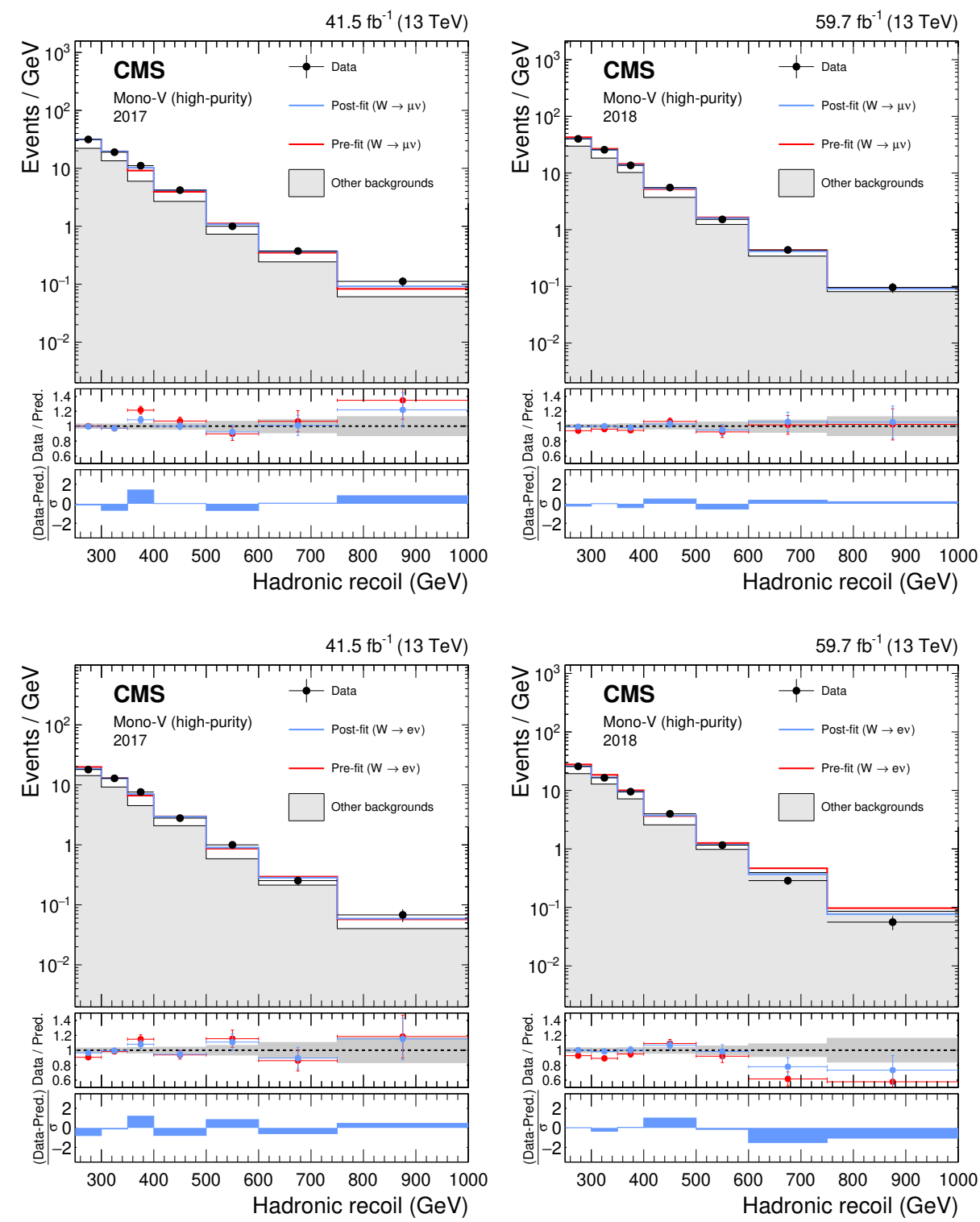

Figure 18. Hadronic recoil distributions in the single muon (upper), and single electron regions (lower) in the high-purity mono-V category. The "Other backgrounds" include top quark, diboson, and QCD multijet processes. 


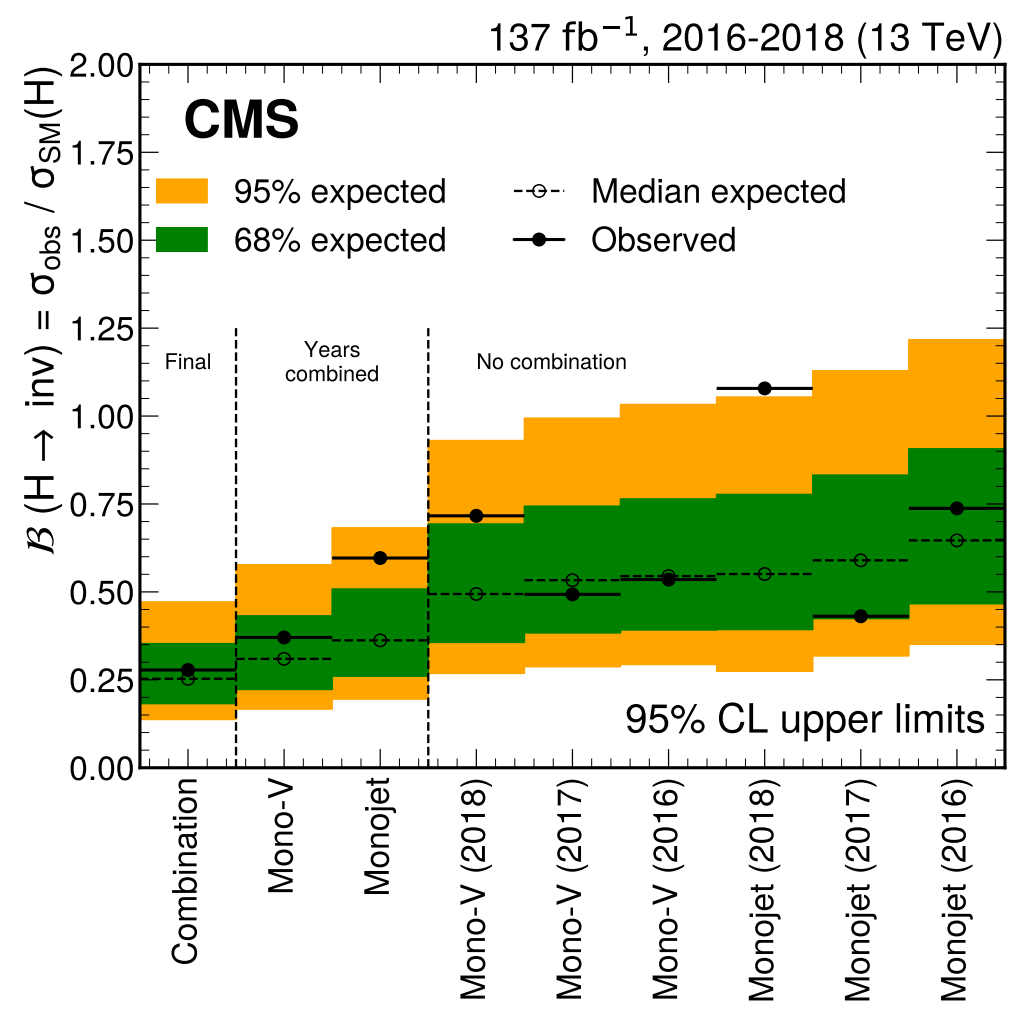

Figure 19. Exclusion limits at 95\% CL on the branching fraction of the Higgs boson to invisible particles. The result is shown separately for the monojet and mono- $\mathrm{V}$ categories in each data taking year, as well as their combination. The final combined limit is $27.8 \%$ (25.3\% expected).

\section{A.3 Exclusion in the Higgs portal interpretation split by data taking year}

The constraints placed on decays of the Higgs boson to invisible particles in each data taking year and category are summarized in figure 19. For each individual category and year, separate ML fits are performed, leading to independent best fit values of the nuisance parameters, as well as the signal strength. 

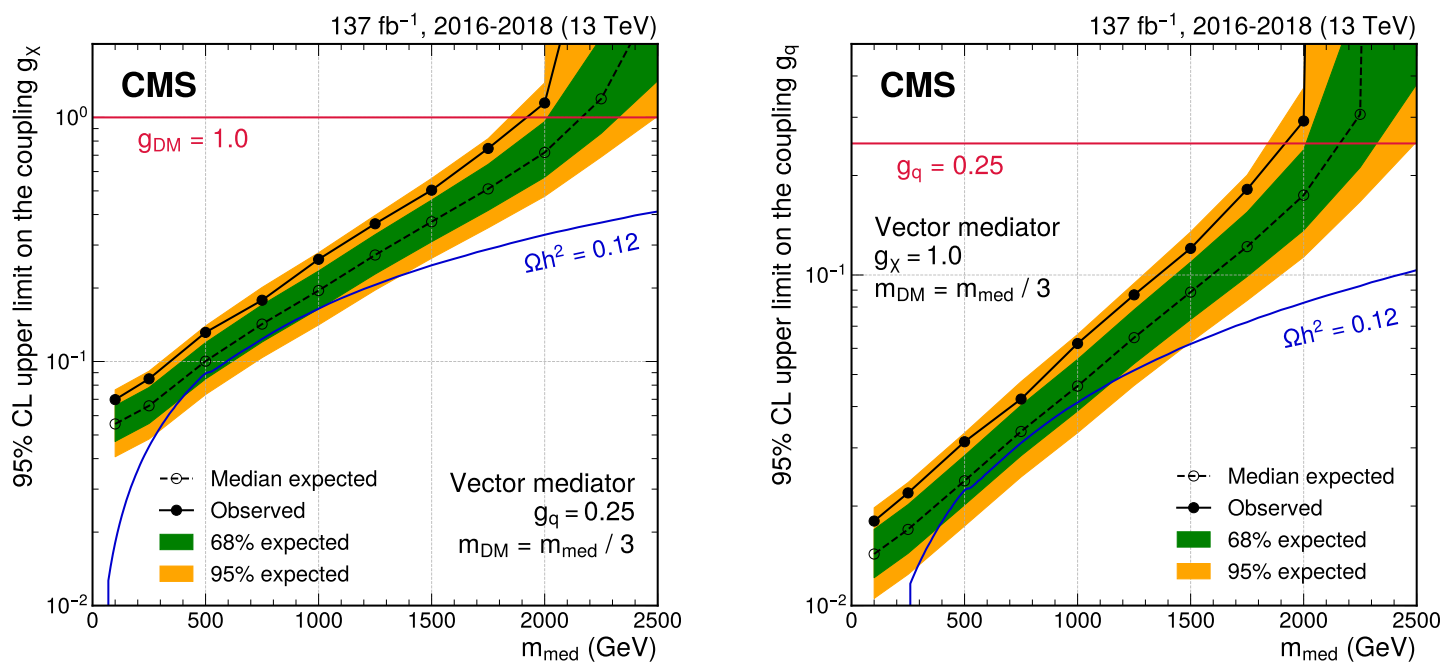

Figure 20. Exclusion limits at $95 \% \mathrm{CL}$ on the couplings $g_{\chi}$ (left) and $g_{\mathrm{q}}$ (right) for a vector mediator. In each panel, the result is shown as a function of the mediator mass $m_{\text {med }}$, and the mass of the DM candidate is fixed to $m_{\mathrm{DM}}=m_{\mathrm{med}} / 3$. In either case, only one coupling is varied, and the respective other coupling is fixed at its default value $\left(g_{\mathrm{q}}=0.25, g_{\chi}=1.0\right)$. The blue solid line indicates the parameter combinations for which the simplified model reproduces the observed $\mathrm{DM}$ relic density. Around $m_{\mathrm{DM}} \approx m_{\mathrm{top}}$, corresponding to $m_{\text {med }} \approx 500 \mathrm{GeV}, \mathrm{DM}$ annihilation into top quarks becomes possible, leading to a shift in the relic density.

\section{A.4 Coupling limits in a simplified DM model with a vector mediator}

Coupling limits for a vector mediator are derived in the same manner as the result for an axial mediator shown in figure 8. The result is shown in figure 20. 


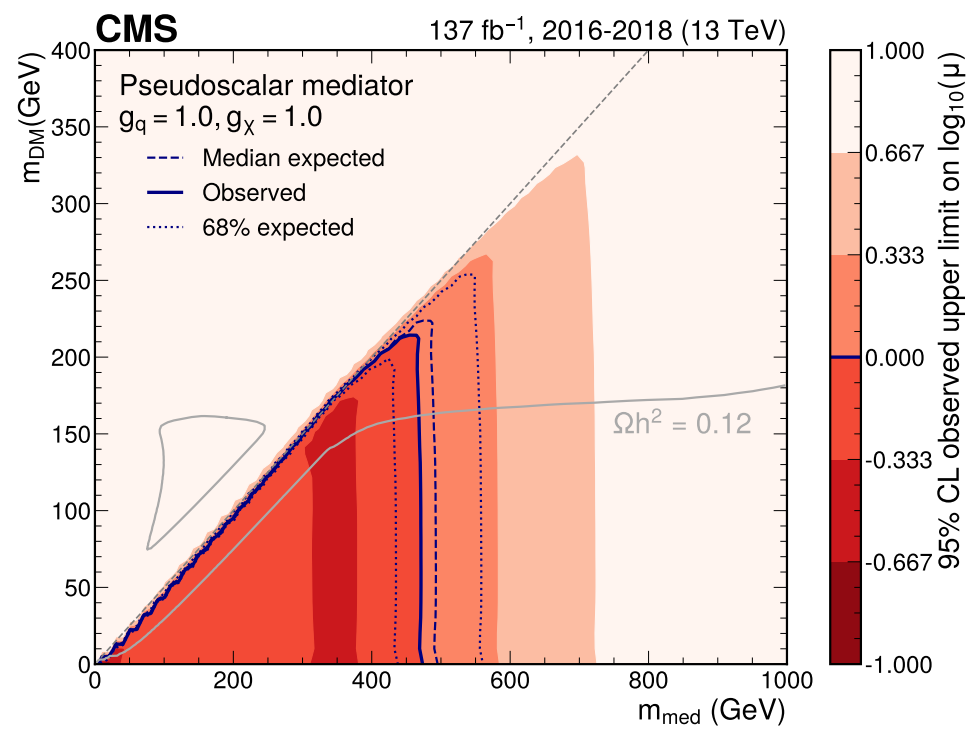

Figure 21. Exclusion limits at $95 \%$ CL on the signal strength $\mu=\sigma / \sigma_{\text {theo }}$ in the $m_{\text {med }}-m_{\text {DM }}$ plane for coupling values of $g_{\mathrm{q}}=g_{\chi}=1.0$ and a pseudoscalar mediator. The blue solid line indicates the observed exclusion boundary $\mu=1$. The blue dashed and dotted lines represent expected exclusion and the $68 \%$ CL interval of the expected boundary, respectively. Parameter combinations with larger values of $\mu$ (indicated by a darker shade in the color scale) are excluded. The gray dashed line indicates the diagonal $m_{\text {med }}=2 m_{\mathrm{DM}}$, above which only off-shell mediator production contributes to the jet $+p_{\mathrm{T}}^{\text {miss }}$ final state. The gray solid lines represent parameter combinations for which the simplified model reproduces the observed DM relic density in the universe under the assumption of a thermal freeze-out mechanism $[64,87]$.

\section{A.5 Two-dimensional exclusion in the simplified DM model with pseudoscalar mediator}

The exclusion limits in the $m_{\text {med }}-m_{\text {DM }}$ plane for the simplified model with a pseudoscalar mediator are shown figure 21. 


\begin{tabular}{rrr}
\hline$d$ & $\begin{array}{r}\text { Lower limit on } \\
\text { Expected }\end{array}$ & $\begin{array}{r}M_{\mathrm{D}}(\mathrm{TeV}) \\
\text { Observed }\end{array}$ \\
\hline 2 & 12.2 & 10.7 \\
3 & 9.0 & 8.0 \\
4 & 7.4 & 6.8 \\
5 & 6.6 & 6.0 \\
6 & 5.9 & 5.5 \\
7 & 5.6 & 5.2 \\
\hline
\end{tabular}

Table 3. Lower limits at 95\% CL on the fundamental Planck mass $M_{\mathrm{D}}$ in $\mathrm{TeV}$ as functions of the number of extra dimensions $d$.

\section{A.6 Table of exclusion limits in the ADD model}

The lower limits on the fundamental Planck mass $M_{\mathrm{D}}$ as a function of the number of extra dimensions are shown in table 3. 

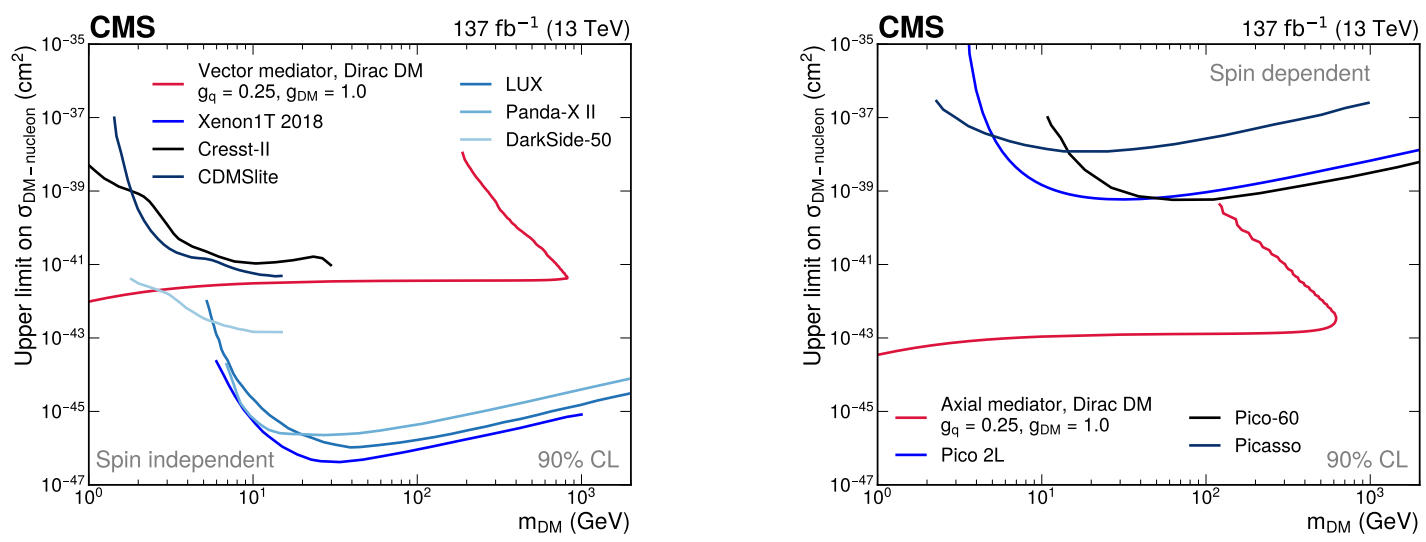

Figure 22. Comparison of the simplified model constraints from this search (red line) to results from direct-detection experiments (blue lines). The comparison is shown separately for the vector (left) and axial-vector (right) mediators, which translate into spin-independent and spindependent DM-nucleon couplings, respectively. In the case of spin-independent couplings, results from CRESST-II [90], CDMSlite [91], LUX [92], DarkSide-50 [93], XENON1T [94], and Panda-X II [95] are shown for comparison. For spin-dependent couplings, PICO-2L [96], PICASSO [97], and PICO-60 [98] limits are displayed.

\section{B Supplemental material}

\section{B.1 Comparison with direct-detection experiments}

The constraints placed on the $s$-channel simplified models imply bounds on the interaction cross section between DM candidates and nuclei. The fixed-coupling exclusion curves in the $m_{\text {med }}-m_{\text {DM }}$ plane are translated point-by-point using the formulae described in ref. [89], which depend on the coupling choices $g_{\mathrm{q}}=0.25$ and $g_{\chi}=1.0$ and on the specific signal model. The resulting curves in the $m_{\mathrm{DM}^{-}} \sigma_{\mathrm{DM} \text {-nucleon }}$ plane are compared to the results from direct-detection (DD) experiments in figure 22. Qualitatively, the results from this search depend on $m_{\mathrm{DM}}$ only weakly (as long as $m_{\mathrm{DM}}<m_{\text {med }} / 2$ ), leading to stringent constraints also at low values of $m_{\mathrm{DM}}$. The sensitivity of most $\mathrm{DD}$ experiments is limited in this regime as the small value of $m_{\mathrm{DM}}$ translates into a reduced signal-to-noise ratio relative to the case of more massive DM. Depending on the mediator type, the resulting couplings between DM particles and nuclei are either spin dependent (axial-vector) or independent (vector). In the spin-dependent case, the sensitivity of DD experiments is limited relative to collider searches as the DM-nucleus scattering is no longer coherent. 

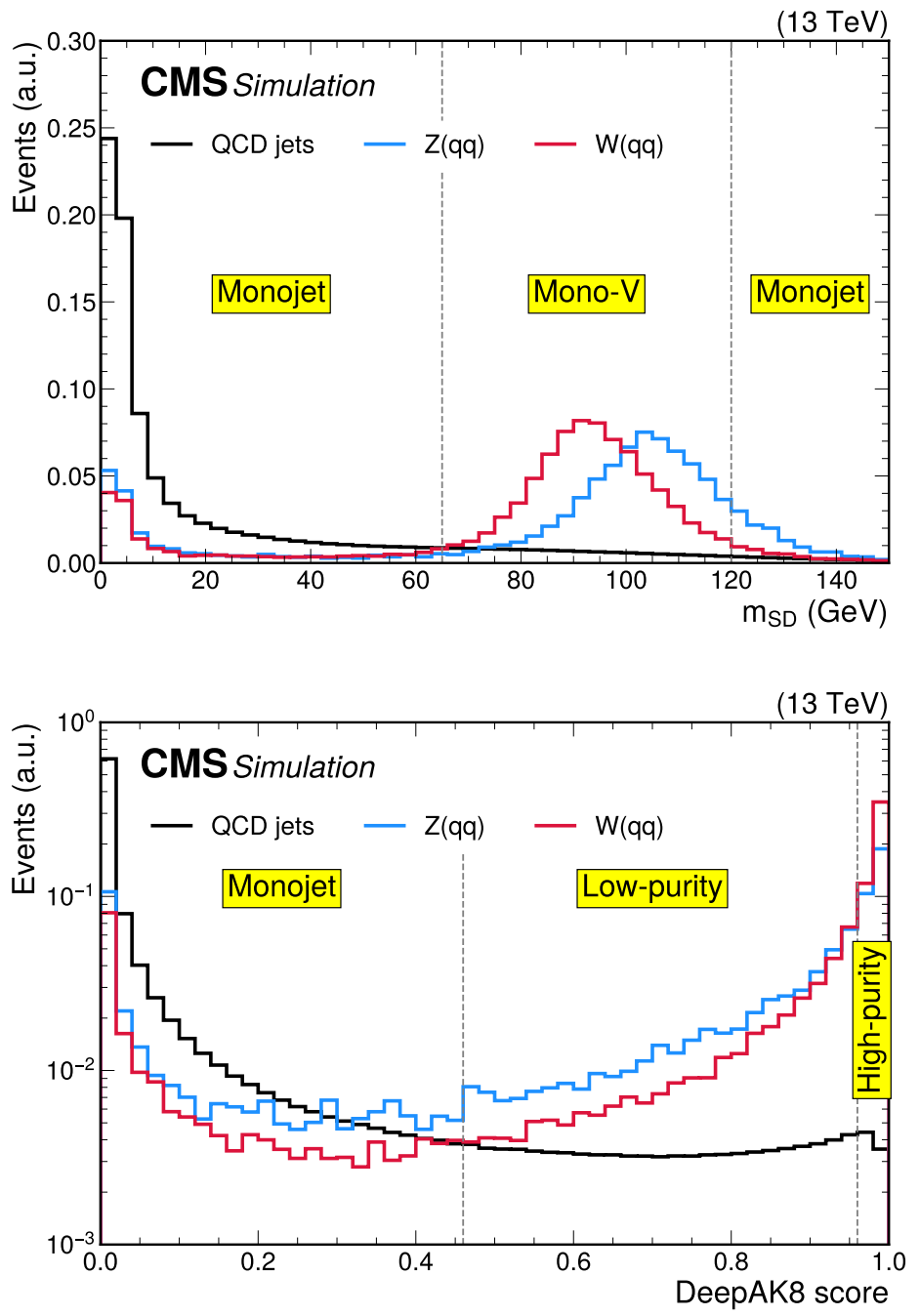

Figure 23. Distributions of the variables used for the identification of $\mathrm{V} \rightarrow \mathrm{qq}$ candidate jets. The upper and lower panels show the SD-corrected jet mass and the DEEPAK8 classifier value, respectively. In each panel, the distributions are shown for the $\mathrm{Z} \rightarrow \boldsymbol{v} v$ background, as well as the $\mathrm{WH}(\mathrm{inv}$.$) and \mathrm{ZH}(\mathrm{inv}$.$) signals. The distributions are shown after applying the mono-V signal$ region selection, with the exception of the requirements on the two variables shown here. Vertical dashed lines indicate the acceptance boundaries of different regions.

\section{B.2 Distributions of jet tagging variables}

The identification of the $\mathrm{V} \rightarrow \mathrm{qq}$ candidate large-radius jets relies on the SD-corrected mass of a given jet, as well as on the classifier score from the DEEPAK8 neural network. The ability of these quantities to separate genuine $\mathrm{V} \rightarrow$ qq candidates from background with jets originating from QCD radiation is demonstrated in figure 23. 


\section{B.3 Large-radius jet tagging efficiencies for reinterpretation}

To aid reinterpretation, the efficiency is calculated for the combination of the SD mass and DEePAK8 tagging requirements. The efficiency is calculated in simulated events passing the full signal mono- $\mathrm{V}$ region selections, with the exception of the requirements on $m_{\mathrm{SD}}$ and the tagging score. Correction factors accounting for the differences between data and simulation are included. The efficiencies are shown in figure 24 .

Efficiencies are provided for the low- and high-purity tagging requirements. We note that for the low-purity tagger, the overlap removal with the high-purity category is already done.

The efficiency is calculated separately for the AK8 jets matching a generator-level Z boson, W boson, or not matching either ("QCD jet"). A jet is considered to be matching a boson if their angular separation $\Delta R=\sqrt{(\Delta \eta)^{2}+(\Delta \phi)^{2}}$ is less than 0.8. In order to apply the efficiencies to simulated events, one should first apply all other selection criteria, except for the ones based on the jet mass or other substructure variables. Depending on the matching status of the jet, the respective efficiency evaluated at the $p_{\mathrm{T}}$ of the jet should be then applied as an event weight. 

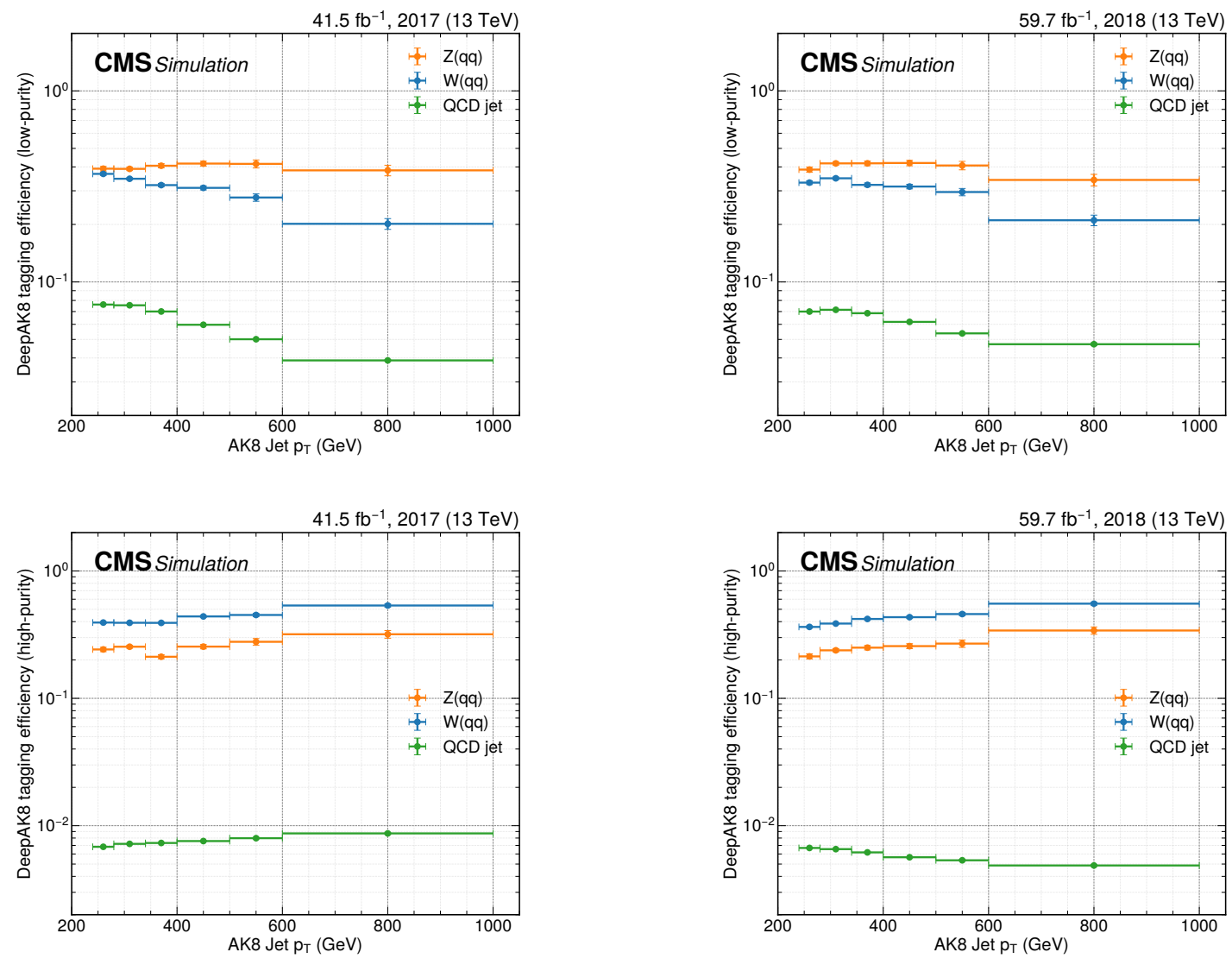

Figure 24. Large-radius jet tagging efficiencies for use in reinterpretation of the results. The efficiencies are shown separately for the low- and high-purity selections in the upper and lower panels, respectively, and for the 2017 (left) and 2018 (right) data taking periods. The efficiencies include the effect of the DEEPAK8 tagger, as well as the SD-corrected mass requirement. In each panel, individual curves represent the efficiency for different types of jets, based on whether the jets are matched to a generator-level W boson, Z boson, or neither ("QCD jet"). Simulation-to-data corrections are included. 

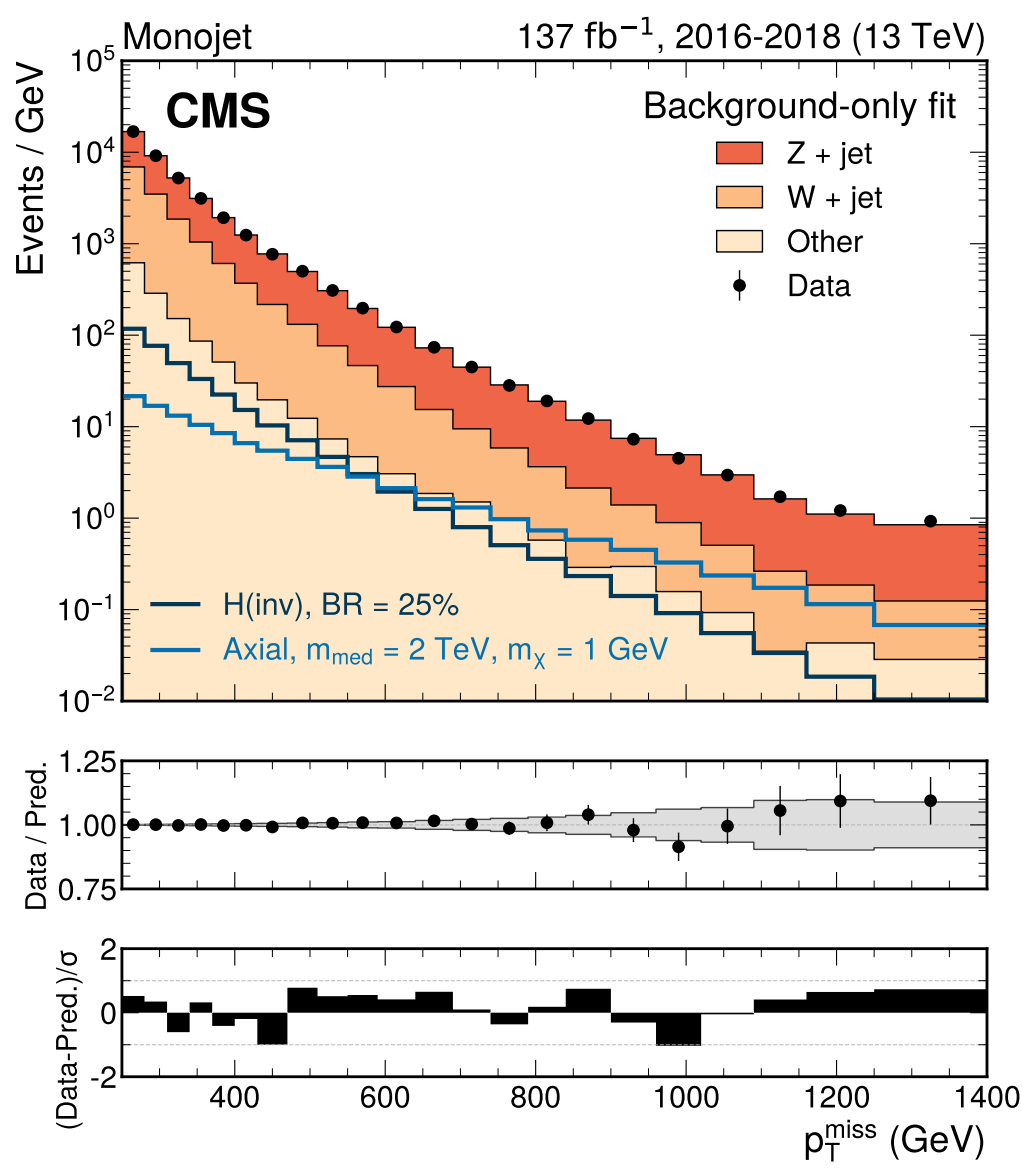

Figure 25. Distribution of $p_{\mathrm{T}}^{\text {miss }}$ in the monojet category. The distribution is shown including the contributions from all data taking years. It does not directly represent the input to the statistical method, which instead relies on distributions separated by data taking year. The background estimate is obtained from the background-only fit to all data taking periods, regions, and categories, including mono-V. The total uncertainty in the background estimate, shown as a gray band in the middle panel, takes into account all relevant correlations. The signal templates from the Higgs portal and axial-vector mediator hypotheses are overlaid (solid lines). In both cases, contributions from all production modes are taken into account.

\section{B.4 Monojet $p_{\mathrm{T}}^{\text {miss }}$ distribution for the full data set}

In the statistical analysis described in this paper, data from different data taking periods are sorted into separate bins. In figure 25 , the total $p_{\mathrm{T}}^{\text {miss }}$ distribution for all data taking years is shown, which is the bin-by-bin sum of the distributions in the individual years. 


\section{B.5 Analysis implementation in MadAnalysis}

The MADANALYsis package is a framework for the reinterpretation of existing analyses in terms of arbitrary new physics models [27]. The framework provides the infrastructure for the implementation of event selections that can be run over simulated signal events. Once an implementation is available, it is indexed in a public database that allows users to automatically download and execute it [28].

In order to promote this analysis for reinterpretation, we implement the selection for the monojet category of this analysis in MADANALYSIS. A total of 66 analysis regions are defined, with each of the regions representing one recoil bin in one data taking year. The selections applied for the 2016 and 2017 data sets are identical, and additional criteria are applied to the 2018 data set, where mitigation requirements are used because of a localized problem in the hadron calorimeter.

In order to validate the implementation, generator-level information from the simulated signal samples is fed into the DELPHES framework, which performs fast parameterized event simulation [99]. The MAdAnalysis implementation is then run based on the DeLPhes output, and the final yields per signal region bin are compared to the signal prediction obtained from the CMS analysis framework.

The comparison is made using signal samples for the ADD interpretation, which are generated using PYTHIA, and are therefore relatively easy to reproduce. The resulting comparison of the final signal templates is shown in figure 26 for a representative choice of parameter points. It is found that the DELPhES/MADANALYSIS-based result agrees with the CMS result to better than $20 \%$ in every bin. In most bins, the agreement is at the $10 \%$ or better level. While only a few parameter points are shown here, it has been verified that the agreement is similar for the full range of parameters. The level of agreement observed here is sufficiently good to enable reliable reinterpretation. 

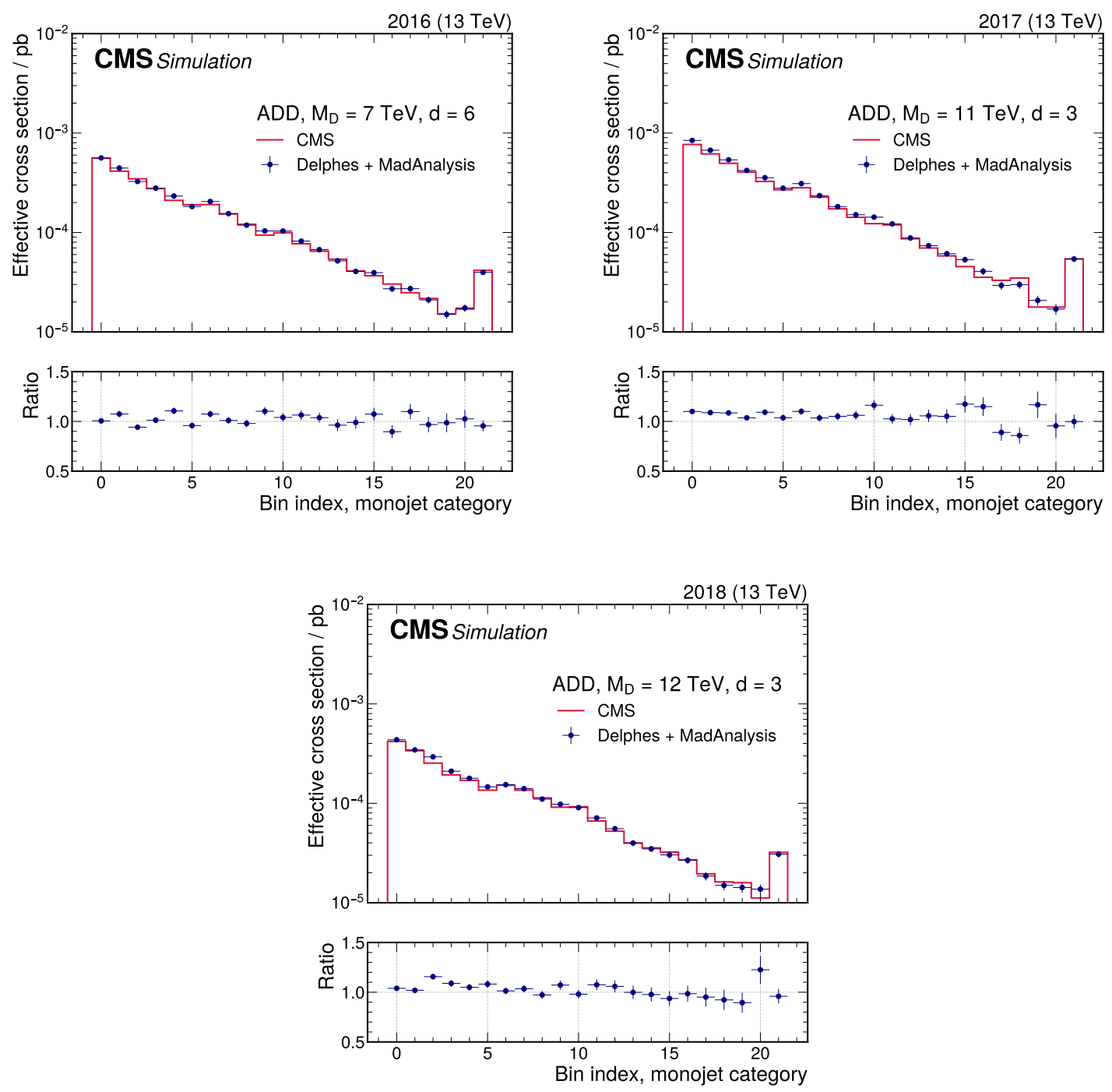

Figure 26. Comparison of the signal templates derived with Delphes and MadAnalysis (dark blue points) and the CMS analysis work flow (red solid line). The panels show three example parameter points for the ADD interpretation, and showcase the selection procedure for different years (2016 in the upper left, 2017 in the upper right, and 2018 in the lower panels). The rightmost bin includes the overflow. In all cases, the average agreement is observed to be better than $10 \%$, with maximum deviations up to $20 \%$ in single bins. 


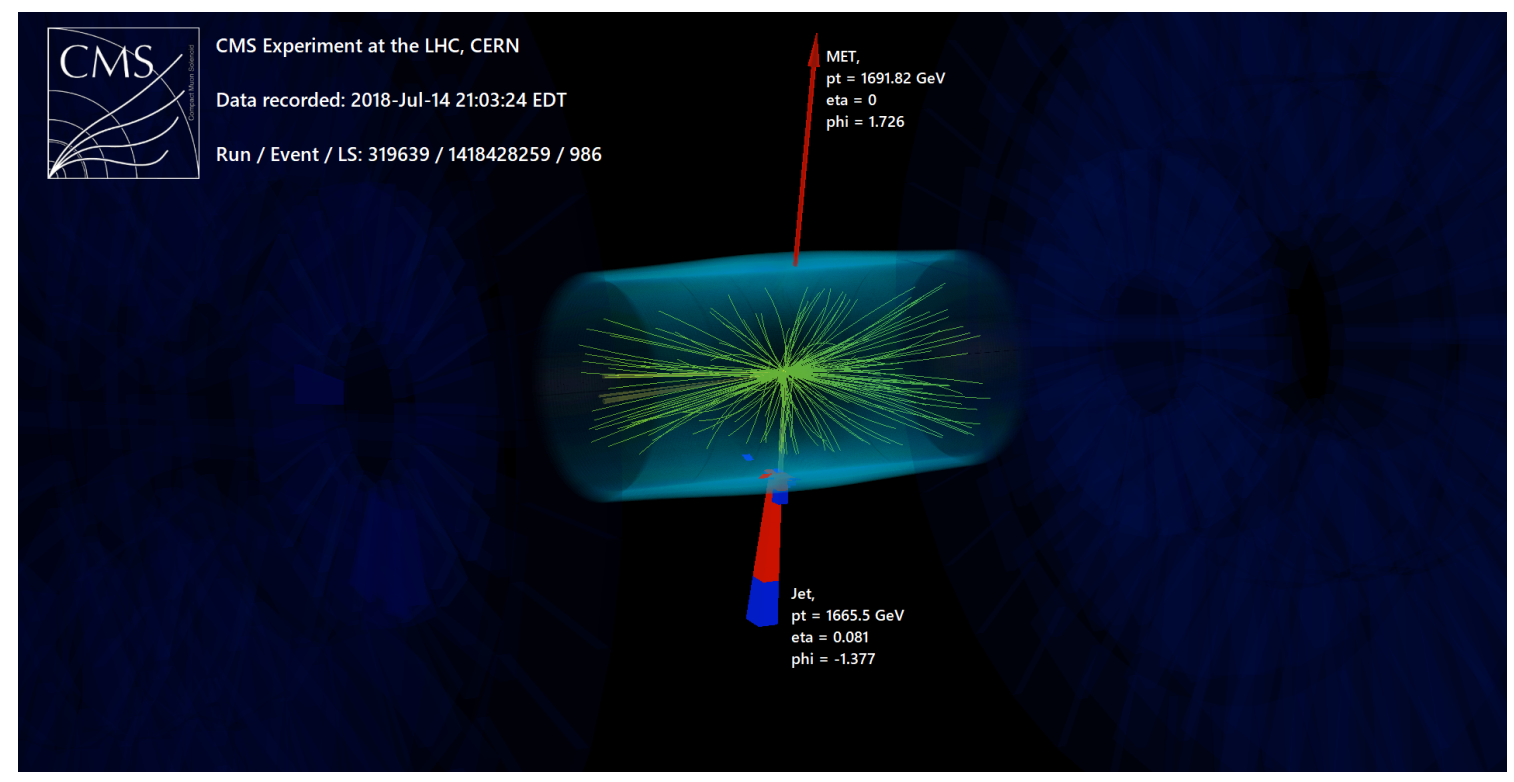

Figure 27. Display of a representative high- $p_{\mathrm{T}}^{\text {miss }}$ event from the monojet category in the 2018 data set. In this event, a single high- $p_{\mathrm{T}}$ jet (calorimeter deposits indicated by the red and blue towers) recoils against large $p_{\mathrm{T}}^{\text {miss }}$ (indicated by the red arrow).

\section{B.6 Event display}

A graphical rendering of an observed high- $p_{\mathrm{T}}^{\text {miss }}$ collision event in the CMS detector is shown in figure 27.

Open Access. This article is distributed under the terms of the Creative Commons Attribution License (CC-BY 4.0), which permits any use, distribution and reproduction in any medium, provided the original author(s) and source are credited.

\section{References}

[1] G. Bertone, D. Hooper and J. Silk, Particle dark matter: evidence, candidates and constraints, Phys. Rept. 405 (2005) 279 [hep-ph/0404175] [INSPIRE].

[2] S. Kanemura, S. Matsumoto, T. Nabeshima and N. Okada, Can WIMP dark matter overcome the nightmare scenario?, Phys. Rev. D 82 (2010) 055026 [arXiv:1005.5651] [INSPIRE].

[3] O. Lebedev, H.M. Lee and Y. Mambrini, Vector Higgs-portal dark matter and the invisible Higgs, Phys. Lett. B 707 (2012) 570 [arXiv:1111.4482] [InSPIRE].

[4] A. Djouadi, O. Lebedev, Y. Mambrini and J. Quevillon, Implications of LHC searches for Higgs-portal dark matter, Phys. Lett. B 709 (2012) 65 [arXiv:1112.3299] [INSPIRE].

[5] ATLAS collaboration, Observation of a new particle in the search for the Standard Model Higgs boson with the ATLAS detector at the LHC, Phys. Lett. B 716 (2012) 1 [arXiv: 1207.7214] [INSPIRE].

[6] CMS collaboration, Observation of a new boson at a mass of $125 \mathrm{GeV}$ with the CMS experiment at the LHC, Phys. Lett. B 716 (2012) 30 [arXiv:1207.7235] [INSPIRE]. 
[7] CMS collaboration, Observation of a new boson with mass near $125 \mathrm{GeV}$ in pp collisions at $\sqrt{s}=7$ and $8 \mathrm{TeV}, \mathrm{JHEP} 06$ (2013) 081 [arXiv: 1303.4571] [INSPIRE].

[8] ATLAS collaboration, Combination of searches for invisible Higgs boson decays with the ATLAS experiment, Phys. Rev. Lett. 122 (2019) 231801 [arXiv: 1904.05105] [INSPIRE].

[9] CMS collaboration, Search for invisible decays of a Higgs boson produced through vector boson fusion in proton-proton collisions at $\sqrt{s}=13$ TeV, Phys. Lett. B 793 (2019) 520 [arXiv: 1809.05937] [INSPIRE].

[10] D. Abercrombie et al., Dark matter benchmark models for early LHC run-2 searches: report of the ATLAS/CMS dark matter forum, Phys. Dark Univ. 27 (2020) 100371 [arXiv: 1507.00966] [INSPIRE].

[11] Y. Bai and J. Berger, Fermion portal dark matter, JHEP 11 (2013) 171 [arXiv:1308.0612] [INSPIRE].

[12] A. DiFranzo, K.I. Nagao, A. Rajaraman and T.M.P. Tait, Simplified models for dark matter interacting with quarks, JHEP 11 (2013) 014 [Erratum ibid. 01 (2014) 162] [arXiv:1308.2679] [INSPIRE].

[13] N. Arkani-Hamed, S. Dimopoulos and G.R. Dvali, The hierarchy problem and new dimensions at a millimeter, Phys. Lett. B 429 (1998) 263 [hep-ph/9803315] [InSPIRE].

[14] N. Arkani-Hamed, S. Dimopoulos and G.R. Dvali, Phenomenology, astrophysics and cosmology of theories with submillimeter dimensions and TeV scale quantum gravity, Phys. Rev. D 59 (1999) 086004 [hep-ph/9807344] [INSPIRE].

[15] CMS collaboration, Identification of heavy, energetic, hadronically decaying particles using machine-learning techniques, 2020 JINST 15 P06005 [arXiv:2004.08262] [INSPIRE].

[16] J.C. Pati and A. Salam, Unified lepton-hadron symmetry and a gauge theory of the basic interactions, Phys. Rev. D 8 (1973) 1240 [INSPIRE].

[17] J.C. Pati and A. Salam, Lepton number as the fourth color, Phys. Rev. D 10 (1974) 275 [Erratum ibid. 11 (1975) 703] [INSPIRE].

[18] H. Georgi and S.L. Glashow, Unity of all elementary particle forces, Phys. Rev. Lett. 32 (1974) 438 [INSPIRE].

[19] B. Diaz, M. Schmaltz and Y.-M. Zhong, The leptoquark hunter's guide: pair production, JHEP 10 (2017) 097 [arXiv:1706.05033] [INSPIRE].

[20] J.L. Hewett and S. Pakvasa, Leptoquark production in hadron colliders, Phys. Rev. D 37 (1988) 3165 [INSPIRE].

[21] O.J.P. Eboli and A.V. Olinto, Composite leptoquarks in hadronic colliders, Phys. Rev. D 38 (1988) 3461 [INSPIRE].

[22] CMS collaboration, Search for new physics in final states with an energetic jet or a hadronically decaying $W$ or $Z$ boson and transverse momentum imbalance at $\sqrt{s}=13$ TeV, Phys. Rev. D 97 (2018) 092005 [arXiv:1712.02345] [InSPIRE].

[23] ATLAS collaboration, Search for dark matter in events with a hadronically decaying vector boson and missing transverse momentum in pp collisions at $\sqrt{s}=13 \mathrm{TeV}$ with the ATLAS detector, JHEP 10 (2018) 180 [arXiv:1807.11471] [INSPIRE]. 
[24] ATLAS collaboration, Search for new phenomena in events with an energetic jet and missing transverse momentum in pp collisions at $\sqrt{s}=13 \mathrm{TeV}$ with the ATLAS detector, Phys. Rev. D 103 (2021) 112006 [arXiv:2102.10874] [INSPIRE].

[25] J. Thaler and K. Van Tilburg, Identifying boosted objects with $N$-subjettiness, JHEP 03 (2011) 015 [arXiv: 1011.2268] [INSPIRE].

[26] CMS collaboration, HEP data record for this analysis, CMS-EXO-20-004, (2021).

[27] E. Conte, B. Dumont, B. Fuks and C. Wymant, Designing and recasting LHC analyses with MadAnalysis 5, Eur. Phys. J. C $\mathbf{7 4}$ (2014) 3103 [arXiv:1405.3982] [INSPIRE].

[28] B. Dumont et al., Toward a public analysis database for LHC new physics searches using MadAnalysis 5, Eur. Phys. J. C 75 (2015) 56 [arXiv:1407.3278] [InSPIRE].

[29] CMS collaboration, Search for new particles in events with energetic jets and large missing transverse momentum in proton-proton collisions at $\sqrt{s}=13 \mathrm{TeV}$, Tech. Rep. CMS-EXO-20-004, CERN, Geneva, Switzerland (2021).

[30] CMS collaboration, Performance of the CMS level-1 trigger in proton-proton collisions at $\sqrt{s}=13 \mathrm{TeV}, 2020$ JINST 15 P10017 [arXiv: 2006. 10165] [inSPIRE].

[31] CMS collaboration, The CMS trigger system, 2017 JINST 12 P01020 [arXiv:1609.02366] [INSPIRE].

[32] CMS collaboration, The CMS experiment at the CERN LHC, 2008 JINST 3 S08004 [INSPIRE].

[33] M. Cacciari, G.P. Salam and G. Soyez, The anti- $k_{t}$ jet clustering algorithm, JHEP 04 (2008) 063 [arXiv: 0802.1189] [INSPIRE].

[34] M. Cacciari, G.P. Salam and G. Soyez, FastJet user manual, Eur. Phys. J. C 72 (2012) 1896 [arXiv: 1111.6097] [INSPIRE].

[35] CMS collaboration, Particle-flow reconstruction and global event description with the CMS detector, 2017 JINST 12 P10003 [arXiv:1706.04965] [INSPIRE].

[36] CMS collaboration, Jet energy scale and resolution in the CMS experiment in pp collisions at $8 \mathrm{TeV}, 2017$ JINST 12 P02014 [arXiv: 1607. 03663] [INSPIRE].

[37] CMS collaboration, Jet algorithms performance in $13 \mathrm{TeV}$ data, Tech. Rep. CMS-PAS-JME-16-003, CERN, Geneva, Switzerland (2017).

[38] CMS collaboration, Performance of missing transverse momentum reconstruction in proton-proton collisions at $\sqrt{s}=13 \mathrm{TeV}$ using the CMS detector, 2019 JINST $14 \mathrm{P} 07004$ [arXiv: 1903.06078] [INSPIRE].

[39] D. Bertolini, P. Harris, M. Low and N. Tran, Pileup per particle identification, JHEP 10 (2014) 059 [arXiv: 1407.6013] [INSPIRE].

[40] M. Dasgupta, A. Fregoso, S. Marzani and G.P. Salam, Towards an understanding of jet substructure, JHEP 09 (2013) 029 [arXiv:1307.0007] [INSPIRE].

[41] J.M. Butterworth, A.R. Davison, M. Rubin and G.P. Salam, Jet substructure as a new Higgs search channel at the LHC, Phys. Rev. Lett. 100 (2008) 242001 [arXiv:0802.2470] [INSPIRE].

[42] A.J. Larkoski, S. Marzani, G. Soyez and J. Thaler, Soft drop, JHEP 05 (2014) 146 [arXiv: 1402.2657] [INSPIRE]. 
[43] T. Sjöstrand et al., An introduction to PYTHIA 8.2, Comput. Phys. Commun. 191 (2015) 159 [arXiv: 1410.3012] [INSPIRE].

[44] CMS collaboration, Extraction and validation of a new set of CMS PYTHIA8 tunes from underlying-event measurements, Eur. Phys. J. C 80 (2020) 4 [arXiv:1903.12179] [InSPIRE].

[45] GEANT4 collaboration, GEANT4 - a simulation toolkit, Nucl. Instrum. Meth. A 506 (2003) 250 [INSPIRE].

[46] NNPDF collaboration, Parton distributions from high-precision collider data, Eur. Phys. J. C 77 (2017) 663 [arXiv: 1706.00428] [INSPIRE].

[47] J. Alwall et al., The automated computation of tree-level and next-to-leading order differential cross sections, and their matching to parton shower simulations, JHEP 07 (2014) 079 [arXiv: 1405.0301] [INSPIRE].

[48] R. Frederix and S. Frixione, Merging meets matching in MC@NLO, JHEP 12 (2012) 061 [arXiv:1209.6215] [INSPIRE].

[49] M. Czakon, P. Fiedler and A. Mitov, Total top-quark pair-production cross section at hadron colliders through $O\left(\alpha_{S}^{4}\right)$, Phys. Rev. Lett. 110 (2013) 252004 [arXiv:1303.6254] [INSPIRE].

[50] S. Alioli, P. Nason, C. Oleari and E. Re, NLO single-top production matched with shower in POWHEG: s- and t-channel contributions, JHEP 09 (2009) 111 [Erratum ibid. 02 (2010) 011] [arXiv: 0907.4076] [INSPIRE].

[51] E. Re, Single-top Wt-channel production matched with parton showers using the POWHEG method, Eur. Phys. J. C 71 (2011) 1547 [arXiv: 1009.2450] [INSPIRE].

[52] N. Kidonakis, Two-loop soft anomalous dimensions for single top quark associated production with $a W^{-}$or $H^{-}$, Phys. Rev. D 82 (2010) 054018 [arXiv: 1005.4451] [inSPIRE].

[53] M. Aliev, H. Lacker, U. Langenfeld, S. Moch, P. Uwer and M. Wiedermann, HATHOR: HAdronic Top and Heavy quarks crOss section calculatoR, Comput. Phys. Commun. 182 (2011) 1034 [arXiv: 1007.1327] [inSPIRE].

[54] P. Kant et al., HatHor for single top-quark production: updated predictions and uncertainty estimates for single top-quark production in hadronic collisions, Comput. Phys. Commun. 191 (2015) 74 [arXiv: 1406.4403] [INSPIRE].

[55] T. Gehrmann et al., $W^{+} W^{-}$production at hadron colliders in next to next to leading order QCD, Phys. Rev. Lett. 113 (2014) 212001 [arXiv:1408.5243] [INSPIRE].

[56] J.M. Campbell and R.K. Ellis, An update on vector boson pair production at hadron colliders, Phys. Rev. D 60 (1999) 113006 [hep-ph/9905386] [INSPIRE].

[57] E. Bagnaschi, G. Degrassi, P. Slavich and A. Vicini, Higgs production via gluon fusion in the POWHEG approach in the SM and in the MSSM, JHEP 02 (2012) 088 [arXiv:1111.2854] [INSPIRE].

[58] G. Luisoni, P. Nason, C. Oleari and F. Tramontano, $H W^{ \pm} / H Z+0$ and 1 jet at $N L O$ with the POWHEG BOX interfaced to GoSam and their merging within MiNLO, JHEP 10 (2013) 083 [arXiv: 1306.2542] [INSPIRE].

[59] P. Nason and C. Oleari, NLO Higgs boson production via vector-boson fusion matched with shower in POWHEG, JHEP 02 (2010) 037 [arXiv:0911.5299] [INSPIRE]. 
[60] LHC Higgs Cross Section Working Group collaboration, Handbook of LHC Higgs cross sections: 4. Deciphering the nature of the Higgs sector, arXiv:1610.07922 [INSPIRE].

[61] O. Mattelaer and E. Vryonidou, Dark matter production through loop-induced processes at the LHC: the s-channel mediator case, Eur. Phys. J. C 75 (2015) 436 [arXiv:1508.00564] [INSPIRE].

[62] M. Backović, M. Krämer, F. Maltoni, A. Martini, K. Mawatari and M. Pellen, Higher-order QCD predictions for dark matter production at the LHC in simplified models with s-channel mediators, Eur. Phys. J. C 75 (2015) 482 [arXiv: 1508.05327] [INSPIRE].

[63] M. Neubert, J. Wang and C. Zhang, Higher-order QCD predictions for dark matter production in mono-Z searches at the LHC, JHEP 02 (2016) 082 [arXiv:1509.05785] [INSPIRE].

[64] A. Albert et al., Recommendations of the LHC dark matter working group: comparing LHC searches for dark matter mediators in visible and invisible decay channels and calculations of the thermal relic density, Phys. Dark Univ. 26 (2019) 100377 [arXiv:1703.05703] [INSPIRE].

[65] C. Arina, B. Fuks and L. Mantani, A universal framework for $t$-channel dark matter models, Eur. Phys. J. C 80 (2020) 409 [arXiv:2001.05024] [inSPIRE].

[66] S. Ask, I.V. Akin, L. Benucci, A. De Roeck, M. Goebel and J. Haller, Real emission and virtual exchange of gravitons and unparticles in PYTHIA8, Comput. Phys. Commun. 181 (2010) 1593 [arXiv: 0912 .4233] [inSPIRE].

[67] CMS collaboration, Electron and photon reconstruction and identification with the CMS experiment at the CERN LHC, 2021 JINST 16 P05014 [arXiv:2012.06888] [INSPIRE].

[68] CMS collaboration, Performance of the CMS muon detector and muon reconstruction with proton-proton collisions at $\sqrt{s}=13 \mathrm{TeV}, 2018$ JINST $13 \mathrm{P} 06015$ [arXiv:1804.04528] [INSPIRE].

[69] CMS collaboration, Performance of reconstruction and identification of $\tau$ leptons decaying to hadrons and $\nu_{\tau}$ in pp collisions at $\sqrt{s}=13 \mathrm{TeV}, 2018$ JINST $13 \mathrm{P} 10005$ [arXiv: 1809.02816] [INSPIRE].

[70] CMS collaboration, Identification of heavy-flavour jets with the CMS detector in $p p$ collisions at $13 \mathrm{TeV}, 2018$ JINST 13 P05011 [arXiv:1712.07158] [INSPIRE].

[71] Particle Data Group collaboration, Review of particle physics, PTEP 2020 (2020) 083C01 [INSPIRE].

[72] CMS collaboration, Search for dark matter produced with an energetic jet or a hadronically decaying $W$ or $Z$ boson at $\sqrt{s}=13 \mathrm{TeV}$, JHEP 07 (2017) 014 [arXiv: 1703.01651] [INSPIRE].

[73] J.M. Lindert et al., Precise predictions for $V+$ jets dark matter backgrounds, Eur. Phys. J. C 77 (2017) 829 [arXiv: 1705.04664] [INSPIRE].

[74] J. Baglio, L.D. Ninh and M.M. Weber, Massive gauge boson pair production at the LHC: a next-to-leading order story, Phys. Rev. D 88 (2013) 113005 [Erratum ibid. 94 (2016) 099902] [arXiv: 1307.4331] [INSPIRE].

[75] A. Denner, S. Dittmaier, M. Hecht and C. Pasold, NLO QCD and electroweak corrections to $W+\gamma$ production with leptonic $W$-boson decays, JHEP 04 (2015) 018 [arXiv: 1412.7421] [INSPIRE]. 
[76] A. Denner, S. Dittmaier, M. Hecht and C. Pasold, $N L O Q C D$ and electroweak corrections to $Z+\gamma$ production with leptonic Z-boson decays, JHEP 02 (2016) 057 [arXiv:1510.08742] [INSPIRE].

[77] J. Butterworth et al., PDF4LHC recommendations for LHC run II, J. Phys. G 43 (2016) 023001 [arXiv: 1510.03865] [INSPIRE].

[78] S. Dulat et al., New parton distribution functions from a global analysis of quantum chromodynamics, Phys. Rev. D 93 (2016) 033006 [arXiv:1506.07443] [inSPIRE].

[79] L.A. Harland-Lang, A.D. Martin, P. Motylinski and R.S. Thorne, Parton distributions in the LHC era: MMHT 2014 PDFs, Eur. Phys. J. C 75 (2015) 204 [arXiv:1412.3989] [inSPIRE].

[80] NNPDF collaboration, Parton distributions for the LHC run II, JHEP 04 (2015) 040 [arXiv: 1410.8849] [INSPIRE].

[81] M. Czakon, D. Heymes and A. Mitov, High-precision differential predictions for top-quark pairs at the LHC, Phys. Rev. Lett. 116 (2016) 082003 [arXiv:1511.00549] [INSPIRE].

[82] A.L. Read, Presentation of search results: the $C L_{s}$ technique, J. Phys. G 28 (2002) 2693 [INSPIRE].

[83] T. Junk, Confidence level computation for combining searches with small statistics, Nucl. Instrum. Meth. A 434 (1999) 435 [hep-ex/9902006] [INSPIRE].

[84] G. Cowan, K. Cranmer, E. Gross and O. Vitells, Asymptotic formulae for likelihood-based tests of new physics, Eur. Phys. J. C 71 (2011) 1554 [Erratum ibid. 73 (2013) 2501] [arXiv: 1007.1727] [INSPIRE].

[85] ATLAS collaboration, Search for low-mass dijet resonances using trigger-level jets with the ATLAS detector in pp collisions at $\sqrt{s}=13$ TeV, Phys. Rev. Lett. 121 (2018) 081801 [arXiv: 1804.03496] [INSPIRE].

[86] CMS collaboration, Search for low mass vector resonances decaying into quark-antiquark pairs in proton-proton collisions at $\sqrt{s}=13 \mathrm{TeV}$, Phys. Rev. D 100 (2019) 112007 [arXiv: 1909.04114] [INSPIRE].

[87] Planck collaboration, Planck 2018 results. VI. Cosmological parameters, Astron. Astrophys. 641 (2020) A6 [Erratum ibid. 652 (2021) C4] [arXiv: 1807.06209] [INSPIRE].

[88] CMS collaboration, Searches for physics beyond the standard model with the $M_{T 2}$ variable in hadronic final states with and without disappearing tracks in proton-proton collisions at $\sqrt{s}=13 \mathrm{TeV}$, Eur. Phys. J. C 80 (2020) 3 [arXiv:1909.03460] [INSPIRE].

[89] A. Boveia et al., Recommendations on presenting LHC searches for missing transverse energy signals using simplified s-channel models of dark matter, Phys. Dark Univ. 27 (2020) 100365 [arXiv: 1603.04156] [INSPIRE].

[90] CRESST collaboration, Results on light dark matter particles with a low-threshold CRESST-II detector, Eur. Phys. J. C 76 (2016) 25 [arXiv:1509.01515] [InSPIRE].

[91] SUPERCDMS collaboration, New results from the search for low-mass weakly interacting massive particles with the CDMS low ionization threshold experiment, Phys. Rev. Lett. 116 (2016) 071301 [arXiv: 1509.02448] [INSPIRE].

[92] LUX collaboration, Results from a search for dark matter in the complete LUX exposure, Phys. Rev. Lett. 118 (2017) 021303 [arXiv: 1608.07648] [INSPIRE]. 
[93] DARKSide collaboration, Low-mass dark matter search with the DarkSide-50 experiment, Phys. Rev. Lett. 121 (2018) 081307 [arXiv: 1802.06994] [INSPIRE].

[94] XENON collaboration, Dark matter search results from a one ton-year exposure of XENON1T, Phys. Rev. Lett. 121 (2018) 111302 [arXiv:1805.12562] [INSPIRE].

[95] PandaX-II collaboration, Results of dark matter search using the full PandaX-II exposure, Chin. Phys. C 44 (2020) 125001 [arXiv: 2007.15469] [INSPIRE].

[96] PICO collaboration, Improved dark matter search results from PICO-2L run 2, Phys. Rev. D 93 (2016) 061101 [arXiv: 1601.03729] [INSPIRE].

[97] E. Behnke et al., Final results of the PICASSO dark matter search experiment, Astropart. Phys. 90 (2017) 85 [arXiv: 1611.01499] [InSPIRE].

[98] PICO collaboration, Dark matter search results from the complete exposure of the PICO-60 $C_{3} F_{8}$ bubble chamber, Phys. Rev. D 100 (2019) 022001 [arXiv: 1902.04031] [INSPIRE].

[99] DELPHES 3 collaboration, DELPHES 3, a modular framework for fast simulation of a generic collider experiment, JHEP 02 (2014) 057 [arXiv: 1307.6346] [INSPIRE]. 


\section{The CMS collaboration}

Yerevan Physics Institute, Yerevan, Armenia

A. Tumasyan

Institut für Hochenergiephysik, Wien, Austria

W. Adam, J.W. Andrejkovic, T. Bergauer, S. Chatterjee, M. Dragicevic, A. Escalante Del Valle, R. Frühwirth $^{1}$, M. Jeitler ${ }^{1}$, N. Krammer, L. Lechner, D. Liko, I. Mikulec, P. Paulitsch, F.M. Pitters, J. Schieck ${ }^{1}$, R. Schöfbeck, D. Schwarz, S. Templ, W. Waltenberger, C.-E. Wulz ${ }^{1}$

Institute for Nuclear Problems, Minsk, Belarus

V. Chekhovsky, A. Litomin, V. Makarenko

Universiteit Antwerpen, Antwerpen, Belgium

M.R. Darwish ${ }^{2}$, E.A. De Wolf, T. Janssen, T. Kello ${ }^{3}$, A. Lelek, H. Rejeb Sfar, P. Van Mechelen, S. Van Putte, N. Van Remortel

Vrije Universiteit Brussel, Brussel, Belgium

F. Blekman, E.S. Bols, J. D'Hondt, M. Delcourt, H. El Faham, S. Lowette, S. Moortgat, A. Morton, D. Müller, A.R. Sahasransu, S. Tavernier, W. Van Doninck, P. Van Mulders

Université Libre de Bruxelles, Bruxelles, Belgium

D. Beghin, B. Bilin, B. Clerbaux, G. De Lentdecker, L. Favart, A. Grebenyuk, A.K. Kalsi, K. Lee, M. Mahdavikhorrami, I. Makarenko, L. Moureaux, L. Pétré, A. Popov, N. Postiau, E. Starling, L. Thomas, M. Vanden Bemden, C. Vander Velde, P. Vanlaer, L. Wezenbeek

\section{Ghent University, Ghent, Belgium}

T. Cornelis, D. Dobur, J. Knolle, L. Lambrecht, G. Mestdach, M. Niedziela, C. Roskas, A. Samalan, K. Skovpen, M. Tytgat, B. Vermassen, M. Vit

Université Catholique de Louvain, Louvain-la-Neuve, Belgium

A. Benecke, A. Bethani, G. Bruno, F. Bury, C. Caputo, P. David, C. Delaere, I.S. Donertas, A. Giammanco, K. Jaffel, Sa. Jain, V. Lemaitre, K. Mondal, J. Prisciandaro, A. Taliercio, M. Teklishyn, T.T. Tran, P. Vischia, S. Wertz

Centro Brasileiro de Pesquisas Fisicas, Rio de Janeiro, Brazil

G.A. Alves, C. Hensel, A. Moraes

Universidade do Estado do Rio de Janeiro, Rio de Janeiro, Brazil

W.L. Aldá Júnior, M. Alves Gallo Pereira, M. Barroso Ferreira Filho, H. Brandao Malbouisson, W. Carvalho, J. Chinellato ${ }^{4}$, E.M. Da Costa, G.G. Da Silveira ${ }^{5}$, D. De Jesus Damiao, S. Fonseca De Souza, D. Matos Figueiredo, C. Mora Herrera, K. Mota Amarilo, L. Mundim, H. Nogima, P. Rebello Teles, A. Santoro, S.M. Silva Do Amaral, A. Sznajder, M. Thiel, F. Torres Da Silva De Araujo ${ }^{6}$, A. Vilela Pereira 
Universidade Estadual Paulista ${ }^{a}$, Universidade Federal do $\mathrm{ABC}^{b}$, São Paulo, Brazil

C.A. Bernardes ${ }^{a, a, 5}$, L. Calligaris ${ }^{a}$, T.R. Fernandez Perez Tomei ${ }^{a}$, E.M. Gregores ${ }^{a, b}$, D.S. Lemos ${ }^{a}$, P.G. Mercadante ${ }^{a, b}$, S.F. Novaes ${ }^{a}$, Sandra S. Padula ${ }^{a}$

Institute for Nuclear Research and Nuclear Energy, Bulgarian Academy of Sciences, Sofia, Bulgaria

A. Aleksandrov, G. Antchev, R. Hadjiiska, P. Iaydjiev, M. Misheva, M. Rodozov,

M. Shopova, G. Sultanov

University of Sofia, Sofia, Bulgaria

A. Dimitrov, T. Ivanov, L. Litov, B. Pavlov, P. Petkov, A. Petrov

Beihang University, Beijing, China

T. Cheng, T. Javaid ${ }^{7}$, M. Mittal, L. Yuan

Department of Physics, Tsinghua University, Beijing, China

M. Ahmad, G. Bauer, C. Dozen ${ }^{8}$, Z. Hu, J. Martins ${ }^{9}$, Y. Wang, K. Yi ${ }^{10,11}$

Institute of High Energy Physics, Beijing, China

E. Chapon, G.M. Chen ${ }^{7}$, H.S. Chen ${ }^{7}$, M. Chen, F. Iemmi, A. Kapoor, D. Leggat, H. Liao, Z.-A. Liu ${ }^{7}$, V. Milosevic, F. Monti, R. Sharma, J. Tao, J. Thomas-wilsker, J. Wang, H. Zhang, J. Zhao

State Key Laboratory of Nuclear Physics and Technology, Peking University, Beijing, China

A. Agapitos, Y. An, Y. Ban, C. Chen, A. Levin, Q. Li, X. Lyu, Y. Mao, S.J. Qian, D. Wang, Q. Wang, J. Xiao

\section{Sun Yat-Sen University, Guangzhou, China}

M. Lu, Z. You

Institute of Modern Physics and Key Laboratory of Nuclear Physics and Ionbeam Application (MOE) — Fudan University, Shanghai, China

X. $\mathrm{Gao}^{3}, \mathrm{H}$. Okawa

Zhejiang University, Hangzhou, China

Z. Lin, M. Xiao

Universidad de Los Andes, Bogota, Colombia

C. Avila, A. Cabrera, C. Florez, J. Fraga

Universidad de Antioquia, Medellin, Colombia

J. Mejia Guisao, F. Ramirez, J.D. Ruiz Alvarez, C.A. Salazar González

University of Split, Faculty of Electrical Engineering, Mechanical Engineering and Naval Architecture, Split, Croatia

D. Giljanovic, N. Godinovic, D. Lelas, I. Puljak 
University of Split, Faculty of Science, Split, Croatia

Z. Antunovic, M. Kovac, T. Sculac

Institute Rudjer Boskovic, Zagreb, Croatia

V. Brigljevic, D. Ferencek, D. Majumder, M. Roguljic, A. Starodumov ${ }^{12}$, T. Susa

University of Cyprus, Nicosia, Cyprus

A. Attikis, K. Christoforou, E. Erodotou, A. Ioannou, G. Kole, M. Kolosova, S. Konstantinou, J. Mousa, C. Nicolaou, F. Ptochos, P.A. Razis, H. Rykaczewski, H. Saka

Charles University, Prague, Czech Republic

M. Finger ${ }^{13}$, M. Finger Jr. ${ }^{13}$, A. Kveton

Escuela Politecnica Nacional, Quito, Ecuador

E. Ayala

Universidad San Francisco de Quito, Quito, Ecuador

E. Carrera Jarrin

Academy of Scientific Research and Technology of the Arab Republic of Egypt, Egyptian Network of High Energy Physics, Cairo, Egypt

S. Elgammal ${ }^{14}$, S. Khalil ${ }^{15}$

Center for High Energy Physics (CHEP-FU), Fayoum University, El-Fayoum, Egypt

M.A. Mahmoud, Y. Mohammed

National Institute of Chemical Physics and Biophysics, Tallinn, Estonia

S. Bhowmik, R.K. Dewanjee, K. Ehataht, M. Kadastik, S. Nandan, C. Nielsen, J. Pata, M. Raidal, L. Tani, C. Veelken

Department of Physics, University of Helsinki, Helsinki, Finland

P. Eerola, L. Forthomme, H. Kirschenmann, K. Osterberg, M. Voutilainen

Helsinki Institute of Physics, Helsinki, Finland

S. Bharthuar, E. Brücken, F. Garcia, J. Havukainen, M.S. Kim, R. Kinnunen, T. Lampén, K. Lassila-Perini, S. Lehti, T. Lindén, M. Lotti, L. Martikainen, M. Myllymäki, J. Ott, H. Siikonen, E. Tuominen, J. Tuominiemi

Lappeenranta University of Technology, Lappeenranta, Finland

P. Luukka, H. Petrow, T. Tuuva

IRFU, CEA, Université Paris-Saclay, Gif-sur-Yvette, France

C. Amendola, M. Besancon, F. Couderc, M. Dejardin, D. Denegri, J.L. Faure, F. Ferri, S. Ganjour, A. Givernaud, P. Gras, G. Hamel de Monchenault, P. Jarry, B. Lenzi, E. Locci, J. Malcles, J. Rander, A. Rosowsky, M.Ö. Sahin, A. Savoy-Navarro ${ }^{16}$, M. Titov, G.B. Yu

Laboratoire Leprince-Ringuet, CNRS/IN2P3, Ecole Polytechnique, Institut Polytechnique de Paris, Palaiseau, France

S. Ahuja, F. Beaudette, M. Bonanomi, A. Buchot Perraguin, P. Busson, A. Cappati, C. Charlot, O. Davignon, B. Diab, G. Falmagne, S. Ghosh, R. Granier de Cassagnac, 
A. Hakimi, I. Kucher, J. Motta, M. Nguyen, C. Ochando, P. Paganini, J. Rembser, R. Salerno, U. Sarkar, J.B. Sauvan, Y. Sirois, A. Tarabini, A. Zabi, A. Zghiche

Université de Strasbourg, CNRS, IPHC UMR 7178, Strasbourg, France J.-L. Agram ${ }^{17}$, J. Andrea, D. Apparu, D. Bloch, G. Bourgatte, J.-M. Brom, E.C. Chabert, C. Collard, D. Darej, J.-C. Fontaine ${ }^{17}$, U. Goerlach, C. Grimault, A.-C. Le Bihan, E. Nibigira, P. Van Hove

Institut de Physique des 2 Infinis de Lyon (IP2I ), Villeurbanne, France E. Asilar, S. Beauceron, C. Bernet, G. Boudoul, C. Camen, A. Carle, N. Chanon, D. Contardo, P. Depasse, H. El Mamouni, J. Fay, S. Gascon, M. Gouzevitch, B. Ille, I.B. Laktineh, H. Lattaud, A. Lesauvage, M. Lethuillier, L. Mirabito, S. Perries, K. Shchablo, V. Sordini, L. Torterotot, G. Touquet, M. Vander Donckt, S. Viret

\section{Georgian Technical University, Tbilisi, Georgia}

A. Khvedelidze ${ }^{13}$, I. Lomidze, Z. Tsamalaidze ${ }^{13}$

\section{RWTH Aachen University, I. Physikalisches Institut, Aachen, Germany}

V. Botta, L. Feld, K. Klein, M. Lipinski, D. Meuser, A. Pauls, N. Röwert, J. Schulz, M. Teroerde

RWTH Aachen University, III. Physikalisches Institut A, Aachen, Germany

A. Dodonova, D. Eliseev, M. Erdmann, P. Fackeldey, B. Fischer, S. Ghosh, T. Hebbeker, K. Hoepfner, F. Ivone, L. Mastrolorenzo, M. Merschmeyer, A. Meyer, G. Mocellin, S. Mondal, S. Mukherjee, D. Noll, A. Novak, T. Pook, A. Pozdnyakov, Y. Rath, H. Reithler, J. Roemer, A. Schmidt, S.C. Schuler, A. Sharma, L. Vigilante, S. Wiedenbeck, S. Zaleski

RWTH Aachen University, III. Physikalisches Institut B, Aachen, Germany

C. Dziwok, G. Flügge, W. Haj Ahmad ${ }^{18}$, O. Hlushchenko, T. Kress, A. Nowack, C. Pistone, O. Pooth, D. Roy, H. Sert, A. Stahl ${ }^{19}$, T. Ziemons, A. Zotz

\section{Deutsches Elektronen-Synchrotron, Hamburg, Germany}

H. Aarup Petersen, M. Aldaya Martin, P. Asmuss, S. Baxter, M. Bayatmakou, O. Behnke, A. Bermúdez Martínez, S. Bhattacharya, A.A. Bin Anuar, K. Borras ${ }^{20}$, D. Brunner, A. Campbell, A. Cardini, C. Cheng, F. Colombina, S. Consuegra Rodríguez, G. Correia Silva, V. Danilov, M. De Silva, L. Didukh, G. Eckerlin, D. Eckstein, L.I. Estevez Banos, O. Filatov, E. Gallo ${ }^{21}$, A. Geiser, A. Giraldi, A. Grohsjean, M. Guthoff, A. Jafari ${ }^{22}$, N.Z. Jomhari, H. Jung, A. Kasem ${ }^{20}$, M. Kasemann, H. Kaveh, C. Kleinwort, D. Krücker, W. Lange, J. Lidrych, K. Lipka, W. Lohmann ${ }^{23}$, R. Mankel, I.-A. Melzer-Pellmann, M. Mendizabal Morentin, J. Metwally, A.B. Meyer, M. Meyer, J. Mnich, A. Mussgiller, Y. Otarid, D. Pérez Adán, D. Pitzl, A. Raspereza, B. Ribeiro Lopes, J. Rübenach, A. Saggio, A. Saibel, M. Savitskyi, M. Scham ${ }^{24}$, V. Scheurer, P. Schütze, C. Schwanenberger ${ }^{21}$, M. Shchedrolosiev, R.E. Sosa Ricardo, D. Stafford, N. Tonon, M. Van De Klundert, R. Walsh, D. Walter, Y. Wen, K. Wichmann, L. Wiens, C. Wissing, S. Wuchterl

\section{University of Hamburg, Hamburg, Germany}

R. Aggleton, S. Albrecht, S. Bein, L. Benato, P. Connor, K. De Leo, M. Eich, F. Feindt, A. Fröhlich, C. Garbers, E. Garutti, P. Gunnellini, M. Hajheidari, J. Haller, A. Hinzmann, 
G. Kasieczka, R. Klanner, R. Kogler, T. Kramer, V. Kutzner, J. Lange, T. Lange, A. Lobanov, A. Malara, A. Nigamova, K.J. Pena Rodriguez, O. Rieger, P. Schleper, M. Schröder, J. Schwandt, J. Sonneveld, H. Stadie, G. Steinbrück, A. Tews, I. Zoi

Karlsruher Institut fuer Technologie, Karlsruhe, Germany

J. Bechtel, S. Brommer, E. Butz, R. Caspart, T. Chwalek, W. De Boer ${ }^{\dagger}$, A. Dierlamm, A. Droll, K. El Morabit, N. Faltermann, M. Giffels, J.o. Gosewisch, A. Gottmann, F. Hartmann ${ }^{19}$, C. Heidecker, U. Husemann, P. Keicher, R. Koppenhöfer, S. Maier, M. Metzler, S. Mitra, Th. Müller, M. Neukum, A. Nürnberg, G. Quast, K. Rabbertz, J. Rauser, D. Savoiu, M. Schnepf, D. Seith, I. Shvetsov, H.J. Simonis, R. Ulrich, J. Van Der Linden, R.F. Von Cube, M. Wassmer, M. Weber, S. Wieland, R. Wolf, S. Wozniewski, S. Wunsch

Institute of Nuclear and Particle Physics (INPP), NCSR Demokritos, Aghia Paraskevi, Greece

G. Anagnostou, G. Daskalakis, T. Geralis, A. Kyriakis, D. Loukas, A. Stakia

National and Kapodistrian University of Athens, Athens, Greece

M. Diamantopoulou, D. Karasavvas, G. Karathanasis, P. Kontaxakis, C.K. Koraka, A. Manousakis-Katsikakis, A. Panagiotou, I. Papavergou, N. Saoulidou, K. Theofilatos, E. Tziaferi, K. Vellidis, E. Vourliotis

National Technical University of Athens, Athens, Greece

G. Bakas, K. Kousouris, I. Papakrivopoulos, G. Tsipolitis, A. Zacharopoulou

University of Ioánnina, Ioánnina, Greece

K. Adamidis, I. Bestintzanos, I. Evangelou, C. Foudas, P. Gianneios, P. Katsoulis, P. Kokkas, N. Manthos, I. Papadopoulos, J. Strologas

MTA-ELTE Lendület CMS Particle and Nuclear Physics Group, Eötvös Loránd University, Budapest, Hungary

M. Csanad, K. Farkas, M.M.A. Gadallah ${ }^{25}$, S. Lökös ${ }^{26}$, P. Major, K. Mandal, A. Mehta, G. Pasztor, A.J. Rádl, O. Surányi, G.I. Veres

Wigner Research Centre for Physics, Budapest, Hungary

M. Bartók ${ }^{27}$, G. Bencze, C. Hajdu, D. Horvath ${ }^{28}$, F. Sikler, V. Veszpremi

Institute of Nuclear Research ATOMKI, Debrecen, Hungary

S. Czellar, J. Karancsi ${ }^{27}$, J. Molnar, Z. Szillasi, D. Teyssier

Institute of Physics, University of Debrecen, Debrecen, Hungary

P. Raics, Z.L. Trocsanyi ${ }^{29}$, B. Ujvari

Karoly Robert Campus, MATE Institute of Technology

T. Csorgo ${ }^{30}$, F. Nemes ${ }^{30}$, T. Novak

Indian Institute of Science (IISc), Bangalore, India

S. Choudhury, J.R. Komaragiri, D. Kumar, L. Panwar, P.C. Tiwari 
National Institute of Science Education and Research, HBNI, Bhubaneswar, India

S. Bahinipati ${ }^{31}$, C. Kar, P. Mal, T. Mishra, V.K. Muraleedharan Nair Bindhu ${ }^{32}$, A. Nayak ${ }^{32}$, P. Saha, N. Sur, S.K. Swain, D. Vats ${ }^{32}$

Panjab University, Chandigarh, India

S. Bansal, S.B. Beri, V. Bhatnagar, G. Chaudhary, S. Chauhan, N. Dhingra ${ }^{33}$, R. Gupta, A. Kaur, M. Kaur, S. Kaur, P. Kumari, M. Meena, K. Sandeep, J.B. Singh, A.K. Virdi

University of Delhi, Delhi, India

A. Ahmed, A. Bhardwaj, B.C. Choudhary, M. Gola, S. Keshri, A. Kumar, M. Naimuddin, P. Priyanka, K. Ranjan, A. Shah

Saha Institute of Nuclear Physics, HBNI, Kolkata, India

M. Bharti ${ }^{34}$, R. Bhattacharya, S. Bhattacharya, D. Bhowmik, S. Dutta, S. Dutta, B. Gomber ${ }^{35}$, M. Maity ${ }^{36}$, P. Palit, P.K. Rout, G. Saha, B. Sahu, S. Sarkar, M. Sharan, B. Singh ${ }^{34}$, S. Thakur ${ }^{34}$

Indian Institute of Technology Madras, Madras, India

P.K. Behera, S.C. Behera, P. Kalbhor, A. Muhammad, R. Pradhan, P.R. Pujahari, A. Sharma, A.K. Sikdar

Bhabha Atomic Research Centre, Mumbai, India

D. Dutta, V. Jha, V. Kumar, D.K. Mishra, K. Naskar ${ }^{37}$, P.K. Netrakanti, L.M. Pant, P. Shukla

Tata Institute of Fundamental Research-A, Mumbai, India

T. Aziz, S. Dugad, M. Kumar

Tata Institute of Fundamental Research-B, Mumbai, India

S. Banerjee, R. Chudasama, M. Guchait, S. Karmakar, S. Kumar, G. Majumder, K. Mazumdar, S. Mukherjee

Indian Institute of Science Education and Research (IISER), Pune, India K. Alpana, S. Dube, B. Kansal, A. Laha, S. Pandey, A. Rane, A. Rastogi, S. Sharma Department of Physics, Isfahan University of Technology, Isfahan, Iran H. Bakhshiansohi ${ }^{38}$, E. Khazaie, M. Zeinali ${ }^{39}$

Institute for Research in Fundamental Sciences (IPM), Tehran, Iran

S. Chenarani ${ }^{40}$, S.M. Etesami, M. Khakzad, M. Mohammadi Najafabadi

University College Dublin, Dublin, Ireland

M. Grunewald

INFN Sezione di Bari ${ }^{a}$, Università di Bari ${ }^{b}$, Politecnico di Bari ${ }^{c}$, Bari, Italy

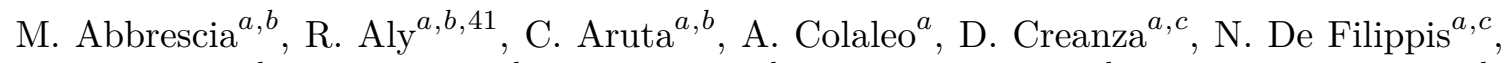
M. De Palma ${ }^{a, b}$, A. Di Florio ${ }^{a, b}$, A. Di Pilato ${ }^{a, b}$, W. Elmetenawee ${ }^{a, b}$, L. Fiore $^{a}$, A. Gelmi $^{a, b}$,

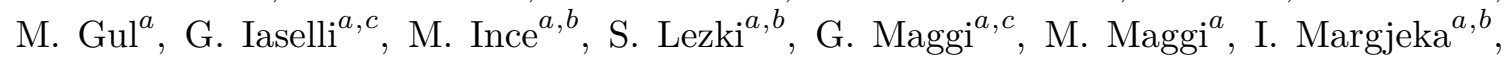




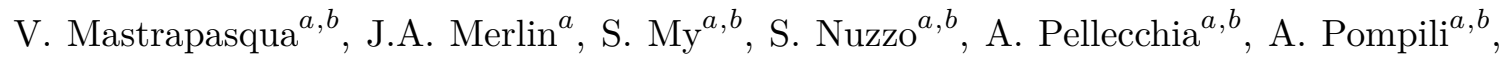
G. Pugliese ${ }^{a, c}$, D. Ramos, A. Ranieri ${ }^{a}$, G. Selvaggi ${ }^{a, b}$, L. Silvestris $^{a}$, F.M. Simone ${ }^{a, b}$, R. Venditti ${ }^{a}$, P. Verwilligen ${ }^{a}$

\section{INFN Sezione di Bologna ${ }^{a}$, Università di Bologna ${ }^{b}$, Bologna, Italy}

G. Abbiendi $^{a}$, C. Battilana ${ }^{a, b}$, D. Bonacorsi ${ }^{a, b}$, L. Borgonovi ${ }^{a}$, L. Brigliadori ${ }^{a}$, R. Campanini ${ }^{a, b}$, P. Capiluppi ${ }^{a, b}$, A. Castro ${ }^{a, b}$, F.R. Cavallo ${ }^{a}$, M. Cuffiani ${ }^{a, b}$, G.M. Dallavalle ${ }^{a}$, T. Diotalevi ${ }^{a, b}$, F. Fabbri ${ }^{a}$, A. Fanfani ${ }^{a, b}$, P. Giacomelli $^{a}$, L. Giommi $^{a, b}{ }^{,}$ C. Grandi ${ }^{a}$, L. Guiducci ${ }^{a, b}$, S. Lo Meo ${ }^{a, 42}$, L. Lunerti ${ }^{a, b}$, S. Marcellini ${ }^{a}$, G. Masetti ${ }^{a}$,

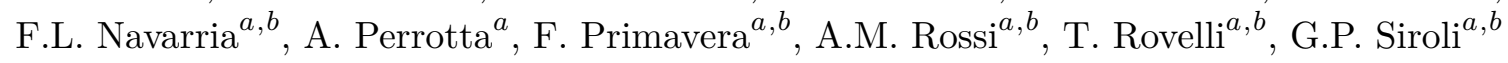

INFN Sezione di Catania ${ }^{a}$, Università di Catania ${ }^{b}$, Catania, Italy

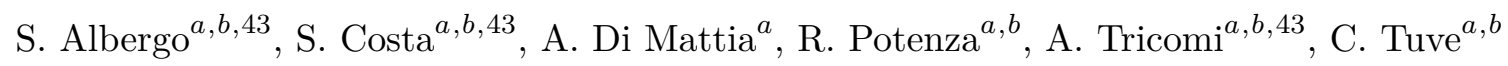
INFN Sezione di Firenze ${ }^{a}$, Università di Firenze ${ }^{b}$, Firenze, Italy

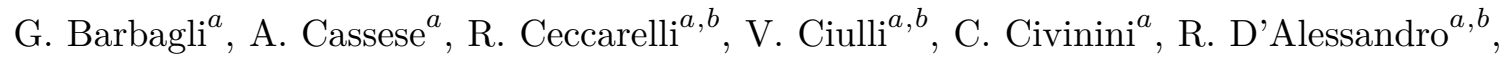
E. Focardi ${ }^{a, b}$, G. Latino ${ }^{a, b}$, P. Lenzi ${ }^{a, b}$, M. Lizzo ${ }^{a, b}$, M. Meschini ${ }^{a}$, S. Paoletti ${ }^{a}$, R. Seidita ${ }^{a, b}$, G. Sguazzoni ${ }^{a}$, L. Viliani $^{a}$

INFN Laboratori Nazionali di Frascati, Frascati, Italy

L. Benussi, S. Bianco, D. Piccolo

INFN Sezione di Genova ${ }^{a}$, Università di Genova ${ }^{b}$, Genova, Italy

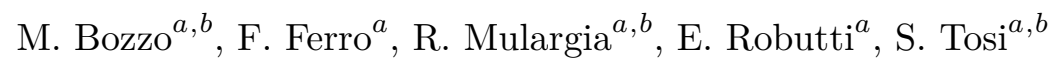

INFN Sezione di Milano-Bicocca $^{a}$, Università di Milano-Bicocca ${ }^{b}$, Milano, Italy

A. Benaglia $^{a}$, G. Boldrini, F. Brivio ${ }^{a, b}$, F. Cetorelli ${ }^{a, b}$, F. De Guio ${ }^{a, b}$, M.E. Dinardo $^{a, b}$, P. Dini ${ }^{a}$, S. Gennai ${ }^{a}$, A. Ghezzi ${ }^{a, b}$, P. Govoni ${ }^{a, b}$, L. Guzzi ${ }^{a, b}$, M.T. Lucchini ${ }^{a, b}$, M. Malberti ${ }^{a}$, S. Malvezzi ${ }^{a}$, A. Massironi ${ }^{a}$, D. Menasce ${ }^{a}$, L. Moroni $^{a}$, M. Paganoni $^{a}{ }^{a}$, D. Pedrini ${ }^{a}$, B.S. Pinolini, S. Ragazzi ${ }^{a, b}$, N. Redaelli ${ }^{a}$, T. Tabarelli de Fatis ${ }^{a, b}$, D. Valsecchi ${ }^{a, b, 19}$, D. Zuolo ${ }^{a, b}$

INFN Sezione di Napoli ${ }^{a}$, Università di Napoli 'Federico II' ${ }^{b}$, Napoli, Italy, Università della Basilicata ${ }^{c}$, Potenza, Italy, Università G. Marconi ${ }^{d}$, Roma, Italy

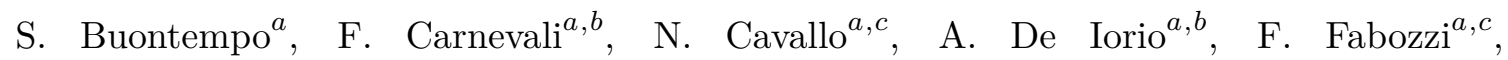

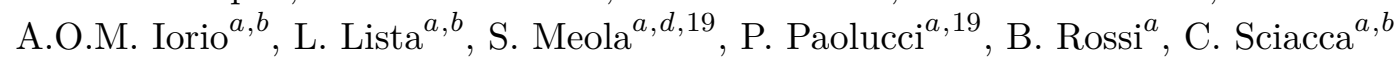

INFN Sezione di Padova ${ }^{a}$, Università di Padova ${ }^{b}$, Padova, Italy, Università di Trento ${ }^{c}$, Trento, Italy

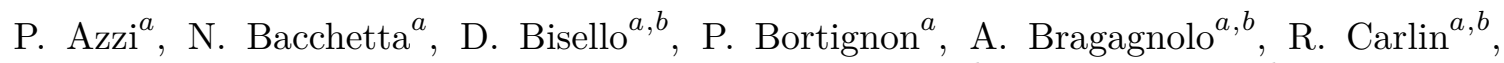
P. Checchia ${ }^{a}$, T. Dorigo ${ }^{a}$, U. Dosselli ${ }^{a}$, F. Gasparini ${ }^{a, b}$, U. Gasparini ${ }^{a, b}$, G. Grosso, S.Y. Hoh ${ }^{a, b}$, L. Layer ${ }^{a, 44}$, E. Lusiani, M. Margoni ${ }^{a, b}$, A.T. Meneguzzo ${ }^{a, b}$, J. Pazzini $^{a, b}$,

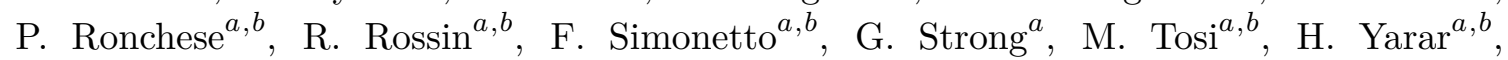
M. Zanetti ${ }^{a, b}$, P. Zotto ${ }^{a, b}$, A. Zucchetta ${ }^{a, b}$, G. Zumerle ${ }^{a, b}$ 
INFN Sezione di Pavia ${ }^{a}$, Università di Pavia ${ }^{b}$, Pavia, Italy

C. Aime ${ }^{a, b}$, A. Braghieri ${ }^{a}$, S. Calzaferri ${ }^{a, b}$, D. Fiorina ${ }^{a, b}$, P. Montagna ${ }^{a, b}$, S.P. Ratti ${ }^{a, b}$,

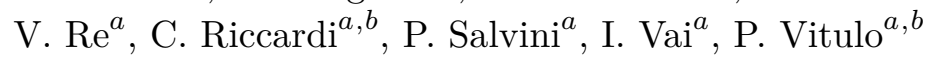

INFN Sezione di Perugia ${ }^{a}$, Università di Perugia ${ }^{b}$, Perugia, Italy

P. Asenov ${ }^{a, 45}$, G.M. Bilei ${ }^{a}$, D. Ciangottini ${ }^{a, b}$, L. Fanò ${ }^{a, b}$, P. Lariccia $^{a, b}$, M. Magherini $^{b}$, G. Mantovani ${ }^{a, b}$, V. Mariani ${ }^{a, b}$, M. Menichelli ${ }^{a}$, F. Moscatelli ${ }^{a, 45}$, A. Piccinelli ${ }^{a, b}$,

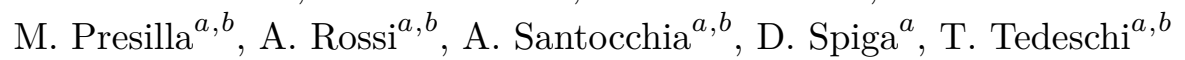

INFN Sezione di Pisa ${ }^{a}$, Università di Pisa ${ }^{b}$, Scuola Normale Superiore di Pisa ${ }^{c}$, Pisa Italy, Università di Siena ${ }^{d}$, Siena, Italy

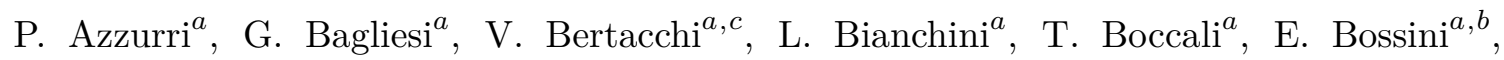
R. Castaldi $^{a}$, M.A. Ciocci ${ }^{a, b}$, V. D'Amante ${ }^{a, d}$, R. Dell'Orso ${ }^{a}$, M.R. Di Domenico ${ }^{a, d}$,

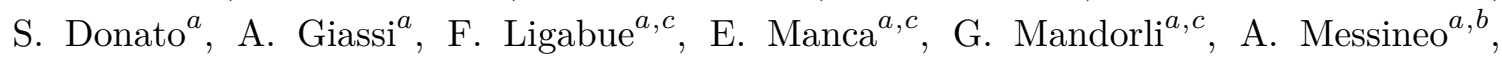
F. Palla ${ }^{a}$, S. Parolia ${ }^{a, b}$, G. Ramirez-Sanchez ${ }^{a, c}$, A. Rizzi ${ }^{a, b}$, G. Rolandi ${ }^{a, c}$, S. Roy Chowdhury ${ }^{a, c}$, A. Scribano $^{a}$, N. Shafiei ${ }^{a, b}$, P. Spagnolo $^{a}$, R. Tenchini $^{a}$, G. Tonelli $^{a, b}$, N. Turini ${ }^{a, d}$, A. Venturi $^{a}$, P.G. Verdini ${ }^{a}$

INFN Sezione di Roma ${ }^{a}$, Sapienza Università di Roma ${ }^{b}$, Rome, Italy

P. Barria ${ }^{a}$, M. Campana ${ }^{a, b}$, F. Cavallari ${ }^{a}$, D. Del Re ${ }^{a, b}$, E. Di Marco ${ }^{a}$, M. Diemoz $^{a}$, E. Longo $^{a, b}$, P. Meridiani $^{a}$, G. Organtini ${ }^{a, b}$, F. Pandolfi ${ }^{a}$, R. Paramatti $^{a, b}$, C. Quaranta $^{a, b}$,

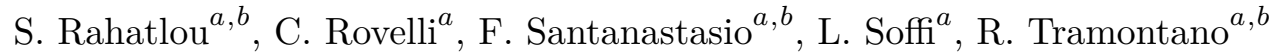

INFN Sezione di Torino ${ }^{a}$, Università di Torino ${ }^{b}$, Torino, Italy, Università del Piemonte Orientale ${ }^{c}$, Novara, Italy

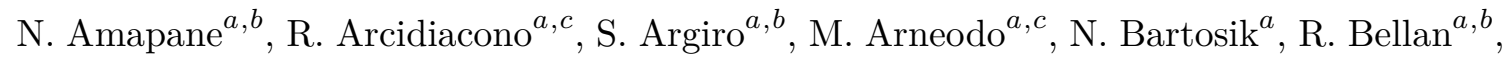

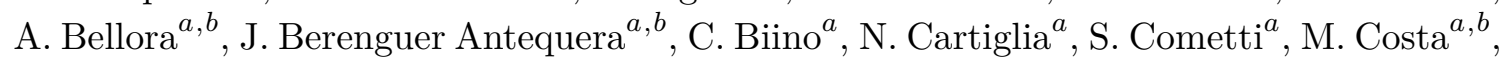
R. Covarelli ${ }^{a, b}$, N. Demaria ${ }^{a}$, B. Kiani ${ }^{a, b}$, F. Legger ${ }^{a}$, C. Mariotti $^{a}$, S. Maselli ${ }^{a}$, E. Migliore $^{a, b}$, E. Monteil ${ }^{a, b}$, M. Monteno ${ }^{a}$, M.M. Obertino ${ }^{a, b}$, G. Ortona $^{a}$, L. Pacher ${ }^{a, b}$, N. Pastrone ${ }^{a}$, M. Pelliccioni ${ }^{a}$, G.L. Pinna Angioni ${ }^{a, b}$, M. Ruspa ${ }^{a, c}$, K. Shchelina ${ }^{a}$,

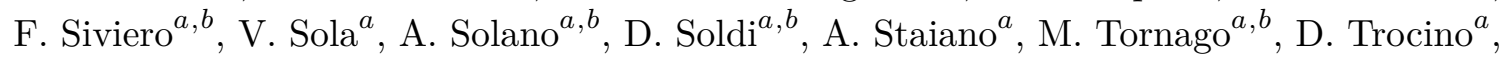
A. Vagnerini ${ }^{a, b}$

INFN Sezione di Trieste ${ }^{a}$, Università di Trieste ${ }^{b}$, Trieste, Italy

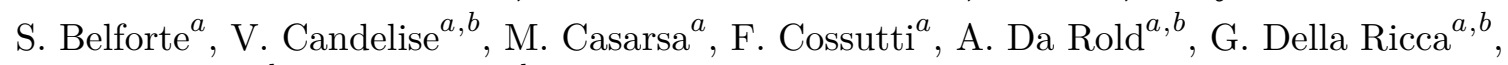
G. Sorrentino $^{a, b}$, F. Vazzoler ${ }^{a, b}$

\section{Kyungpook National University, Daegu, Korea}

S. Dogra, C. Huh, B. Kim, D.H. Kim, G.N. Kim, J. Kim, J. Lee, S.W. Lee, C.S. Moon, Y.D. Oh, S.I. Pak, B.C. Radburn-Smith, S. Sekmen, Y.C. Yang

Chonnam National University, Institute for Universe and Elementary Particles, Kwangju, Korea

H. Kim, D.H. Moon

Hanyang University, Seoul, Korea

B. Francois, T.J. Kim, J. Park 
Korea University, Seoul, Korea

S. Cho, S. Choi, Y. Go, B. Hong, K. Lee, K.S. Lee, J. Lim, J. Park, S.K. Park, J. Yoo

Kyung Hee University, Department of Physics, Seoul, Republic of Korea

J. Goh, A. Gurtu

Sejong University, Seoul, Korea

H.S. Kim, Y. Kim

Seoul National University, Seoul, Korea

J. Almond, J.H. Bhyun, J. Choi, S. Jeon, J. Kim, J.S. Kim, S. Ko, H. Kwon, H. Lee, S. Lee, B.H. Oh, M. Oh, S.B. Oh, H. Seo, U.K. Yang, I. Yoon

University of Seoul, Seoul, Korea

W. Jang, D.Y. Kang, Y. Kang, S. Kim, B. Ko, J.S.H. Lee, Y. Lee, I.C. Park, Y. Roh, M.S. Ryu, D. Song, I.J. Watson, S. Yang

Yonsei University, Department of Physics, Seoul, Korea

S. Ha, H.D. Yoo

Sungkyunkwan University, Suwon, Korea

M. Choi, H. Lee, Y. Lee, I. Yu

College of Engineering and Technology, American University of the Middle East (AUM), Egaila, Kuwait

T. Beyrouthy, Y. Maghrbi

Riga Technical University, Riga, Latvia

T. Torims, V. Veckalns ${ }^{46}$

Vilnius University, Vilnius, Lithuania

M. Ambrozas, A. Carvalho Antunes De Oliveira, A. Juodagalvis, A. Rinkevicius, G. Tamulaitis

National Centre for Particle Physics, Universiti Malaya, Kuala Lumpur, Malaysia

N. Bin Norjoharuddeen, W.A.T. Wan Abdullah, M.N. Yusli, Z. Zolkapli

Universidad de Sonora (UNISON), Hermosillo, Mexico

J.F. Benitez, A. Castaneda Hernandez, M. León Coello, J.A. Murillo Quijada, A. Sehrawat, L. Valencia Palomo

Centro de Investigacion y de Estudios Avanzados del IPN, Mexico City, Mexico G. Ayala, H. Castilla-Valdez, E. De La Cruz-Burelo, I. Heredia-De La Cruz ${ }^{47}$, R. LopezFernandez, C.A. Mondragon Herrera, D.A. Perez Navarro, A. Sanchez-Hernandez

Universidad Iberoamericana, Mexico City, Mexico

S. Carrillo Moreno, C. Oropeza Barrera, F. Vazquez Valencia

Benemerita Universidad Autonoma de Puebla, Puebla, Mexico

I. Pedraza, H.A. Salazar Ibarguen, C. Uribe Estrada 
University of Montenegro, Podgorica, Montenegro

J. Mijuskovic ${ }^{48}$, N. Raicevic

University of Auckland, Auckland, New Zealand

D. Krofcheck

University of Canterbury, Christchurch, New Zealand

P.H. Butler

National Centre for Physics, Quaid-I-Azam University, Islamabad, Pakistan

A. Ahmad, M.I. Asghar, A. Awais, M.I.M. Awan, H.R. Hoorani, W.A. Khan, M.A. Shah, M. Shoaib, M. Waqas

AGH University of Science and Technology Faculty of Computer Science, Electronics and Telecommunications, Krakow, Poland

V. Avati, L. Grzanka, M. Malawski

National Centre for Nuclear Research, Swierk, Poland

H. Bialkowska, M. Bluj, B. Boimska, M. Górski, M. Kazana, M. Szleper, P. Zalewski

Institute of Experimental Physics, Faculty of Physics, University of Warsaw, Warsaw, Poland

K. Bunkowski, K. Doroba, A. Kalinowski, M. Konecki, J. Krolikowski

Laboratório de Instrumentação e Física Experimental de Partículas, Lisboa, Portugal

M. Araujo, P. Bargassa, D. Bastos, A. Boletti, P. Faccioli, M. Gallinaro, J. Hollar, N. Leonardo, T. Niknejad, M. Pisano, J. Seixas, O. Toldaiev, J. Varela

Joint Institute for Nuclear Research, Dubna, Russia

S. Afanasiev, D. Budkouski, I. Golutvin, I. Gorbunov, V. Karjavine, V. Korenkov, A. Lanev, A. Malakhov, V. Matveev ${ }^{49,50}$, V. Palichik, V. Perelygin, M. Savina, D. Seitova, V. Shalaev, S. Shmatov, S. Shulha, V. Smirnov, O. Teryaev, N. Voytishin, B.S. Yuldashev ${ }^{51}$,

A. Zarubin, I. Zhizhin

Petersburg Nuclear Physics Institute, Gatchina (St. Petersburg), Russia

G. Gavrilov, V. Golovtcov, Y. Ivanov, V. Kim ${ }^{52}$, E. Kuznetsova ${ }^{53}$, V. Murzin, V. Oreshkin, I. Smirnov, D. Sosnov, V. Sulimov, L. Uvarov, S. Volkov, A. Vorobyev

Institute for Nuclear Research, Moscow, Russia

Yu. Andreev, A. Dermenev, S. Gninenko, N. Golubev, A. Karneyeu, D. Kirpichnikov, M. Kirsanov, N. Krasnikov, A. Pashenkov, G. Pivovarov, A. Toropin

Institute for Theoretical and Experimental Physics named by A.I. Alikhanov of NRC 'Kurchatov Institute', Moscow, Russia

V. Epshteyn, V. Gavrilov, N. Lychkovskaya, A. Nikitenko ${ }^{54}$, V. Popov, A. Stepennov, M. Toms, E. Vlasov, A. Zhokin

Moscow Institute of Physics and Technology, Moscow, Russia

T. Aushev 
National Research Nuclear University 'Moscow Engineering Physics Institute' (MEPhI), Moscow, Russia

O. Bychkova, R. Chistov ${ }^{55}$, M. Danilov ${ }^{56}$, A. Oskin, S. Polikarpov ${ }^{56}$, D. Selivanova

P.N. Lebedev Physical Institute, Moscow, Russia

V. Andreev, M. Azarkin, I. Dremin, M. Kirakosyan, A. Terkulov

Skobeltsyn Institute of Nuclear Physics, Lomonosov Moscow State University, Moscow, Russia

A. Belyaev, E. Boos, V. Bunichev, M. Dubinin ${ }^{57}$, L. Dudko, A. Gribushin, V. Klyukhin, O. Kodolova, I. Lokhtin, S. Obraztsov, M. Perfilov, V. Savrin, A. Snigirev

Novosibirsk State University (NSU), Novosibirsk, Russia

V. Blinov ${ }^{58}$, T. Dimova ${ }^{58}$, L. Kardapoltsev ${ }^{58}$, A. Kozyrev ${ }^{58}$, I. Ovtin ${ }^{58}$, Y. Skovpen ${ }^{58}$

Institute for High Energy Physics of National Research Centre 'Kurchatov Institute', Protvino, Russia

I. Azhgirey, I. Bayshev, D. Elumakhov, V. Kachanov, D. Konstantinov, P. Mandrik, V. Petrov, R. Ryutin, S. Slabospitskii, A. Sobol, S. Troshin, N. Tyurin, A. Uzunian, A. Volkov

National Research Tomsk Polytechnic University, Tomsk, Russia

A. Babaev, V. Okhotnikov

Tomsk State University, Tomsk, Russia

V. Borshch, V. Ivanchenko, E. Tcherniaev

University of Belgrade: Faculty of Physics and VINCA Institute of Nuclear Sciences, Belgrade, Serbia

P. Adzic ${ }^{59}$, M. Dordevic, P. Milenovic, J. Milosevic

Centro de Investigaciones Energéticas Medioambientales y Tecnológicas (CIEMAT), Madrid, Spain

M. Aguilar-Benitez, J. Alcaraz Maestre, A. Álvarez Fernández, I. Bachiller, M. Barrio Luna, Cristina F. Bedoya, C.A. Carrillo Montoya, M. Cepeda, M. Cerrada, N. Colino, B. De La Cruz, A. Delgado Peris, J.P. Fernández Ramos, J. Flix, M.C. Fouz, O. Gonzalez Lopez, S. Goy Lopez, J.M. Hernandez, M.I. Josa, J. León Holgado, D. Moran, Á. Navarro Tobar, C. Perez Dengra, A. Pérez-Calero Yzquierdo, J. Puerta Pelayo, I. Redondo, L. Romero, S. Sánchez Navas, L. Urda Gómez, C. Willmott

Universidad Autónoma de Madrid, Madrid, Spain

J.F. de Trocóniz, R. Reyes-Almanza

Universidad de Oviedo, Instituto Universitario de Ciencias y Tecnologías Espaciales de Asturias (ICTEA), Oviedo, Spain

B. Alvarez Gonzalez, J. Cuevas, C. Erice, J. Fernandez Menendez, S. Folgueras, I. Gonzalez Caballero, J.R. González Fernández, E. Palencia Cortezon, C. Ramón Álvarez, V. Rodríguez Bouza, A. Soto Rodríguez, A. Trapote, N. Trevisani, C. Vico Villalba 
Instituto de Física de Cantabria (IFCA), CSIC-Universidad de Cantabria, Santander, Spain

J.A. Brochero Cifuentes, I.J. Cabrillo, A. Calderon, J. Duarte Campderros, M. Fernandez, C. Fernandez Madrazo, P.J. Fernández Manteca, A. García Alonso, G. Gomez, C. Martinez Rivero, P. Martinez Ruiz del Arbol, F. Matorras, Pablo Matorras-Cuevas,

J. Piedra Gomez, C. Prieels, T. Rodrigo, A. Ruiz-Jimeno, L. Scodellaro, I. Vila, J.M. Vizan Garcia

\section{University of Colombo, Colombo, Sri Lanka}

M.K. Jayananda, B. Kailasapathy ${ }^{60}$, D.U.J. Sonnadara, D.D.C. Wickramarathna

\section{University of Ruhuna, Department of Physics, Matara, Sri Lanka}

W.G.D. Dharmaratna, K. Liyanage, N. Perera, N. Wickramage

\section{CERN, European Organization for Nuclear Research, Geneva, Switzerland}

T.K. Aarrestad, D. Abbaneo, J. Alimena, E. Auffray, G. Auzinger, J. Baechler, P. Baillon ${ }^{\dagger}$, D. Barney, J. Bendavid, M. Bianco, A. Bocci, T. Camporesi, M. Capeans Garrido, G. Cerminara, N. Chernyavskaya, S.S. Chhibra, M. Cipriani, L. Cristella, D. d'Enterria, A. Dabrowski, A. David, A. De Roeck, M.M. Defranchis, M. Deile, M. Dobson, M. Dünser, N. Dupont, A. Elliott-Peisert, N. Emriskova, F. Fallavollita ${ }^{61}$, D. Fasanella, A. Florent, G. Franzoni, W. Funk, S. Giani, D. Gigi, K. Gill, F. Glege, L. Gouskos, M. Haranko, J. Hegeman, V. Innocente, T. James, P. Janot, J. Kaspar, J. Kieseler, M. Komm, N. Kratochwil, C. Lange, S. Laurila, P. Lecoq, A. Lintuluoto, K. Long, C. Lourenço, B. Maier, L. Malgeri, S. Mallios, M. Mannelli, A.C. Marini, F. Meijers, S. Mersi, E. Meschi, F. Moortgat, M. Mulders, S. Orfanelli, L. Orsini, F. Pantaleo, L. Pape, E. Perez, M. Peruzzi, A. Petrilli, G. Petrucciani, A. Pfeiffer, M. Pierini, D. Piparo, M. Pitt, H. Qu, T. Quast, D. Rabady, A. Racz, G. Reales Gutiérrez, M. Rieger, M. Rovere, H. Sakulin, J. SalfeldNebgen, S. Scarfi, C. Schäfer, C. Schwick, M. Selvaggi, A. Sharma, P. Silva, W. Snoeys, P. Sphicas ${ }^{62}$, S. Summers, K. Tatar, V.R. Tavolaro, D. Treille, P. Tropea, A. Tsirou, G.P. Van Onsem, J. Wanczyk ${ }^{63}$, K.A. Wozniak, W.D. Zeuner

\section{Paul Scherrer Institut, Villigen, Switzerland}

L. Caminada ${ }^{64}$, A. Ebrahimi, W. Erdmann, R. Horisberger, Q. Ingram, H.C. Kaestli, D. Kotlinski, U. Langenegger, M. Missiroli ${ }^{64}$, L. Noehte ${ }^{64}$, T. Rohe

ETH Zurich - Institute for Particle Physics and Astrophysics (IPA), Zurich, Switzerland

K. Androsov ${ }^{63}$, M. Backhaus, P. Berger, A. Calandri, A. De Cosa, G. Dissertori, M. Dittmar, M. Donegà, C. Dorfer, F. Eble, K. Gedia, F. Glessgen, T.A. Gómez Espinosa, C. Grab, D. Hits, W. Lustermann, A.-M. Lyon, R.A. Manzoni, L. Marchese, C. Martin Perez, M.T. Meinhard, F. Nessi-Tedaldi, J. Niedziela, F. Pauss, V. Perovic, S. Pigazzini, M.G. Ratti, M. Reichmann, C. Reissel, T. Reitenspiess, B. Ristic, D. Ruini, D.A. Sanz Becerra, V. Stampf, J. Steggemann ${ }^{63}$, R. Wallny, D.H. Zhu 
Universität Zürich, Zurich, Switzerland

C. Amsler ${ }^{65}$, P. Bärtschi, C. Botta, D. Brzhechko, M.F. Canelli, K. Cormier, A. De Wit,

R. Del Burgo, J.K. Heikkilä, M. Huwiler, W. Jin, A. Jofrehei, B. Kilminster, S. Leontsinis, S.P. Liechti, A. Macchiolo, P. Meiring, V.M. Mikuni, U. Molinatti, I. Neutelings, A. Reimers, P. Robmann, S. Sanchez Cruz, K. Schweiger, Y. Takahashi

\section{National Central University, Chung-Li, Taiwan}

C. Adloff ${ }^{66}$, C.M. Kuo, W. Lin, A. Roy, T. Sarkar ${ }^{36}$, S.S. Yu

National Taiwan University (NTU), Taipei, Taiwan

L. Ceard, Y. Chao, K.F. Chen, P.H. Chen, W.-S. Hou, Y.y. Li, R.-S. Lu, E. Paganis,

A. Psallidas, A. Steen, H.y. Wu, E. Yazgan, P.r. Yu

Chulalongkorn University, Faculty of Science, Department of Physics, Bangkok, Thailand

B. Asavapibhop, C. Asawatangtrakuldee, N. Srimanobhas

Çukurova University, Physics Department, Science and Art Faculty, Adana, Turkey

F. Boran, S. Damarseckin ${ }^{67}$, Z.S. Demiroglu, F. Dolek, I. Dumanoglu ${ }^{68}$, E. Eskut, Y. Guler ${ }^{69}$, E. Gurpinar Guler ${ }^{69}$, C. Isik, O. Kara, A. Kayis Topaksu, U. Kiminsu, G. Onengut, K. Ozdemir ${ }^{70}$, A. Polatoz, A.E. Simsek, B. Tali ${ }^{71}$, U.G. Tok, S. Turkcapar, I.S. Zorbakir, C. Zorbilmez

Middle East Technical University, Physics Department, Ankara, Turkey

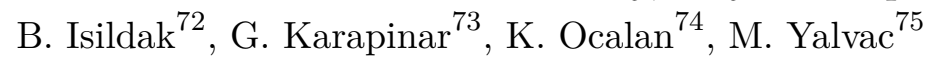

Bogazici University, Istanbul, Turkey

B. Akgun, I.O. Atakisi, E. Gülmez, M. Kaya ${ }^{76}$, O. Kaya ${ }^{77}$, Ö. Özçelik, S. Tekten ${ }^{78}$, E.A. Yetkin ${ }^{79}$

Istanbul Technical University, Istanbul, Turkey

A. Cakir, K. Cankocak ${ }^{68}$, Y. Komurcu, S. Sen ${ }^{80}$

Istanbul University, Istanbul, Turkey

S. Cerci ${ }^{71}$, I. $\operatorname{Hos}^{81}$, B. Kaynak, S. Ozkorucuklu, D. Sunar Cerci ${ }^{71}$

Institute for Scintillation Materials of National Academy of Science of Ukraine, Kharkov, Ukraine

B. Grynyov

National Scientific Center, Kharkov Institute of Physics and Technology, Kharkov, Ukraine

L. Levchuk

University of Bristol, Bristol, United Kingdom

D. Anthony, E. Bhal, S. Bologna, J.J. Brooke, A. Bundock, E. Clement, D. Cussans,

H. Flacher, J. Goldstein, G.P. Heath, H.F. Heath, L. Kreczko, B. Krikler, S. Paramesvaran, S. Seif El Nasr-Storey, V.J. Smith, N. Stylianou ${ }^{82}$, K. Walkingshaw Pass, R. White 


\section{Rutherford Appleton Laboratory, Didcot, United Kingdom}

K.W. Bell, A. Belyaev ${ }^{83}$, C. Brew, R.M. Brown, D.J.A. Cockerill, C. Cooke, K.V. Ellis, K. Harder, S. Harper, M.l. Holmberg ${ }^{84}$, J. Linacre, K. Manolopoulos, D.M. Newbold, E. Olaiya, D. Petyt, T. Reis, T. Schuh, C.H. Shepherd-Themistocleous, I.R. Tomalin, T. Williams

\section{Imperial College, London, United Kingdom}

R. Bainbridge, P. Bloch, S. Bonomally, J. Borg, S. Breeze, O. Buchmuller, V. Cepaitis, G.S. Chahal ${ }^{85}$, D. Colling, P. Dauncey, G. Davies, M. Della Negra, S. Fayer, G. Fedi, G. Hall, M.H. Hassanshahi, G. Iles, J. Langford, L. Lyons, A.-M. Magnan, S. Malik, A. Martelli, D.G. Monk, J. Nash ${ }^{86}$, M. Pesaresi, D.M. Raymond, A. Richards, A. Rose, E. Scott, C. Seez, A. Shtipliyski, A. Tapper, K. Uchida, T. Virdee ${ }^{19}$, M. Vojinovic, N. Wardle, S.N. Webb, D. Winterbottom

\section{Brunel University, Uxbridge, United Kingdom}

K. Coldham, J.E. Cole, A. Khan, P. Kyberd, I.D. Reid, L. Teodorescu, S. Zahid

Baylor University, Waco, U.S.A.

S. Abdullin, A. Brinkerhoff, B. Caraway, J. Dittmann, K. Hatakeyama, A.R. Kanuganti, B. McMaster, N. Pastika, M. Saunders, S. Sawant, C. Sutantawibul, J. Wilson

Catholic University of America, Washington, DC, U.S.A.

R. Bartek, A. Dominguez, R. Uniyal, A.M. Vargas Hernandez

The University of Alabama, Tuscaloosa, U.S.A.

A. Buccilli, S.I. Cooper, D. Di Croce, S.V. Gleyzer, C. Henderson, C.U. Perez, P. Rumerio ${ }^{87}$, C. West

\section{Boston University, Boston, U.S.A.}

A. Akpinar, A. Albert, D. Arcaro, C. Cosby, Z. Demiragli, E. Fontanesi, D. Gastler, S. May, J. Rohlf, K. Salyer, D. Sperka, D. Spitzbart, I. Suarez, A. Tsatsos, S. Yuan, D. Zou

Brown University, Providence, U.S.A.

G. Benelli, B. Burkle, X. Coubez $^{20}$, D. Cutts, M. Hadley, U. Heintz, J.M. Hogan ${ }^{88}$, T. KWON, G. Landsberg, K.T. Lau, D. Li, M. Lukasik, J. Luo, M. Narain, N. Pervan, S. Sagir ${ }^{89}$, F. Simpson, E. Usai, W.Y. Wong, X. Yan, D. Yu, W. Zhang

University of California, Davis, Davis, U.S.A.

J. Bonilla, C. Brainerd, R. Breedon, M. Calderon De La Barca Sanchez, M. Chertok, J. Conway, P.T. Cox, R. Erbacher, G. Haza, F. Jensen, O. Kukral, R. Lander, M. Mulhearn, D. Pellett, B. Regnery, D. Taylor, Y. Yao, F. Zhang

\section{University of California, Los Angeles, U.S.A.}

M. Bachtis, R. Cousins, A. Datta, D. Hamilton, J. Hauser, M. Ignatenko, M.A. Iqbal, T. Lam, W.A. Nash, S. Regnard, D. Saltzberg, B. Stone, V. Valuev

University of California, Riverside, Riverside, U.S.A.

K. Burt, Y. Chen, R. Clare, J.W. Gary, M. Gordon, G. Hanson, G. Karapostoli, O.R. Long, N. Manganelli, M. Olmedo Negrete, W. Si, S. Wimpenny, Y. Zhang 
University of California, San Diego, La Jolla, U.S.A.

J.G. Branson, P. Chang, S. Cittolin, S. Cooperstein, N. Deelen, D. Diaz, J. Duarte, R. Gerosa, L. Giannini, D. Gilbert, J. Guiang, R. Kansal, V. Krutelyov, R. Lee, J. Letts, M. Masciovecchio, M. Pieri, B.V. Sathia Narayanan, V. Sharma, M. Tadel, A. Vartak, F. Würthwein, Y. Xiang, A. Yagil

University of California, Santa Barbara - Department of Physics, Santa Barbara, U.S.A.

N. Amin, C. Campagnari, M. Citron, A. Dorsett, V. Dutta, J. Incandela, M. Kilpatrick, J. Kim, B. Marsh, H. Mei, M. Oshiro, M. Quinnan, J. Richman, U. Sarica, F. Setti, J. Sheplock, D. Stuart, S. Wang

California Institute of Technology, Pasadena, U.S.A.

A. Bornheim, O. Cerri, I. Dutta, J.M. Lawhorn, N. Lu, J. Mao, H.B. Newman, T.Q. Nguyen, M. Spiropulu, J.R. Vlimant, C. Wang, S. Xie, Z. Zhang, R.Y. Zhu

Carnegie Mellon University, Pittsburgh, U.S.A.

J. Alison, S. An, M.B. Andrews, P. Bryant, T. Ferguson, A. Harilal, C. Liu, T. Mudholkar, M. Paulini, A. Sanchez, W. Terrill

University of Colorado Boulder, Boulder, U.S.A.

J.P. Cumalat, W.T. Ford, A. Hassani, E. MacDonald, R. Patel, A. Perloff, C. Savard, K. Stenson, K.A. Ulmer, S.R. Wagner

Cornell University, Ithaca, U.S.A.

J. Alexander, S. Bright-thonney, Y. Cheng, D.J. Cranshaw, S. Hogan, J. Monroy, J.R. Patterson, D. Quach, J. Reichert, M. Reid, A. Ryd, W. Sun, J. Thom, P. Wittich, R. Zou

Fermi National Accelerator Laboratory, Batavia, U.S.A.

M. Albrow, M. Alyari, G. Apollinari, A. Apresyan, A. Apyan, S. Banerjee, L.A.T. Bauerdick, D. Berry, J. Berryhill, P.C. Bhat, K. Burkett, J.N. Butler, A. Canepa, G.B. Cerati, H.W.K. Cheung, F. Chlebana, M. Cremonesi, K.F. Di Petrillo, V.D. Elvira, Y. Feng, J. Freeman, Z. Gecse, L. Gray, D. Green, S. Grünendahl, O. Gutsche, R.M. Harris, R. Heller, T.C. Herwig, J. Hirschauer, B. Jayatilaka, S. Jindariani, M. Johnson, U. Joshi, T. Klijnsma, B. Klima, K.H.M. Kwok, S. Lammel, D. Lincoln, R. Lipton, T. Liu, C. Madrid, K. Maeshima, C. Mantilla, D. Mason, P. McBride, P. Merkel, S. Mrenna, S. Nahn, J. Ngadiuba, V. O'Dell, V. Papadimitriou, K. Pedro, C. Pena ${ }^{57}$, O. Prokofyev, F. Ravera, A. Reinsvold Hall, L. Ristori, E. Sexton-Kennedy, N. Smith, A. Soha, W.J. Spalding, L. Spiegel, S. Stoynev, J. Strait, L. Taylor, S. Tkaczyk, N.V. Tran, L. Uplegger, E.W. Vaandering, H.A. Weber

\section{University of Florida, Gainesville, U.S.A.}

D. Acosta, P. Avery, D. Bourilkov, L. Cadamuro, V. Cherepanov, F. Errico, R.D. Field, D. Guerrero, B.M. Joshi, M. Kim, E. Koenig, J. Konigsberg, A. Korytov, K.H. Lo, K. Matchev, N. Menendez, G. Mitselmakher, A. Muthirakalayil Madhu, N. Rawal, D. Rosenzweig, S. Rosenzweig, J. Rotter, K. Shi, J. Sturdy, J. Wang, E. Yigitbasi, X. Zuo 
Florida State University, Tallahassee, U.S.A.

T. Adams, A. Askew, R. Habibullah, V. Hagopian, K.F. Johnson, R. Khurana, T. Kolberg, G. Martinez, H. Prosper, C. Schiber, O. Viazlo, R. Yohay, J. Zhang

Florida Institute of Technology, Melbourne, U.S.A.

M.M. Baarmand, S. Butalla, T. Elkafrawy ${ }^{90}$, M. Hohlmann, R. Kumar Verma, D. Noonan, M. Rahmani, F. Yumiceva

University of Illinois at Chicago (UIC), Chicago, U.S.A.

M.R. Adams, H. Becerril Gonzalez, R. Cavanaugh, X. Chen, S. Dittmer, O. Evdokimov, C.E. Gerber, D.A. Hangal, D.J. Hofman, A.H. Merrit, C. Mills, G. Oh, T. Roy, S. Rudrabhatla, M.B. Tonjes, N. Varelas, J. Viinikainen, X. Wang, Z. Wu, Z. Ye

The University of Iowa, Iowa City, U.S.A.

M. Alhusseini, K. Dilsiz ${ }^{91}$, R.P. Gandrajula, O.K. Köseyan, J.-P. Merlo, A. Mestvirishvili ${ }^{92}$, J. Nachtman, H. Ogul ${ }^{93}$, Y. Onel, A. Penzo, C. Snyder, E. Tiras ${ }^{94}$

Johns Hopkins University, Baltimore, U.S.A.

O. Amram, B. Blumenfeld, L. Corcodilos, J. Davis, M. Eminizer, A.V. Gritsan, S. Kyriacou, P. Maksimovic, J. Roskes, M. Swartz, T.Á. Vámi

The University of Kansas, Lawrence, U.S.A.

A. Abreu, J. Anguiano, C. Baldenegro Barrera, P. Baringer, A. Bean, A. Bylinkin, Z. Flowers, T. Isidori, S. Khalil, J. King, G. Krintiras, A. Kropivnitskaya, M. Lazarovits, C. Lindsey, J. Marquez, N. Minafra, M. Murray, M. Nickel, C. Rogan, C. Royon, R. Salvatico, S. Sanders, E. Schmitz, C. Smith, J.D. Tapia Takaki, Q. Wang, Z. Warner, J. Williams, G. Wilson

Kansas State University, Manhattan, U.S.A.

S. Duric, A. Ivanov, K. Kaadze, D. Kim, Y. Maravin, T. Mitchell, A. Modak, K. Nam

Lawrence Livermore National Laboratory, Livermore, U.S.A.

F. Rebassoo, D. Wright

University of Maryland, College Park, U.S.A.

E. Adams, A. Baden, O. Baron, A. Belloni, S.C. Eno, N.J. Hadley, S. Jabeen, R.G. Kellogg, T. Koeth, A.C. Mignerey, S. Nabili, C. Palmer, M. Seidel, A. Skuja, L. Wang, K. Wong

Massachusetts Institute of Technology, Cambridge, U.S.A.

D. Abercrombie, G. Andreassi, R. Bi, S. Brandt, W. Busza, I.A. Cali, Y. Chen, M. D'Alfonso, J. Eysermans, C. Freer, G. Gomez Ceballos, M. Goncharov, P. Harris, M. Hu, M. Klute, D. Kovalskyi, J. Krupa, Y.-J. Lee, C. Mironov, C. Paus, D. Rankin, C. Roland, G. Roland, Z. Shi, G.S.F. Stephans, J. Wang, Z. Wang, B. Wyslouch

University of Minnesota, Minneapolis, U.S.A.

R.M. Chatterjee, A. Evans, P. Hansen, J. Hiltbrand, Sh. Jain, M. Krohn, Y. Kubota, J. Mans, M. Revering, R. Rusack, R. Saradhy, N. Schroeder, N. Strobbe, M.A. Wadud 
University of Nebraska-Lincoln, Lincoln, U.S.A.

K. Bloom, M. Bryson, S. Chauhan, D.R. Claes, C. Fangmeier, L. Finco, F. Golf, C. Joo, I. Kravchenko, M. Musich, I. Reed, J.E. Siado, G.R. Snow ${ }^{\dagger}$, W. Tabb, F. Yan, A.G. Zecchinelli

State University of New York at Buffalo, Buffalo, U.S.A.

G. Agarwal, H. Bandyopadhyay, L. Hay, I. Iashvili, A. Kharchilava, C. McLean, D. Nguyen, J. Pekkanen, S. Rappoccio, A. Williams

Northeastern University, Boston, U.S.A.

G. Alverson, E. Barberis, Y. Haddad, A. Hortiangtham, J. Li, G. Madigan, B. Marzocchi, D.M. Morse, V. Nguyen, T. Orimoto, A. Parker, L. Skinnari, A. Tishelman-Charny, T. Wamorkar, B. Wang, A. Wisecarver, D. Wood

Northwestern University, Evanston, U.S.A.

S. Bhattacharya, J. Bueghly, Z. Chen, A. Gilbert, T. Gunter, K.A. Hahn, Y. Liu, N. Odell, M.H. Schmitt, M. Velasco

University of Notre Dame, Notre Dame, U.S.A.

R. Band, R. Bucci, A. Das, N. Dev, R. Goldouzian, M. Hildreth, K. Hurtado Anampa, C. Jessop, K. Lannon, J. Lawrence, N. Loukas, D. Lutton, N. Marinelli, I. Mcalister, T. McCauley, C. Mcgrady, K. Mohrman, Y. Musienko ${ }^{49}$, R. Ruchti, P. Siddireddy, A. Townsend, M. Wayne, A. Wightman, M. Zarucki, L. Zygala

The Ohio State University, Columbus, U.S.A.

B. Bylsma, B. Cardwell, L.S. Durkin, B. Francis, C. Hill, M. Nunez Ornelas, K. Wei, B.L. Winer, B.R. Yates

Princeton University, Princeton, U.S.A.

F.M. Addesa, B. Bonham, P. Das, G. Dezoort, P. Elmer, A. Frankenthal, B. Greenberg, N. Haubrich, S. Higginbotham, A. Kalogeropoulos, G. Kopp, S. Kwan, D. Lange, D. Marlow, K. Mei, I. Ojalvo, J. Olsen, D. Stickland, C. Tully

University of Puerto Rico, Mayaguez, U.S.A.

S. Malik, S. Norberg

Purdue University, West Lafayette, U.S.A.

A.S. Bakshi, V.E. Barnes, R. Chawla, S. Das, L. Gutay, M. Jones, A.W. Jung, S. Karmarkar, D. Kondratyev, M. Liu, G. Negro, N. Neumeister, G. Paspalaki, S. Piperov, A. Purohit, J.F. Schulte, M. Stojanovic ${ }^{16}$, J. Thieman, F. Wang, R. Xiao, W. Xie

Purdue University Northwest, Hammond, U.S.A.

J. Dolen, N. Parashar

Rice University, Houston, U.S.A.

A. Baty, M. Decaro, S. Dildick, K.M. Ecklund, S. Freed, P. Gardner, F.J.M. Geurts, A. Kumar, W. Li, B.P. Padley, R. Redjimi, W. Shi, A.G. Stahl Leiton, S. Yang, L. Zhang, Y. Zhang 
University of Rochester, Rochester, U.S.A.

A. Bodek, P. de Barbaro, R. Demina, J.L. Dulemba, C. Fallon, T. Ferbel, M. Galanti, A. Garcia-Bellido, O. Hindrichs, A. Khukhunaishvili, E. Ranken, R. Taus

\section{Rutgers, The State University of New Jersey, Piscataway, U.S.A.}

B. Chiarito, J.P. Chou, A. Gandrakota, Y. Gershtein, E. Halkiadakis, A. Hart, M. Heindl, O. Karacheban ${ }^{23}$, I. Laflotte, A. Lath, R. Montalvo, K. Nash, M. Osherson, S. Salur, S. Schnetzer, S. Somalwar, R. Stone, S.A. Thayil, S. Thomas, H. Wang

University of Tennessee, Knoxville, U.S.A.

H. Acharya, A.G. Delannoy, S. Fiorendi, S. Spanier

Texas A\&M University, College Station, U.S.A.

O. Bouhali ${ }^{95}$, M. Dalchenko, A. Delgado, R. Eusebi, J. Gilmore, T. Huang, T. Kamon ${ }^{96}$, H. Kim, S. Luo, S. Malhotra, R. Mueller, D. Overton, D. Rathjens, A. Safonov

Texas Tech University, Lubbock, U.S.A.

N. Akchurin, J. Damgov, V. Hegde, S. Kunori, K. Lamichhane, S.W. Lee, T. Mengke, S. Muthumuni, T. Peltola, I. Volobouev, Z. Wang, A. Whitbeck

Vanderbilt University, Nashville, U.S.A.

E. Appelt, S. Greene, A. Gurrola, W. Johns, A. Melo, H. Ni, K. Padeken, F. Romeo, P. Sheldon, S. Tuo, J. Velkovska

University of Virginia, Charlottesville, U.S.A.

M.W. Arenton, B. Cox, G. Cummings, J. Hakala, R. Hirosky, M. Joyce, A. Ledovskoy, A. Li, C. Neu, C.E. Perez Lara, B. Tannenwald, S. White, E. Wolfe

Wayne State University, Detroit, U.S.A.

N. Poudyal

University of Wisconsin - Madison, Madison, WI, U.S.A.

K. Black, T. Bose, C. Caillol, S. Dasu, I. De Bruyn, P. Everaerts, F. Fienga, C. Galloni, H. He, M. Herndon, A. Hervé, U. Hussain, A. Lanaro, A. Loeliger, R. Loveless, J. Madhusudanan Sreekala, A. Mallampalli, A. Mohammadi, D. Pinna, A. Savin, V. Shang, V. Sharma, W.H. Smith, D. Teague, S. Trembath-Reichert, W. Vetens

$\dagger$ : Deceased

1: Also at TU Wien, Wien, Austria

2: Also at Institute of Basic and Applied Sciences, Faculty of Engineering, Arab Academy for Science, Technology and Maritime Transport, Alexandria, Egypt, Alexandria, Egypt

3: Also at Université Libre de Bruxelles, Bruxelles, Belgium

4: Also at Universidade Estadual de Campinas, Campinas, Brazil

5: Also at Federal University of Rio Grande do Sul, Porto Alegre, Brazil

6: Also at The University of the State of Amazonas, Manaus, Brazil

7: Also at University of Chinese Academy of Sciences, Beijing, China

8: Also at Department of Physics, Tsinghua University, Beijing, China, Beijing, China

9: Also at UFMS, Nova Andradina, Brazil 
10: Also at Nanjing Normal University Department of Physics, Nanjing, China

11: Now at The University of Iowa, Iowa City, U.S.A.

12: Also at Institute for Theoretical and Experimental Physics named by A.I. Alikhanov of NRC 'Kurchatov Institute', Moscow, Russia

13: Also at Joint Institute for Nuclear Research, Dubna, Russia

14: Now at British University in Egypt, Cairo, Egypt

15: Also at Zewail City of Science and Technology, Zewail, Egypt

16: Also at Purdue University, West Lafayette, U.S.A.

17: Also at Université de Haute Alsace, Mulhouse, France

18: Also at Erzincan Binali Yildirim University, Erzincan, Turkey

19: Also at CERN, European Organization for Nuclear Research, Geneva, Switzerland

20: Also at RWTH Aachen University, III. Physikalisches Institut A, Aachen, Germany

21: Also at University of Hamburg, Hamburg, Germany

22: Also at Department of Physics, Isfahan University of Technology, Isfahan, Iran, Isfahan, Iran

23: Also at Brandenburg University of Technology, Cottbus, Germany

24: Also at Forschungszentrum Jülich, Juelich, Germany

25: Also at Physics Department, Faculty of Science, Assiut University, Assiut, Egypt

26: Also at Karoly Robert Campus, MATE Institute of Technology, Gyongyos, Hungary

27: Also at Institute of Physics, University of Debrecen, Debrecen, Hungary, Debrecen, Hungary

28: Also at Institute of Nuclear Research ATOMKI, Debrecen, Hungary

29: Also at MTA-ELTE Lendület CMS Particle and Nuclear Physics Group, Eötvös Loránd University, Budapest, Hungary, Budapest, Hungary

30: Also at Wigner Research Centre for Physics, Budapest, Hungary

31: Also at IIT Bhubaneswar, Bhubaneswar, India, Bhubaneswar, India

32: Also at Institute of Physics, Bhubaneswar, India

33: Also at G.H.G. Khalsa College, Punjab, India

34: Also at Shoolini University, Solan, India

35: Also at University of Hyderabad, Hyderabad, India

36: Also at University of Visva-Bharati, Santiniketan, India

37: Also at Indian Institute of Technology (IIT), Mumbai, India

38: Also at Deutsches Elektronen-Synchrotron, Hamburg, Germany

39: Also at Sharif University of Technology, Tehran, Iran

40: Also at Department of Physics, University of Science and Technology of Mazandaran, Behshahr, Iran

41: Now at INFN Sezione di Bari ${ }^{a}$, Università di Bari ${ }^{b}$, Politecnico di Bari ${ }^{c}$, Bari, Italy

42: Also at Italian National Agency for New Technologies, Energy and Sustainable Economic Development, Bologna, Italy

43: Also at Centro Siciliano di Fisica Nucleare e di Struttura Della Materia, Catania, Italy

44: Also at Università di Napoli 'Federico II', NAPOLI, Italy

45: Also at Consiglio Nazionale delle Ricerche - Istituto Officina dei Materiali, PERUGIA, Italy

46: Also at Riga Technical University, Riga, Latvia, Riga, Latvia

47: Also at Consejo Nacional de Ciencia y Tecnología, Mexico City, Mexico

48: Also at IRFU, CEA, Université Paris-Saclay, Gif-sur-Yvette, France

49: Also at Institute for Nuclear Research, Moscow, Russia

50: Now at National Research Nuclear University 'Moscow Engineering Physics Institute' (MEPhI), Moscow, Russia

51: Also at Institute of Nuclear Physics of the Uzbekistan Academy of Sciences, Tashkent, Uzbekistan 
52: Also at St. Petersburg State Polytechnical University, St. Petersburg, Russia

53: Also at University of Florida, Gainesville, U.S.A.

54: Also at Imperial College, London, United Kingdom

55: Also at Moscow Institute of Physics and Technology, Moscow, Russia, Moscow, Russia

56: Also at P.N. Lebedev Physical Institute, Moscow, Russia

57: Also at California Institute of Technology, Pasadena, U.S.A.

58: Also at Budker Institute of Nuclear Physics, Novosibirsk, Russia

59: Also at Faculty of Physics, University of Belgrade, Belgrade, Serbia

60: Also at Trincomalee Campus, Eastern University, Sri Lanka, Nilaveli, Sri Lanka

61: Also at INFN Sezione di Pavia ${ }^{a}$, Università di Pavia ${ }^{b}$, Pavia, Italy, Pavia, Italy

62: Also at National and Kapodistrian University of Athens, Athens, Greece

63: Also at Ecole Polytechnique Fédérale Lausanne, Lausanne, Switzerland

64: Also at Universität Zürich, Zurich, Switzerland

65: Also at Stefan Meyer Institute for Subatomic Physics, Vienna, Austria, Vienna, Austria

66: Also at Laboratoire d'Annecy-le-Vieux de Physique des Particules, IN2P3-CNRS, Annecyle-Vieux, France

67: Also at Şrnak University, Sirnak, Turkey

68: Also at Near East University, Research Center of Experimental Health Science, Nicosia, Turkey

69: Also at Konya Technical University, Konya, Turkey

70: Also at Piri Reis University, Istanbul, Turkey

71: Also at Adiyaman University, Adiyaman, Turkey

72: Also at Ozyegin University, Istanbul, Turkey

73: Also at Izmir Institute of Technology, Izmir, Turkey

74: Also at Necmettin Erbakan University, Konya, Turkey

75: Also at Bozok Universitetesi Rektörlügü, Yozgat, Turkey, Yozgat, Turkey

76: Also at Marmara University, Istanbul, Turkey

77: Also at Milli Savunma University, Istanbul, Turkey

78: Also at Kafkas University, Kars, Turkey

79: Also at Istanbul Bilgi University, Istanbul, Turkey

80: Also at Hacettepe University, Ankara, Turkey

81: Also at Istanbul University - Cerrahpasa, Faculty of Engineering, Istanbul, Turkey

82: Also at Vrije Universiteit Brussel, Brussel, Belgium

83: Also at School of Physics and Astronomy, University of Southampton, Southampton, United Kingdom

84: Also at Rutherford Appleton Laboratory, Didcot, United Kingdom

85: Also at IPPP Durham University, Durham, United Kingdom

86: Also at Monash University, Faculty of Science, Clayton, Australia

87: Also at Università di Torino, TORINO, Italy

88: Also at Bethel University, St. Paul, Minneapolis, U.S.A., St. Paul, U.S.A.

89: Also at Karamanoğlu Mehmetbey University, Karaman, Turkey

90: Also at Ain Shams University, Cairo, Egypt

91: Also at Bingol University, Bingol, Turkey

92: Also at Georgian Technical University, Tbilisi, Georgia

93: Also at Sinop University, Sinop, Turkey

94: Also at Erciyes University, KAYSERI, Turkey

95: Also at Texas A\&M University at Qatar, Doha, Qatar

96: Also at Kyungpook National University, Daegu, Korea, Daegu, Korea 